\title{
Modelling the asymmetric wind of the luminous blue variable binary MWC $314^{\star}$
}

\author{
A. Lobel ${ }^{1}$, J. H. Groh ${ }^{2}$, C. Martayan ${ }^{3}$, Y. Frémat ${ }^{1}$, K. Torres Dozinel ${ }^{4}$, G. Raskin ${ }^{5}$, H. Van Winckel ${ }^{5}$, S. Prins ${ }^{5}$, \\ W. Pessemier ${ }^{5}$, C. Waelkens ${ }^{5}$, H. Hensberge ${ }^{1}$, L. Dumortier ${ }^{1}$, A. Jorissen ${ }^{6}$, S. Van Eck ${ }^{6}$, and H. Lehmann ${ }^{7}$ \\ 1 Royal Observatory of Belgium, Ringlaan 3, 1180 Brussels, Belgium \\ e-mail: alobel@sdf.org; Alex.Lobel@oma.be \\ 2 Geneva Observatory, Geneva University, Chemin des Maillettes 51, 1290 Sauverny, Switzerland \\ 3 European Southern Observatory, 3107 Alonso de Cordova, Vitacura, Santiago, Chile \\ ${ }^{4}$ University of São João Del Rei, CAP, 36420-000 Ouro Branco, MG, Brazil \\ 5 University of Leuven, Instituut voor Sterrenkunde, Celestijnenlaan 200 D, 3001 Heverlee, Belgium \\ ${ }^{6}$ Université Libre de Bruxelles, Boulevard du Triomphe, 1050 Brussels, Belgium \\ 7 Thüringer Landessternwarte, Sternwarte 5, 07778 Tautenburg, Germany
}

Received 20 September 2012 / Accepted 17 July 2013

\section{ABSTRACT}

\begin{abstract}
Aims. We present a spectroscopic analysis of MWC 314, a luminous blue variable (LBV) candidate with an extended bipolar nebula. The detailed spectroscopic variability is investigated to determine if MWC 314 is a massive binary system with a supersonically accelerating wind or a low-mass B[e] star. We compare the spectrum and spectral energy distribution to other LBVs (such as P Cyg) and find very similar physical wind properties, indicating strong kinship.

Methods. We combined long-term high-resolution optical spectroscopic monitoring and $V$-band photometric observations to determine the orbital elements and stellar parameters and to investigate the spectral variability with the orbital phases. We developed an advanced model of the large-scale wind-velocity and wind-density structure with 3-D radiative transfer calculations that fit the orbitally modulated P Cyg profile of He I $\lambda 5876$, showing outflow velocities above $1000 \mathrm{~km} \mathrm{~s}^{-1}$.

Results. We find that MWC 314 is a massive semi-detached binary system of $\simeq 1.22 \mathrm{AU}$, observed at an inclination angle of $i=72.8^{\circ}$ with an orbital period of $60.8 \mathrm{~d}$ and $e=0.23$. The primary star is a low- $v \sin i \mathrm{LBV}$ candidate of $m_{1}=39.6 M_{\odot}$ and $R_{1}=86.8 R_{\odot}$. The detailed radiative transfer fits show that the geometry of wind density is asymmetric around the primary star with increased wind density by a factor of 3.3, which leads the orbit of the primary. The variable orientation causes the orbital modulation that is observed in absorption portions of P Cyg wind lines. Wind accretion in the system produces a circumbinary disc.

Conclusions. MWC 314 is in a crucial evolutionary phase of close binary systems, when the massive primary star has its $\mathrm{H}$ envelope being stripped and is losing mass to a circumbinary disc. MWC 314 is a key system for studying the evolutionary consequences of these effects.
\end{abstract}

Key words. stars: emission-line, Be - binaries: spectroscopic - stars: massive - stars: winds, outflows - line: profiles radiative transfer

\section{Introduction}

There is currently little known about the wind physics of the most massive binary stars. Although massive binary systems have been investigated for decades, it is notoriously difficult to infer important wind properties, such as accurate mass-loss rates, wind driving, and wind structuring mechanisms from traditional spectroscopic and photometric observations. Ultraviolet spectroscopy is one of the main sources for information about massive star winds but is currently available in only a small number of operational space-based instruments. Optical and near-IR long-term spectroscopic monitoring with ground-based spectrographs is an important alternative. In recent years the quality and efficiency of these high-resolution spectroscopic observations have dramatically improved, offering new opportunities for investigating detailed spectral line variability in the most massive binaries (e.g., Richardson et al. 2011).

The star MWC 314 (V1492 Aql; BD+14³887; $V=9^{\mathrm{m}} \cdot 9$ ) is a candidate luminous blue variable (LBV) and has been

* Table 1, Figs. 1-4, 9, 10, 14-16, 18-20, 23 are available in electronic form at http://www . aanda.org proposed as one of the most luminous stars in the Galaxy by Miroshnichenko et al. (1998) with $\log \left(L_{\star} / L_{\odot}\right) \simeq 6.1 \pm 0.3$, $T_{\text {eff }} \simeq 20$ to $30 \mathrm{kK}$, and $\dot{M} \sim 3 \times 10^{-5} M_{\odot} \mathrm{yr}^{-1}$ (assuming a single star). More recently, Muratorio et al. (2008) have found that the star is a (possible) binary system with an orbital period of $\sim 30 \mathrm{~d}$, however, without determining other orbital parameters. Marston \& McCollum (2008) demonstrated the presence of an extended double-lobe $\mathrm{H} \alpha$-emission (bipolar) nebula or a large east-west bipolar feature $>13$ pc (end to end) around the star. They suggest that the large size corresponds to a nebula age $\left(>10^{5} \mathrm{yr}\right)$ that is greater than for LBV nebula in general. The lifetime of the LBV phase is, however, quite uncertain and may be much longer $\left(2-5 \times 10^{5} \mathrm{yr}\right.$; Humphreys \& Davidson 1994) than previously assumed. The LBV phase can be the evolutionary phase, when most of the mass loss of the most massive stars occurs to form the enigmatic Wolf-Rayet stars. In addition, recent evolutionary and radiative transfer calculations have shown that low-luminosity LBVs can be the end stage of massive star evolution (Groh et al. 2013).

A persisting problem for analysing and interpreting the spectrum of MWC 314 is that only a small number of stellar 
absorption features have been observed and identified thus far. The optical spectrum is, however, dominated by strong and single-peaked $\mathrm{H}$ Balmer emission and $\mathrm{He}$ I lines. Numerous metallic Fe II, [Fe II], Cr II, and Ti II emission lines were identified by Swensson (1943). The double-peaked shape of the metallic emission lines signal formation in a rotating disc and could indicate a $\mathrm{B}[\mathrm{e}]$ star in lieu of an LBV. Muratorio et al. (2008) therefore conjectured that the disc is produced by lowvelocity outflow, as in $\mathrm{B}[\mathrm{e}]$ supergiants, instead of an accretion mechanism, although they do not rule out accretion disc formation. Except for the $\mathrm{H} \alpha$ equivalent width variability observed by Wisniewski et al. (2006), there is no evidence in the literature of significant spectral line variability that can confirm an LBV nature for MWC 314.

P Cygni-type optical line profiles due to expanding wind opacity have not been observed thus far around MWC 314 (see also Sect. 6.2 of Marston \& McCollum 2008). In this paper, we show that the optical He I lines of MWC 314 are P Cyg profiles, with detailed shape variability that correlates to the orbital phases. We show that MWC 314 is spectroscopically, and in its overall spectral energy distribution (SED), almost identical to the LBV P Cygni. The bipolar nebula observed around MWC 314, combined with its LBV-like spectroscopic properties, signal a unique massive binary system that can possibly contain an LBV in a dormant state. The candidate LBVs MWC 349A and Sher 25 have comparable physical properties. MWC 349A was recently proposed to be an LBV by Gvaramadze et al. (2010) and Sher 25 earlier by Brandner et al. (1997). Interestingly, six of the nine known Galactic LBVs are also surrounded by bipolar nebulae.

There are currently two proposed LBV binaries, $\eta$ Car (Damineli et al. 2008) and HD 5980. The binary system $\eta$ Car is well-studied, currently involving sophisticated hydrodynamic wind modelling efforts by Madura et al. (2012) and Okazaki et al. (2008). HD 5980 is an eclipsing OB+WR binary system (Hoffman et al. 1978) with an orbital period of $\simeq 19.3 \mathrm{~d}$. More recently, it has been identified as an LBV/WR binary (containing a third star) with stellar and orbital parameters that have been relatively well determined by Georgiev et al. (2011) and Foellmi et al. (2008). The third (and possibly fourth) components appear to be a pair of fairly normal, understudied O-type stars either coincident to the line of sight or in a very long orbit with HD 5980AB. MWC 314 is also very similar to massive binary HDE 269128 (RMC 81) in the LMC with $P=74.655 \mathrm{~d}$ (Tubbesing et al. 2002). RMC 81 is an eclipsing close binary $(e=0.57)$ with an cLBV primary star of $T_{\text {eff }}=19500 \mathrm{~K}$, $R_{\star}=96 R_{\odot}$, and $K_{1}=35 \mathrm{~km} \mathrm{~s}^{-1}$.

More recently, Martayan et al. (2012) have imaged a companion near the cLBV HD 168625 for the first time with Very Large Telescope/NACO (Nasmyth Adaptive Optics System). The system is known to be surrounded by several rings similar to those of SN1987A. We note, however, that the high $V$-brightness changes typical of bona fide LBVs, presumably due to recurrent eruption activity, have so far not been observed in MWC 314. It is therefore technically considered a cLBV without signs of S Dor variability as for AG Car and HR Car. We find in this paper that the slow rotation of MWC 314 fits well in the scheme in which fast-rotating LBVs show S Dor variability, while slowly rotating ones do not (Groh et al. 2009a).

We spectroscopically monitored MWC 314 for two years to test for binarity (Lobel et al. 2012). We present the observations in Sect. 2. In Sect. 3, we discuss new $\mathrm{H} \alpha$ imaging observations of inner envelope emission in MWC 314. Section 4 presents the high-resolution optical and near-IR spectra with radial velocity measurements for determining the binary period and other orbital elements. The spectra are combined with an analysis of ASAS $V$-band photometry that also provides accurate stellar parameters. Detailed spectroscopic analysis reveals strong correlations and clear dependence of permitted and forbidden emission line variability on the orbital phases, which we observe, for example, in variable $\mathrm{H} \alpha, \mathrm{H} \beta$, and metallic emission lines. We also present a detailed comparison to the SED of P Cygni using broadband photometry combined with IR Spitzer and ISO spectra. The IR SEDs of MWC 314 and P Cyg are practically identical. In Sect. 5, we present a quantitative model of the large-scale asymmetric wind structure around MWC 314 that is computed with 3D radiative transfer by modelling the orbitally modulated P Cyg line profile variability in detail. We discuss and summarise the results in Sect. 6. We show that MWC 314 is a massive binary system and accurately determine its orbital and stellar parameters. We find that MWC 314 can contain an LBV star with wind properties comparable to $\mathrm{P}$ Cygni and put strong constraints on the stellar masses and the size of the system.

\section{Observations}

\section{1. Échelle spectroscopy}

We observed 15 high-resolution spectra $(R \simeq 85000)$ of MWC 314 between Sep 2009 and Aug 2011 with the HERMES instrument of the 1.2-m Mercator Telescope at the Roque de los Muchachos Observatory on La Palma Island (Canary Islands, Spain). HERMES is a high-efficiency bench-mounted échelle spectrograph that observes the complete wavelength range from $420 \mathrm{~nm}$ to $900 \mathrm{~nm}$ in a single exposure (Raskin et al. 2011). The HERMES spectra of MWC 314 have large signal-tonoise ratio $(\mathrm{S} / \mathrm{N}) \sim 100$ required for accurate radial velocity measurements and detailed studies of line profile variability. Three subsequent exposures of $\sim 1800 \mathrm{~s}$ each are added to minimise the amount of CCD cosmic hits. The spectra are calibrated with the latest version of the HERMES pipeline (release 4.0) developed at the Royal Observatory of Belgium (ROB) in collaboration with the HERMES Consortium. The typical échelle calibration steps are performed, which include spectral order tracing and extraction, average flat-fielding, Th-Ar lamp wavelength calibration, and hot pixel removal using cross-order profiling. The wavelength scale is corrected to the heliocentric rest frame, which for most observations includes a wavelength rebinning of the sum spectra. An overview of the HERMES observations of MWC 314 is provided in Table 1. On 5 and 10 Sep. 2009 and on 18 and 21 Mar 2011, we observe two spectra within $5 \mathrm{~d}$ to investigate short-period spectroscopic variability in MWC 314.

One FEROS spectrum of MWC 314 is retrieved from the ESO Science Archive Facility. The FEROS spectrum of 5 June 2009 precedes the start of our HERMES monitoring campaign by only $\sim 3 \mathrm{~m}$ and is very useful for testing the orbital solution for MWC 314 that we discuss in Sect. 4.1. The FEROS instrument on the MPG/ESO 2.2-m telescope is a bench-mounted fiber-linked high-resolution $(R=48000)$ échelle spectrograph with high efficiency ( $20 \%$ between $5000 \AA$ and $6000 \AA)$, covering the wavelength range from $356 \mathrm{~nm}$ to $921 \mathrm{~nm}$ with 39 orders in one exposure. For example, a $V=12$. 0 star can be observed with a S/N of 105 in two hours (Kaufer et al. 1999). The spectrum of MWC 314, observed for $900 \mathrm{~s}$, is calibrated in ESO-MIDAS with the FEROS-data reduction system (DRS). The common échelle pipeline reduction steps are performed starting with bias subtraction, optimal order extraction, and division of the science exposures with an averaged flat-field frame, which is 
followed by the order wavelength calibration using a Th-Ar lamp spectrum. The flat-fielding step in the FEROS-DRS pipeline removes the blaze-function similar to the HERMES calibration pipeline.

\subsection{Imaging and photometry}

We observed MWC 314 on 10 Mar 2011 with the MEROPE instrument on Mercator: MEROPE is a direct camera in the Cassegrain focal station of the telescope. The camera chip is a $2 \mathrm{k} \times 2 \mathrm{k}$ back-side illuminated device cooled with liquid nitrogen to an operational temperature of $-130{ }^{\circ} \mathrm{C}$. The total field of view is $6.6^{\prime} \times 6.6^{\prime}$ (standard setting) with a sampling of $0.193^{\prime \prime}$ per pixel (Le Guillou et al. 2008). The inner portions of the extended bipolar $\mathrm{H} \alpha$ nebula of MWC 314 were observed with three subsequent MEROPE exposures. The star was first observed during $300 \mathrm{~s}$ through a special narrow-band $\mathrm{H} \alpha$ filter. The filter's transmission function has a full width at half maximum (FWHM) $\sim 50 \AA$ with a peak transmittance of $\sim 90 \%$ at $\mathrm{H} \alpha$. The passband is very narrow with transmittance levels, decreasing below $10 \%$ shortward of $6520 \AA$ and longward of $6610 \AA$. Next, a broad passband optical continuum filter, which excludes $\mathrm{H} \alpha$, is used for comparison during $200 \mathrm{~s}$. The continuum filter has a transmission function $F W H M \sim 100 \AA$ centred around $6365 \AA$.

Finally, a second $\mathrm{H} \alpha$ image is observed for $414 \mathrm{~s}$ to increase the combined $\mathrm{S} / \mathrm{N}$. Master flat-field images are observed in both filters before and after the target sequence. The image calibration steps include bias level measurement of the mean value in the overscan areas. The mean bias level is subtracted from the combined flat-field frames and the science exposures before flat division. The comparison of the $\mathrm{H} \alpha$ and continuum MEROPE images of MWC 314 is discussed in Sect. 3. It also provides a comparison of the inner $\mathrm{H} \alpha$ nebula surrounding MWC 314 and its extended bipolar nebula observed by Marston \& McCollum (2008).

We retrieved photometric fluxes of MWC 314 in the $V$-band from the All Sky Automated Survey (ASAS) project public database (Pojmanski 2002); ASAS-3 observations of MWC 314 were performed at Las Campanas Observatory (Chile) between Mar. 2003 and Oct. 2009. The entire facility is housed in an automated enclosure and consists of four telescopes, equipped with a telelens and a $2 \mathrm{k} \times 2 \mathrm{k}$ CCD camera that observes wide and narrow fields of the sky through $V$ - and $I$-band filters. The exposure time is $180 \mathrm{~s}$ per frame and $372 \mathrm{~V}$-band observations (quality grades A to D) are offered of which we select 280 with the highest quality (grade A only). The typical $V$-error is $0.03-0.04$. It is of note that the FEROS spectrum of June 2009 and the HERMES observations of Sep. 2009 are coeval with the ASAS-3 observations of MWC 314. The comparison of the radial velocity values (RV) and the corresponding $V$-magnitudes rules out the $\sim 1 \mathrm{~m}$ orbital period proposed in Muratorio et al. (2008). The $V$-brightness curve consists of two minima over a variability period of $\sim 2 \mathrm{~m}$. In Sect. 4.2, we further discuss the ASAS $V$-curve in relation to the RV monitoring of MWC 314.

\section{3. $\mathrm{H} \alpha$ imaging}

Figure 1 shows the MEROPE narrow band $\mathrm{H} \alpha$ image that we observed around MWC 314 (inset image). The outer flux image is adapted from Fig. 1 of Marston \& McCollum (2008). The MEROPE image is contoured in the linear intensity scale to enhance the faint $\mathrm{H} \alpha$ nebulosity. The intensity contour image is very practical for cross-comparison with the continuum passband image in the right-hand panel of Fig. 2. The faint eastwest $\mathrm{H} \alpha$ emission is not detected in the broadband continuum image. With the absence of other similarly strong emission lines in the optical spectrum, we find that MWC 314's bipolar nebula primarily consists of an extended loop-like filament structure of ionised hydrogen gas. We observe very strong and broad $\mathrm{H} \alpha$ emission with line peak fluxes more than 20 times above the continuum flux level and equivalent line widths of 120-150 $\AA$ (see Sect. 4.4.2). The aperture size of our spectroscopic $\mathrm{H} \alpha$ observations is limited to $\simeq 2^{\prime \prime}$, and MEROPE imaging is therefore useful for detecting possible spatial asymmetry of $\mathrm{H} \alpha$ emission that is related to the extended E-W lobe structures.

We devised various image calibration methods but were unable to detect any $\mathrm{H} \alpha$ flux asymmetry within $20^{\prime \prime}$ from the central source. The left-hand panel of Fig. 3 shows a narrow-band $\mathrm{H} \alpha$ subimage of $526 \times 526$ pixels for a field of view of $100^{\prime \prime}$ by $100^{\prime \prime}$. The continuum subimage is shown in the right-hand panel. The angle between two tick marks is 100 pixels or $100 \times$ $0.19^{\prime \prime}=19^{\prime \prime}$. The inner (nearly Gaussian) intensity distribution is practically circular and symmetric in both contour images, having a HWHM of $7.0 \pm 0.5$ pixels or $7 \times 0.19^{\prime \prime}=1.33^{\prime \prime}$. The innermost contour is drawn at a level of $1.82 \%$ of the central intensity maximum. The outermost contour is drawn at $0.92 \%$ (dots). The comparison of these intensity levels clearly reveals the larger spatial extension of the $\mathrm{H} \alpha$ emission envelope around MWC 314. The contours of the stars towards the S and SW have the same (almost circular) shape in both images. Only at distances well beyond $20^{\prime \prime}$ from the central star, where $\mathrm{H} \alpha$ flux levels rapidly decreases to below $0.5 \%$ of the central peak intensity, do we observe patchy regions of faint $\mathrm{H} \alpha$ emission. Our imaging observations therefore do not indicate a preferred direction of (wind) outflow that may (have) produce(d) the extended bipolar $\mathrm{H} \alpha$ nebula. The images do reveal that the inner $\mathrm{H} \alpha$ envelope is directly linked to MWC 314 and associated with very bright $\mathrm{H} \alpha$ line emission emerging from the central binary system.

\section{Spectroscopic and photometric variability}

\subsection{Orbital elements and stellar parameters}

We investigate a number of unblended absorption lines for accurate RV measurements. Three lines S II $\lambda 5454.8, \lambda 5473.6$, and $\lambda 5647.0$, and one line $\mathrm{Ne}$ I $\lambda 6402.2$ are selected, because they show well-defined flux minima (for $S / N>100$ ) with respect to the neighbouring continuum flux level. We measure the RV of each line from the line bisector at half flux minimum after local continuum normalisation. The spectra are converted to the heliocentric rest frame, and we estimate the rms error of the $\mathrm{RV}$-values based on the noise levels of fluxes observed inside these lines. For the strongest line, the RV mean errors stay below $\pm 3-7 \mathrm{~km} \mathrm{~s}^{-1}$, which can nearly double for the weaker absorption lines.

We use the PHOEBE code of Prša \& Zwitter (2005) for computing the best fit to the RV-curve and the ASAS $V$-brightness curve provided in heliocentric Julian days (HJD). Figure 4 shows the $16 \mathrm{RV}$-values that we measure (see Table 1) from the spectra with the best fit solutions from PHOEBE (curved solid lines in top and bottom panels). PHOEBE calculates the best fit orbital period $(P)$ by varying the values of the eccentricity $(e)$, maximum distance between both stars $(a)$, systemic velocity $(\gamma)$, orbital inclination angle $(i)$, and longitude of periastron $(\omega)$. The best fit solution to the combined RV- and $V$-curve also varies the mass ratio of both stars. The code computes the stellar masses and radii with corresponding surface gravities. It also 


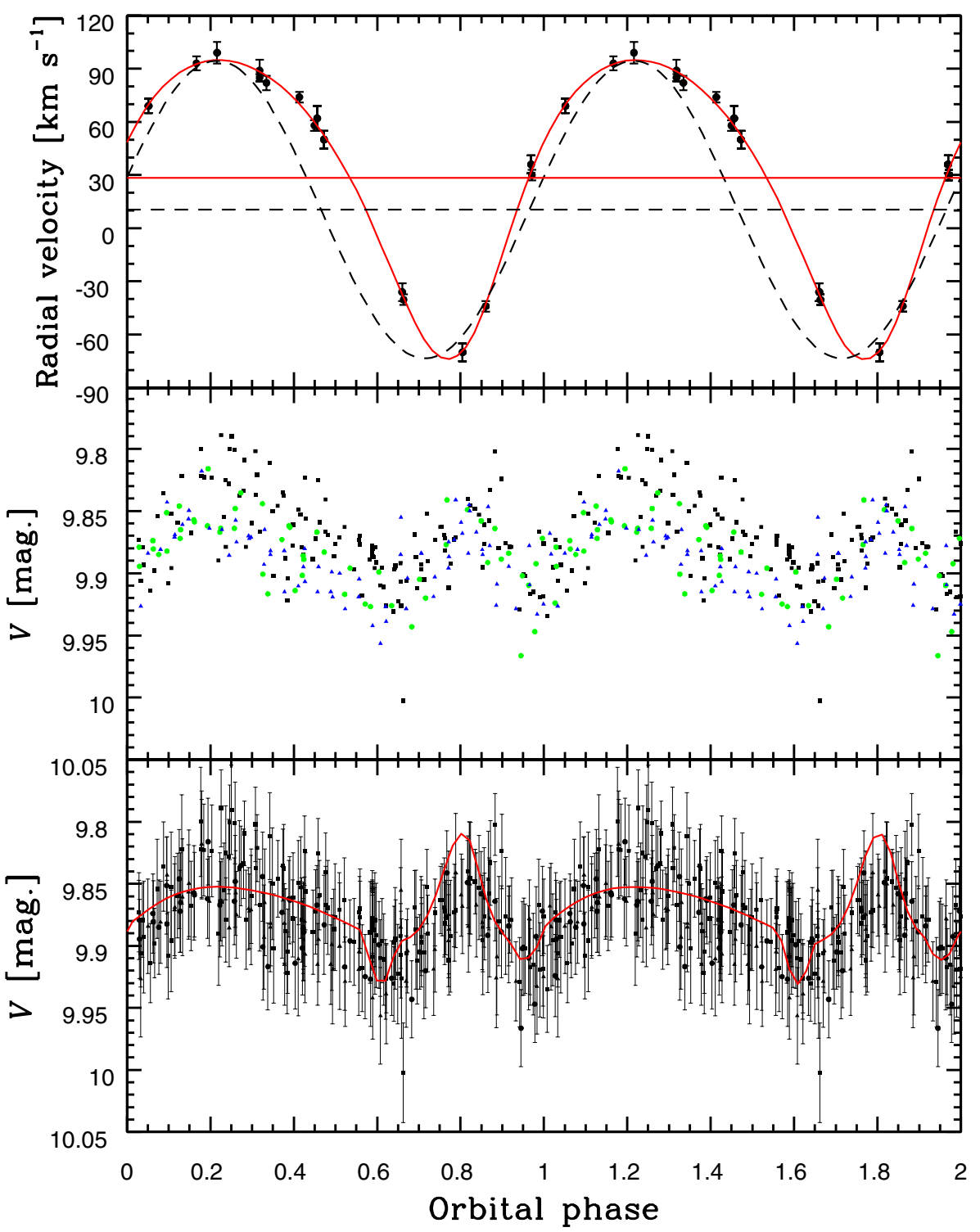

Fig. 5. Top panel: best fit radial velocity curve (curved solid line) as a function of orbital phase. The orbital radial velocity curve signals an eccentric orbit of $e=0.23$ and $P=60.8 \mathrm{~d}$. A circular orbit $(e=0)$ does not fit (curved dashed line) the observations (solid dots). The $\gamma$-velocity of $+28.4 \mathrm{~km} \mathrm{~s}^{-1}$ (solid horizontal line) is larger than $12.3 \mathrm{~km} \mathrm{~s}^{-1}$ (dashed horizontal line) when $e$ is set equal to 0 . Middle panel: the ASAS $V$-brightness curve shows two minima signaling an eclipsing binary system. Bottom panel: the symbols mark the $V$ brightness values in the middle panel with errorbars. The model best fit is drawn with a smooth curve (see Sect. 4.1). gives the surface brightness ratio, employing a logarithmic limb darkening law for both stars. We use the PHOEBE internal fitting method of differential corrections and obtain the lowest $\chi^{2}$-values of 0.01248 for the $280 V$-points and of 0.01180 for the $16 \mathrm{RV}$-points.

Table 2 lists the best fit orbital elements and stellar parameters of MWC 314. The error values of the orbital elements are computed from the errors of the RV and $V$ input data. The $\mathrm{RV}$ rms errors are listed in Table 1, while the $\sigma$-errors of $V$ stay typically below \pm 0 . 05 , as shown in the bottom panel of Fig. 5 . The top panel of Fig. 5 shows the best fit RV-curve as a function of orbital phase $(\phi)$. An eccentric orbit with $e=0.23$ best fits the RV observations, while a circular orbit $(e=0)$ does not. We find that the systemic velocity of $\simeq+28 \mathrm{~km} \mathrm{~s}^{-1}$ is indeed very close to the bisector positions of many static metal emission lines (see Sect. 4.4.3).

The ASAS $V$-curve covers $\sim 38$ orbital periods (or cycles). There is substantial scatter that can be due to a long-term trend observed towards fainter magnitudes in 2008-2009, when compared to 2003-2006. It can be due to multiple periodicity and/or cycle-to-cycle variations, since it substantially decreases when using only cycles 20 to 38 . This is even more apparent when using data from cycles 30 to 38 only. In the middle panel
Table 2. Orbital elements and stellar parameters of the stars in MWC 314 obtained from the simultaneous best fit to the RV curve and ASAS $V$-magnitude curve with the PHOEBE code; $a=a_{1}+a_{2}$ is the maximum distance between both stars.

\begin{tabular}{lc}
\hline \hline Element & Value \\
\hline$P[\mathrm{~d}]$ & $60.799977 \pm 0.000014$ \\
$T$ (HJD) & $2454959.76 \pm 0.56$ \\
$e$ & $0.235 \pm 0.003$ \\
$\omega[\mathrm{deg}]$ & $289 \pm 1$ \\
$\gamma\left[\mathrm{km} \mathrm{s}^{-1}\right]$ & $28.44 \pm 0.17$ \\
$a\left[R_{\odot}\right]$ & $262.58 \pm 19.52$ \\
$i[\mathrm{deg}]$ & $72.79 \pm 13.05$ \\
\hline Primary star & \\
$T_{\text {eff }}[\mathrm{K}]$ & 18000 \\
$\log g[\mathrm{dex}]$ & 2.26 \\
$m_{1}\left[M_{\odot}\right]$ & 39.66 \\
$R_{1}\left[R_{\odot}\right]$ & 86.80 \\
$K_{1}\left[\mathrm{~km} \mathrm{~s}{ }^{-1}\right]$ & 84.5 \\
\hline Secondary star & \\
$T_{\text {eff }}[\mathrm{K}]$ & 6227 \\
$\log g[\mathrm{dex}]$ & 3.55 \\
$m_{2}\left[M_{\odot}\right]$ & 26.26 \\
$R_{2}\left[R_{\odot}\right]$ & 20.41 \\
\hline
\end{tabular}



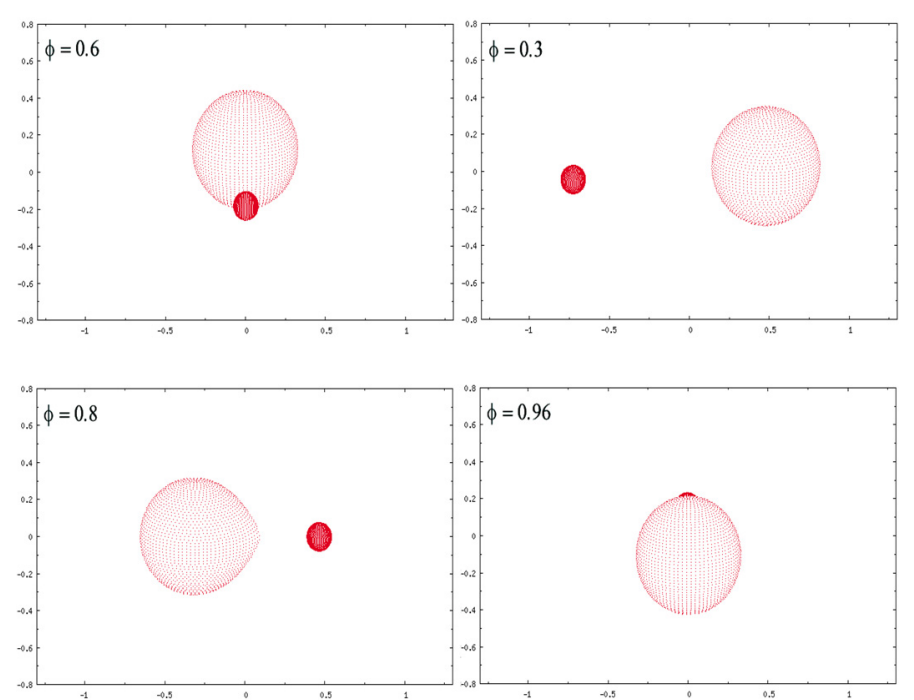

Fig. 6. Schematic representation of four orbital phases (counterclockwise) in MWC 314 computed with the PHOEBE code.

of Fig. 5, we fold the $V$ data with $P=60.8 \mathrm{~d}$. The scatter in $V$ over three time intervals is shown with three different symbols. The $V$-curve shows two brightness minima with the orbital phases: the main minimum is around $\phi=0.55-0.65$, and a shallower minimum is around $\phi=0.95-0.05$. The mean amplitude does not exceed $0 . \mathrm{m} 1$, but the cycle-to-cycle scatter between $V$-extrema is $\simeq 0$. 15 . The unequal brightness minima are due to an eclipsing binary system. The main minimum is due to partial eclipses by the companion star of the more luminous primary, while the companion is partly occulted by the primary star in the shallower $V$-minimum.

It is important to point out that the orbital and stellar parameters in Table 2 are computed with PHOEBE using $T_{\text {eff }}=18000 \mathrm{~K}$ for the primary star. We determine the latter value from detailed non-LTE fits to three Si III absorption lines in Sect. 4.2. By varying $T_{\mathrm{eff}}$ of the secondary star, PHOEBE computes the minimum $\chi^{2}$-values by simultaneously best fitting the RV- and $V$-observations. We obtain $T_{\text {eff }}=6227 \mathrm{~K}$ for the secondary star. It yields a surface brightness ratio of $\sim 15$. The slow $V$-brightness variability modelled with the code results from the deformation from spherical shape of the primary's surface in a semi-detached binary system. The primary fills its Roche lobe and alters the projected surface area (against the sky) during its orbit. Figure 6 shows the increase in projected surface area at $\phi=0.8$ around maximum $V$ brightness (bottom left-hand panel). The surface deformation is smaller at $\phi=0.3$ (top right-hand panel), because the distance between the stars further increases towards apastron around $\phi=0.6$ and the partial eclipse by the secondary star.

During periastron passage, the shape deformation of the primary is strongest but mostly behind the star for $i=72^{\circ}$, while the occultation of the secondary causes a stronger brightness decrease of $V$. We do not incorporate reflections or spots in the PHOEBE model of the $V$-curve. The best fit signals a yellow supergiant secondary star with a small radius. Interestingly, the binary system HDE 326823 also has a large mass-function with a secondary that is likely obscured by a thick torus (Richardson et al. 2011). The best $V$-fit with PHOEBE of MWC 314 is sensitive to $i$, and the brightness minima result from the partial eclipses. In the case there is a thick torus around the secondary, the torus' optical thickness in $V$ changes the duration of the brightness minima.

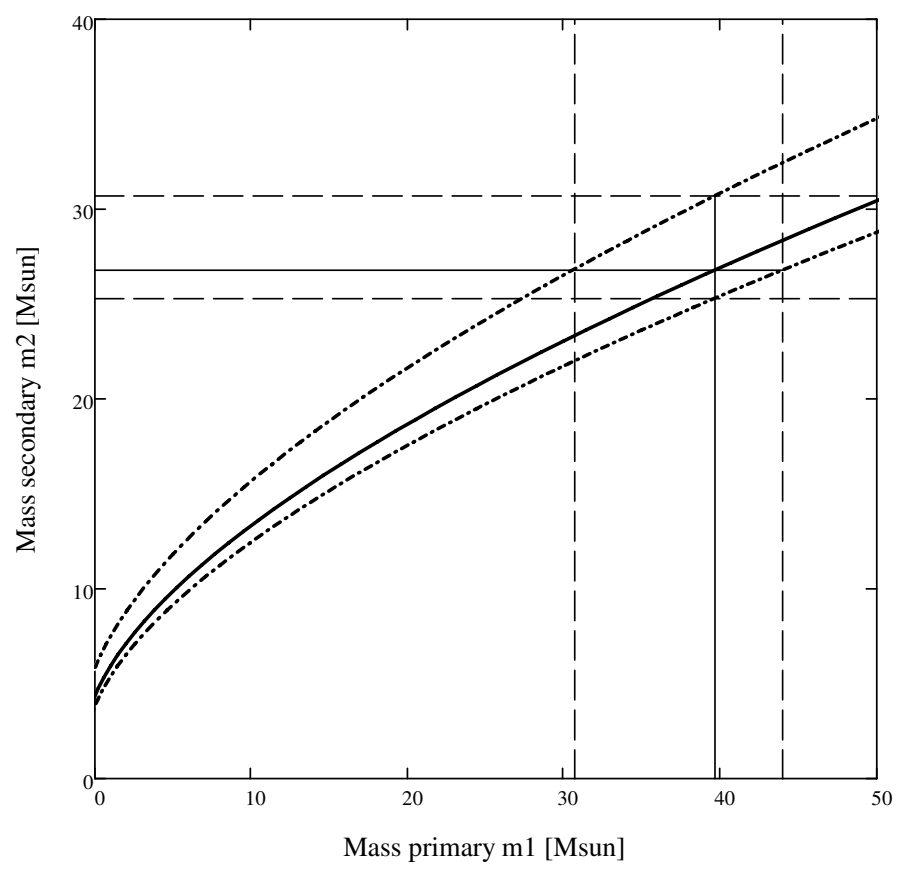

Fig. 7. Mass function of MWC 314 computed for $i=72.8^{\circ}$ (thick drawn solid line), which is also shown for inclination angles of $i-13^{\circ}$ (top dash-dotted line), and $i+13^{\circ}$ (bottom dash-dotted line). The solid drawn vertical and horizontal lines mark the values of $m_{1}=39.66 M_{\odot}$ and $m_{2}=26.26 M_{\odot}$ computed with PHOEBE, while the dashed drawn lines mark the boundaries of $30.7 M_{\odot} \leq m_{1} \leq 44.2 M_{\odot}$ and $25.3 M_{\odot} \leq$ $m_{2} \leq 30.7 M_{\odot}$ corresponding to inclination angles ranging from $i-13^{\circ}$ to $i+13^{\circ}$.

We compute an rms errorbar of $\pm 13^{\circ}$ to the best fit value of $i$ with PHOEBE. We use the error value for computing errors to the provided stellar masses. Figure 7 shows $m_{2}$ as a function of $m_{1}$ for $i=72.8^{\circ}$. We compute

$\frac{m_{2}^{3}}{m^{2}} \sin ^{3} i=\frac{P}{2 \pi G} K_{1}^{3}$,

where $m=m_{1}+m_{2}=65.92 \pm 9 M_{\odot}$ and the semi-amplitude $K_{1}$-velocity of $\simeq 84.5 \mathrm{~km} \mathrm{~s}^{-1}$ from the RV-curve. The solid drawn vertical and horizontal lines mark the best fit values of $m_{1}=$ 39.66 $M_{\odot}$ and $m_{2}=26.26 M_{\odot}$. The dashed drawn lines mark the computed error boundaries of $30.7 M_{\odot} \leq m_{1} \leq 44.2 M_{\odot}$ and $25.3 M_{\odot} \leq m_{2} \leq 30.7 M_{\odot}$ for $i \pm 13^{\circ}$. The mass we compute for MWC 314 signals a massive binary system of $m>65 M_{\odot}$ with stellar mass errors below $\pm 9 M_{\odot}$. The total bolometric luminosity $L_{\star}$ of the system is $7.1 \times 10^{5} L_{\odot}$. The latter value is lower than previous estimates (i.e., Miroshnichenko et al. 1998), but appears to be a normal trend for stars in the upper portion of the H-R diagram (for example also in the Pistol star). The $L_{\star}$ of MWC 314 is close to the Eddington luminosity limit with $\Gamma_{\text {Edd }}>0.5$ for electron scattering. It is much higher than in other B-type hypergiants (such as Cyg OB2 No. 12, BP Cru, and HD 152236, see Clark et al. 2012), placing MWC 314 in the ballpark of an LBV.

Figure 8 depicts the orbit of the primary star in MWC 314. After the main $V$-brightness minimum, the primary approaches the observer with the largest RV (e.g., maximum blueshift of the absorption lines) at around $\phi \simeq 0.75-0.8$. At the largest elongation, the total brightness is at a maximum. Since we compute $\omega=289^{\circ}$ (Table 2), periastron occurs around $\phi=0.12$. The dashed line in Fig. 8 is the primary orbit computed for $e=$ 0.23 around the centre-of-mass of the binary system. The orbital 


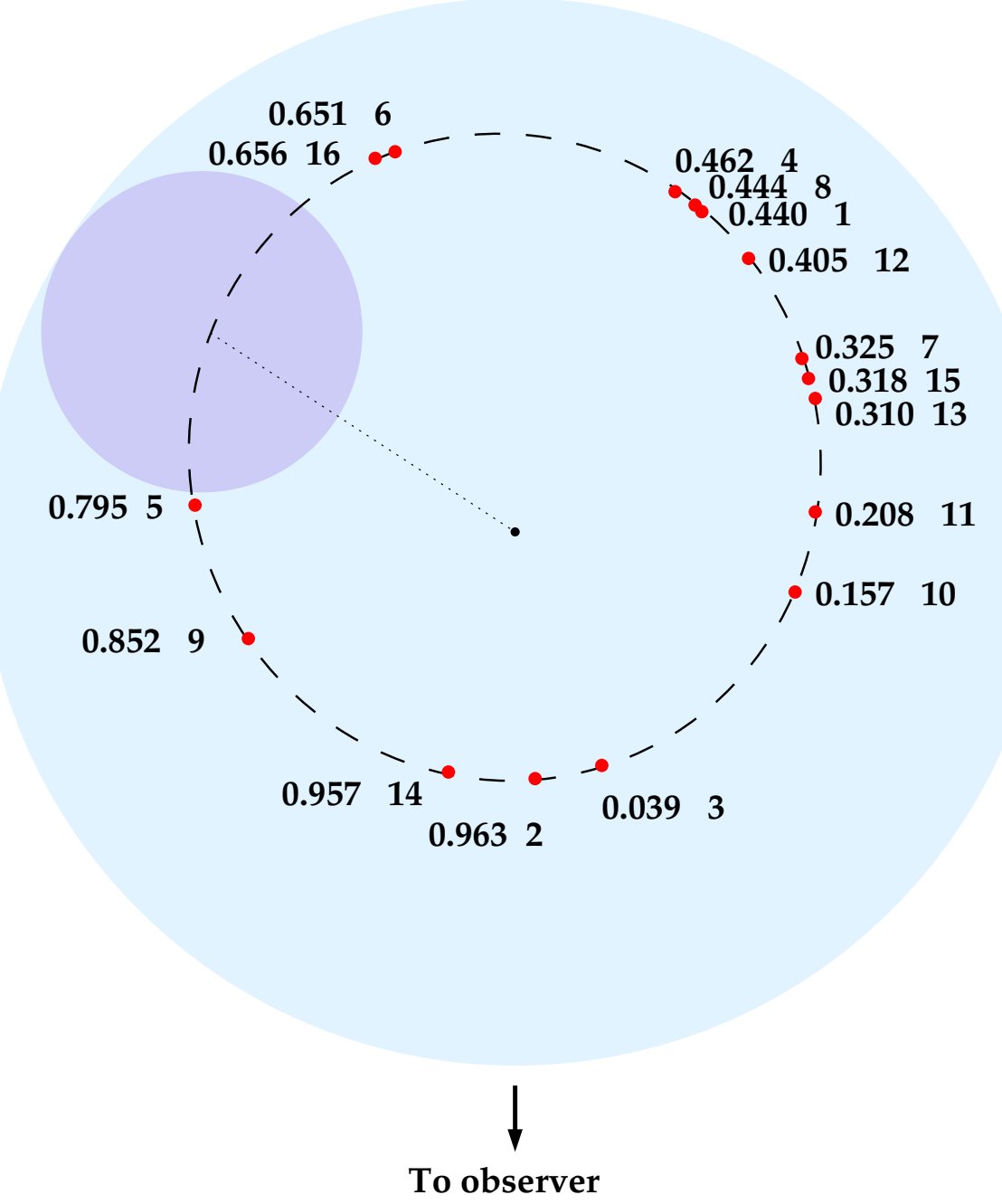

Fig. 8. Orbital path (dashed line) of the primary of MWC 314 shown around the centre of gravity of the binary (central black dot) and marked for the 16 observed orbital phases (solid dots). The labels provide the $\phi$-values with spectrum numbers in Table 1 . The shape and orientation of the orbit are set by $e$ and $\omega$. The disc to the left shows the orbital position of the primary star at $\phi \sim 0.7$. The large outer disc represents the circumbinary wind region extending further away from the gravity centre. phases that we observe are marked. We compute the semi-major axis of $a_{1} \sin i=98.7 \pm 6 R_{\odot}$ with $K_{1}$ and $P$. The periastron distance, computed with $a_{1}(1-e)$ and $i=72.8^{\circ} \pm 13^{\circ}$, is $79.5 \pm$ $8 R_{\odot}$. The apastron distance is $127.0 \pm 13 R_{\odot}$. The long axis of the primary's orbit is $79.5+127.0 R_{\odot}=206.5 R_{\odot}$, or very close to $1 \mathrm{AU}$.

\subsection{Absorption lines and atmospheric parameters}

We investigated the continuum normalised line profiles of three absorption lines of MWC 314 in Fig. 9. The bottom panels show flux plots evenly stacked according to the orbital phase to avoid overcrossing lines. The spectrum numbers are labelled in the bottom left-hand panel. The top panels show the corresponding dynamic spectra in the heliocentric velocity scale. We observe that the depth and width of the S II and Ne I absorption lines do not substantially vary over the orbit, although the lines become deeper $(\sim 15 \%)$ for $\phi=0.65-0.85$. We performed detailed comparisons of the absorption line profiles and do not find indications that these high-excitation lines are influenced by emission contributions with orbital phase. It signals that the RV shifts result from orbital motion of the entire absorption line formation region and that Doppler effects of possible atmospheric pulsations stay small. This is also supported by the high total RV amplitude of $169 \mathrm{~km} \mathrm{~s}^{-1}$, which with the short period of $P=60.8 \mathrm{~d}$, rules out RV variability caused by stellar pulsation.

Miroshnichenko et al. (1998) identify a good number of S II (3), N II (2), Ne I (4), and Al III (2) absorption lines in the optical spectrum of MWC 314 (see their Table 5). We search for other doubly ionised absorption lines, such as C III $\lambda 4647.42$ and $\lambda 4650.25$, but do not find any evidence of them. We neither detect the N III absorption lines at $\lambda 6478$ and $\lambda 6487$. We observe the Si III absorption line at $\lambda 5739.7$, and the Si III triplet lines at $\lambda 4552.62, \lambda 4567.82$, and $\lambda 4574.76$. The last line is a weak absorption line blended in the red wing due to veiling from neighbouring emission lines. We do not find any evidence

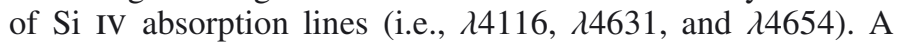
study of the Si II/Si III-ionisation balance in MWC 314 turns out to be complicated in that many of the Si II lines are filled in by (static) emission above the continuum flux level. The photospheric Si II absorption line becomes noticeable during some orbital phases (see Sect. 4.4.4 for a discussion of Si II 26347 ), but the emission contributions severely compromise the Si II lines for reliable depth and equivalent width (EW) measurements.

We performed detailed radiative transfer fits to three Si III lines using the MulTI code (Carlsson 1986) for determining atmospheric parameters of the primary. The $\lambda 4552.62$, $\lambda 4567.82$, and $\lambda 5739.7$ lines are fairly unblended for modelling detailed shapes and EWs. The Si III $\lambda 4574.76$ line is weak (the weakest line of the $3 s 4 p$ triplet lines) in MWC 314 , and we do not attempt to fit this line. The $\lambda 4552.62$ and $\lambda 4567.82$ triplet $\left(3 \mathrm{~S}-3 \mathrm{P}^{\circ}\right)$ lines belong to the $3 \mathrm{~s} 4 \mathrm{~s}-3 \mathrm{~s} 4 \mathrm{p}$ Si III atomic configuration with both sharing the lower energy level of $\chi_{\text {low }}=$ $19.01 \mathrm{eV}$ (with $\chi_{\text {up }}=21.7 \mathrm{eV}$ ). The $\lambda 5739.7$ line is a singlet $\left(1 \mathrm{~S}-1 \mathrm{P}^{\circ}\right)$ transition with slightly larger $\chi_{\text {low }}=19.72 \mathrm{eV}$ (and $\chi_{\text {up }}=21.88 \mathrm{eV}$ ). The latter line is also weakest of the three 


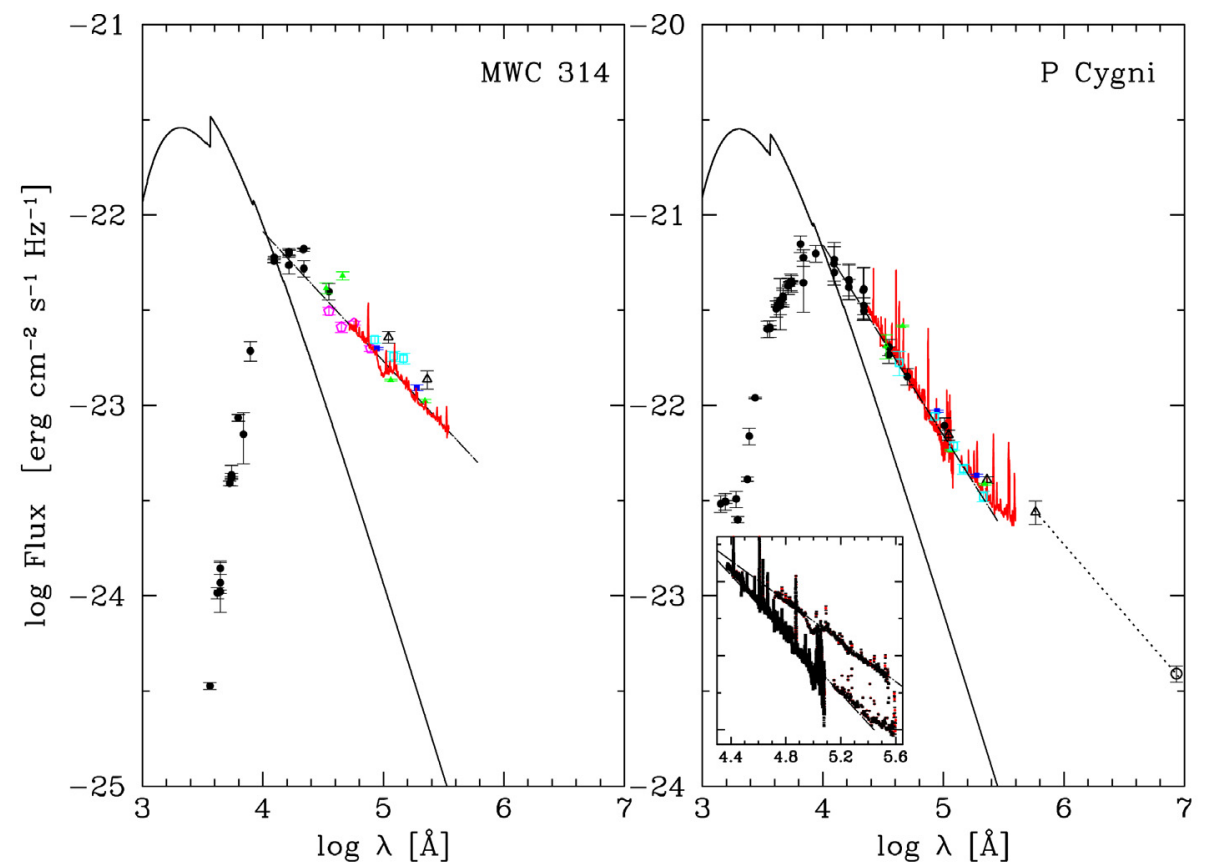

Fig. 11. Comparison of the SED of MWC 314 and LBV P Cygni reveals very similar slopes (dash-dotted and dotted lines) of the near-IR and IR flux density in these early B-type Ia supergiants. Broadband photometric observations (solid black dots), including from five space missions (open and solid triangles, open and solid boxes, and open pentagonal symbols), are compared to Spitzer and ISO spectra (solid drawn line with spikes, red in online version). The sub-panel in the right-hand panel compares the slopes of the Spitzer spectrum of MWC 314 (upper curve) with the ISO and Spitzer spectra of P Cyg (see text). The observed IR flux excesses compared to the photospheric continuum fluxes (smoothly drawn solid lines) are caused by free-free emission in an expanding stellar wind. lines, and we adopt $\log (g f)=-0.096$ from the NIST database. We also adopt $\log (g f)=0.292$ for $\lambda 4552.62$, and $\log (g f)=$ 0.068 for 24567.82 , because the NIST values are provided with the $B^{+}$accuracy flag. We developed a Si III atom model with 12 energy levels, which range from $0 \mathrm{eV}$ to $21.88 \mathrm{eV}$, and a (Si IV) continuum level. The upper levels of the triplet lines are treated as separate levels, while the three levels of $3 \mathrm{~s} 3 \mathrm{p}$, $3 \mathrm{p}^{2}$, and $3 \mathrm{~s} 3 \mathrm{~d}$ are combined into superlevels. We solve the detailed balance for 29 selected lines (and 12 continua), of which some can directly (de-)excite the upper and lower energy levels of the observed Si III triplet and singlet lines. The (non-LTE) lambda iterations to detailed balance are performed, with the temperature of the stellar continuum radiation field $\left(T_{\text {rad }}\right)$ set equal to $18 \mathrm{kK}$, until variations in the line source functions decrease to below $\sim 1 \%$. For example, our Si III atom model also includes the $3 \mathrm{p}^{2}{ }^{1} \mathrm{~S}$ and $2 \mathrm{~s} 3 \mathrm{~d}^{1} \mathrm{D}$ energy levels between the upper and lower levels of the triplet lines. The collisional and radiative equilibrium calculations therefore also include the $\lambda 4338.5$ line, which has the upper level in common with the singlet line. We observe a weak $\lambda 4338.5$ line in the spectrum of MWC 314, but it is too noisy for detailed fits. The $\lambda 9323.89$ and $\lambda 10525.5$ lines are also included (connecting ${ }^{1} D-{ }^{1} P^{\circ}$ and ${ }^{1} \mathrm{D}-{ }^{3} \mathrm{P}^{\circ}$ levels), but these lines are outside our observed wavelength range. We also compute the $\lambda 4325.888\left({ }^{3} \mathrm{~S}-{ }^{1} \mathrm{P}^{\circ}\right)$ and $\lambda 6173.61\left({ }^{1} \mathrm{~S}-{ }^{3} \mathrm{P}^{\circ}\right)$ lines but do not detect them in the observed spectra; the $\log (g f)$ values are below -2.6 .

We employ a grid of plane-parallel atmosphere Kurucz models computed with the opacity distribution functions of Castelli $\&$ Kurucz (2013). The Kurucz atmosphere models are utilised in MULTI for performing the detailed Si III line profile calculations. The MULTI code was developed for non-LTE modelling of detailed line profiles using input multi-level atom models that can be modified for energy levels, continua, selected line transitions, branching ratios, cross sections, etc. The transfer code is very useful for testing the effects of various atomic input parameters on the theoretical line profiles. We find it particularly advantageous for constructing atom models of the higher ions Si II and Si III, because the input atomic data are collected and combined from various databases.
We adopt mean solar metallicity $[\mathrm{M} / \mathrm{H}]=0.0$ Kurucz atmosphere models and use solar abundance values for silicon. The model $T_{\text {eff }}$ ranges from $17 \mathrm{kK}$ to $21 \mathrm{kK}$ in steps of $1000 \mathrm{~K}$. The model surface gravity values, $\log g$, range from 2.5 to 4.0 in steps of 0.5 dex. We compute the Si III lines for two projected microturbulence velocity $\left(\zeta_{\mu}\right)$ values of 5 and $10 \mathrm{~km} \mathrm{~s}^{-1}$. Next, the computed lines are broadened with an instrumental profile of $R=80000$, and the projected rotational velocity $V_{\text {rot }} \sin i$ range from 40 to $100 \mathrm{~km} \mathrm{~s}^{-1}$ in steps of $5 \mathrm{~km} \mathrm{~s}^{-1}$. A least squares fit to the observed profiles of the three Si III lines reveals that $V_{\text {rot }} \sin i$ does not exceed $50 \mathrm{~km} \mathrm{~s}^{-1}$. We obtain best fits for atmosphere models of $\left(T_{\text {eff }}, \log g\right)=(17 \mathrm{kK}, 2.2),(18 \mathrm{kK}$, $2.6)$, and $(19 \mathrm{kK}, 3.0)$ in the case that $\zeta_{\mu}=5 \mathrm{~km} \mathrm{~s}^{-1}$. Figure 10 shows the observed Si III line profiles overplotted with the computed profiles for $17 \mathrm{kK}, 18 \mathrm{kK}$, and $19 \mathrm{kK}$. The boldly drawn lines show profiles computed for $\log g=2.5$, while the thin drawn lines for $\log g=3.0$. Models with $T_{\text {eff }}$ above $19 \mathrm{kK}$ yield EW values incompatible with the observed values. This is also the case for higher $\zeta_{\mu}=10 \mathrm{~km} \mathrm{~s}^{-1}$, requiring $T_{\text {eff }}$ values well above $20 \mathrm{kK}$. Models with $T_{\mathrm{eff}} \geq 20 \mathrm{kK}$ are not supported, because we do not observe the optical Si IV lines. Note that the wavelength regions of the Si IV lines are void of strong emission lines, so that these absorption lines ought to be observed in the case that $T_{\text {eff }}$ of MWC 314 would exceed $20 \mathrm{kK}$. Our modelling of the Si III absorption spectrum of MWC 314 indicates a minimum $\log g \simeq 2.2$ dex. Smaller $\log g$-values would require $T_{\text {eff }}$ below $17 \mathrm{kK}$, which is not compatible with several high-excitation $\mathrm{Ne}$ I absorption lines that we also observe in the spectrum.

\subsection{Spectral energy distribution}

We compare the SEDs of MWC 314 and the (first discovered) LBV P Cygni. The comparison is justified by very similar optical spectra (Polidan 1992). de Jager (2001) provides an overview

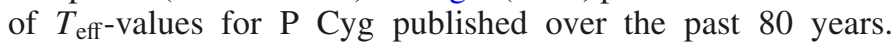
For 2000 , he adopts $T_{\text {eff }}=18600 \mathrm{~K}$ with an estimated uncertainty of a few $100 \mathrm{~K}$. The effective acceleration $\left(g_{\mathrm{eff}}\right)$ in the atmosphere and wind is dominated by radiative forces (more than 10 times the gravity acceleration), and values for $\log g_{\text {eff }}$ 
Table 3. Broadband space-based IR flux density observations of MWC 314 and P Cygni from five missions.

\begin{tabular}{|c|c|c|c|c|c|c|c|}
\hline \multirow[b]{2}{*}{ Mission } & \multirow[b]{2}{*}{ Band } & \multirow[b]{2}{*}{$\begin{array}{c}\lambda_{\mathrm{eff}} \\
{[\mu \mathrm{m}]}\end{array}$} & \multicolumn{2}{|c|}{ MWC 314} & \multicolumn{2}{|c|}{ P Cygni } & \multirow[b]{2}{*}{ Ref } \\
\hline & & & $F_{v}$ & $\begin{array}{r}\sigma\left(F_{v}\right) \\
\times 10^{-23} \text { [er }\end{array}$ & $\begin{array}{c}F_{v} \\
\mathrm{~s}^{-1} \mathrm{~cm}^{-2}\end{array}$ & $\begin{array}{l}\sigma\left(F_{v}\right) \\
\left.\mathrm{Hz}^{-1}\right]\end{array}$ & \\
\hline \multirow[t]{3}{*}{ IRAS } & F12 & 11.0357 & 2.2800 & 0.1596 & 6.9800 & 0.4188 & 1 \\
\hline & F25 & 23.0724 & 1.3700 & 0.1507 & 0.1624 & 4.0600 & 1 \\
\hline & F60 & 58.1904 & - & - & 0.3850 & 2.7500 & 1 \\
\hline \multirow[t]{2}{*}{ AKARI } & S9W & 8.85203 & 1.9930 & 0.0276 & 9.3020 & 0.0930 & 2 \\
\hline & L18W & 18.9186 & 1.2380 & 0.0432 & 0.0673 & 4.2900 & 2 \\
\hline \multirow[t]{5}{*}{ MSX } & B1 & 4.29352 & - & - & 16.7399 & 2.4105 & 3 \\
\hline & A & 8.48032 & 2.2140 & 0.0907 & 8.7429 & 0.3584 & 3 \\
\hline & $\mathrm{C}$ & 12.1516 & 1.8070 & 0.1084 & 6.1250 & 0.3123 & 3 \\
\hline & D & 14.6783 & 1.7620 & 0.1145 & 4.6300 & 0.2824 & 3 \\
\hline & E & 21.5008 & - & - & 3.3230 & 0.2126 & 3 \\
\hline \multirow{4}{*}{ IRAC } & 36 & 3.54391 & 1.3075 & 0.1459 & - & - & 4 \\
\hline & 45 & 4.48406 & 2.5730 & 0.1630 & - & - & 4 \\
\hline & 58 & 5.71638 & 2.6970 & 0.0678 & - & - & 4 \\
\hline & 80 & 7.82825 & 1.9870 & 0.0668 & - & - & 4 \\
\hline \multirow[t]{4}{*}{ WISE } & W1 & 3.34597 & 4.1277 & 0.2813 & 20.5167 & 2.9667 & 5 \\
\hline & W2 & 4.59522 & 4.7897 & 0.2470 & 26.0801 & 0.2608 & 5 \\
\hline & W3 & 11.5485 & 1.3569 & 0.0150 & 5.7990 & 0.0580 & 5 \\
\hline & W4 & 22.0789 & 1.0545 & 0.0223 & 3.8570 & 0.0386 & 5 \\
\hline
\end{tabular}

References. (1) Helou \& Walker (1988); (2) Ishihara et al. (2010); (3) Egan et al. (2003); (4) IPAC (2008); (5) Cutri \& et al. (2012).

ranging from 1.2 to 2.05 are derived from spectral lines that form in different parts of the wind of P Cyg. These values are comparable to $\log g=2.2-2.6$ and $T_{\text {eff }}=17-18 \mathrm{kK}$ that we compute for MWC 314 in Sect. 4.2 using non-LTE fits of three photospheric absorption lines.

Figure 11 shows the flux density of broadband (BB) photometric observations of MWC 314 (left-hand panel) and P Cyg (right-hand panel) that we collect from the literature. Solid black dots mark published flux values (we convert to flux density) observed in the Johnson (Kharchenko 2001; Morel \& Magnenat 1978; Richmond 2007; Mermilliod 2006; Ofek 2008; Ducati 2002), Strömgren (Hauck \& Mermilliod 1998), and Vilnius (Straizys et al. 1989) photometric systems and in various 2MASS (Cutri et al. 2003), TD 1 (Humphries et al. 1976), OAO 2 (Code et al. 1980), Tycho 2 (Høg et al. 2000), HIPPARCos (Perryman \& ESA 1997), and SDSS (Adelman-McCarthy et al. 2008) photometric bands. The BB flux density values are plotted at the effective wavelength $\lambda_{\text {eff }}$ of the passbands. We observe the SED maximum around $\sim 2 \mu \mathrm{m}$ in MWC 314 , longward of the SED maximum of P Cyg. The latter maximum occurs shortward of $1 \mu \mathrm{m}$. This was also observed by Miroshnichenko (1996) with $U B V R I J H K$ photometry (see his Fig. 1). He computes $A_{\mathrm{V}}=5 \mathrm{~m} .5$ from model optical SEDs with $T_{\star}=20 \mathrm{kK}$ and $\log g=2.5$ for MWC 314 . The $E_{B-V}=1.83$ signals a very reddened luminous B-type star with intrinsic colours close to P Cyg.

For a more detailed study of the SEDs longward of $3 \mu \mathrm{m}$ we complement Fig. 11 with photometric data from the AKARI (solid boxed symbols), MSX (open boxed symbols), WISE (solid triangles), and Spitzer-IRAC missions (open pentagonal symbols longward of $3 \mu \mathrm{m}$ in MWC 314). The (linear) absolute IR flux densities and errors are listed in Table 3.

We investigate the SED slopes of both stars with NASA Spitzer Space Telescope and ESA Infrared Space Observatory (ISO) spectra. The Spitzer-IRSX spectrum of MWC 314 is shown in Fig. 11. It was observed between $5.21 \mu \mathrm{m}$ and $34.98 \mu \mathrm{m}$ on 6 Nov. 2008 ${ }^{1}$. Ardila et al. (2010) note the presence of the broad silicate dust absorption feature at $10 \mu \mathrm{m}$ that can originate in the interstellar medium. The feature is weak

\footnotetext{
1 sha.ipac.caltech.edu/applications/Spitzer/SHA
}

in MWC 314 compared to other LBVs and LBV candidates. LBV candidate Cyg OB2 No. 12 (B5 Ie) has a very strong silicate absorption feature (see their Fig. 5A), indicating its large dust extinction may be of circumstellar nature. The right-hand panel of Fig. 11 shows the ISO flux density spectrum of P Cyg (solid drawn line with spikes) between $2.36 \mu \mathrm{m}$ and $12 \mu \mathrm{m}$. It was observed with ISO-SWS on 17 Oct. $1996^{2}$. The ISO spectrum longward of $12 \mu \mathrm{m}$ is not shown, because the absolute flux errors rapidly exceed $1 \mathrm{Jy}$. We overplot the Spitzer-IRSX spectrum of P Cyg that is observed between $14.0 \mu \mathrm{m}$ and $39.72 \mu \mathrm{m}$ (on 17 Dec. 2008 for SIRTF IRS Calibration Program), which have small flux errors below $0.05 \mathrm{Jy}$ shortward of $37 \mu \mathrm{m}$. The Spitzer and ISO spectra show that the IR SED slope of P Cyg is indeed only slightly steeper than the Spitzer spectrum of MWC 314 that we also observe with AKARI, MSX, and WISE photometry.

The sub-panel in the right-hand panel of Fig. 11 compares the Spitzer spectrum of MWC 314 and the ISO and Spitzer spectrum of P Cyg. The spectrum flux values are plotted with the flux errors, provided by the ISO and Spitzer calibration pipelines. The P Cyg spectrum is shifted down by 0.9 for detailed comparison to the spectrum slope in MWC 314. The IR spectrum of MWC 314 can be well modelled by $\lambda^{-0.68}$ up to $22 \mu \mathrm{m}$ (dashdotted line). On the other hand, the IR spectrum of P Cyg is best fit with a steeper slope. We find that changes in the (inverse lambda) power of $\sim \pm 0.05$ significantly alter the model slope. The best fit to the continuum flux level that we observe in both spectra yields $\lambda^{-0.68 \pm 0.05}$ in MWC 314 and $\lambda^{-1.00 \pm 0.10}$ for P Cyg.

The IRAS $12 \mu \mathrm{m}, 25 \mu \mathrm{m}$, and $60 \mu \mathrm{m}$ fluxes in Fig. 11 are high-quality observations and reveal IR SED slopes that are almost identical to the Spitzer or/and ISO spectra. In P Cyg, however, the $60 \mu \mathrm{m}$ BB flux exceeds the $\lambda^{-1.0}$ flux density dependence, and the Spitzer spectrum reveals a decrease of the gradient in flux density towards longer wavelengths. The IR SED slope, connecting the IRAS $60 \mu \mathrm{m}$ and the JCMT-Scuba submillimetre (open circle shown at $859 \mu \mathrm{m}$ ) flux values (Di Francesco et al. 2008) in P Cyg, yield a far-IR slope of $\lambda^{-0.73}$ (dotted drawn line).

2 iso.esac.esa.int/ida 



Fig. 12. Three strong He I lines reveal remarkable variability of shape with orbital phase. They show single-peaked emission around the systemic velocity. The blue-shifted and variable absorption is strongest at $\phi=0.65-0.85$. The lines are variable P Cyg-type line profiles. The peculiar P Cyg line shape is always observed in He I $\lambda 5876$.

In Fig. 11, we plot the photospheric continuum flux distribution of both stars. We compute photospheric fluxes using Kurucz models (see Sect. 4.2) of $T_{\text {eff }}=18 \mathrm{kK}$ and $\log g=2.5$ for MWC 314 and $T_{\text {eff }}=19 \mathrm{kK}$ and $\log g=2.5$ for P Cyg. The photospheric spectra are almost identical longward of the SED maxima, following a Rayleigh-Jeans tail $\left(\lambda^{-2}\right)$. Between $2 \mu \mathrm{m}$ and $100 \mu \mathrm{m}$, the SEDs however substantially exceed the photospheric model flux values. The excess flux is attributed to free-free (continuum) radiation emerging from the ionised and expanding winds of massive hot stars with emission lines (provided scattering by dust grains is negligible). Lamers \& Cassinelli (1999) show that the predicted energy distribution is a power law of $\lambda^{-0.61}$ for radio free-free emission from an isothermal wind with constant velocity (see their Eq. (2.20)). The shape of the SED shortward of $100 \mu \mathrm{m}$ depends on the gas kinetic temperature and density in the layers where the wind velocity increases outwards. In P Cyg, the radiative wind driving mechanism yields a "beta"-power law with $\beta \simeq 1$ (i.e., Najarro et al. 1997; Najarro 2001). Since we observe comparable near-

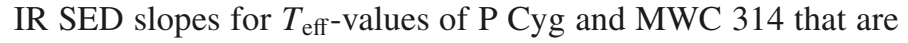
nearly identical and compute integrated total excess fluxes that largely exceed the photospheric flux by similar factors, we can adopt the $\beta=1$ wind velocity structure for MWC 314 . (We further discuss the values of $\beta$ for 3-D RT modelling in Sect. 5.3.)
The conservation of wind momentum requires $\rho(r)=\rho_{0} / r^{2}$ for the smooth wind density structure of MWC 314. However, the spherical wind geometry is not supported since we find that MWC 314 is a short-period binary system, and the values of $\dot{M}$ and $v_{\infty}$ observed in P Cyg cannot readily be assumed for MWC 314. In Sect. 5, we measure $v_{\infty}$ from detailed radiative transfer fits to He I P Cyg-type line profiles in MWC 314. The $3 \mathrm{D}$ model of the radial wind velocity structure employs a modified $\beta$-law that accounts for the orbital motion of the primary star observed at different orbital phases in the rest frame of the observer.

\subsection{Emission line variability}

\subsubsection{He I P Cyg lines}

The strong He I $\lambda 4471, \lambda 5876, \lambda 6678$ emission lines show remarkable variability in the spectrum of MWC 314 . The normalised line fluxes and dynamic spectra are shown with $\phi$ in the heliocentric radial velocity scale in Fig. 12. The He I lines are always single-peaked with emission flux maxima around the systemic $\gamma$-velocity. The high-resolution spectra reveal variable blue-shifted absorption which becomes strongest during $\phi=0.65-0.85$. The He I lines show variable P Cyg-type line profiles. The P Cyg profile is always observed in He I $\lambda 5876$. 

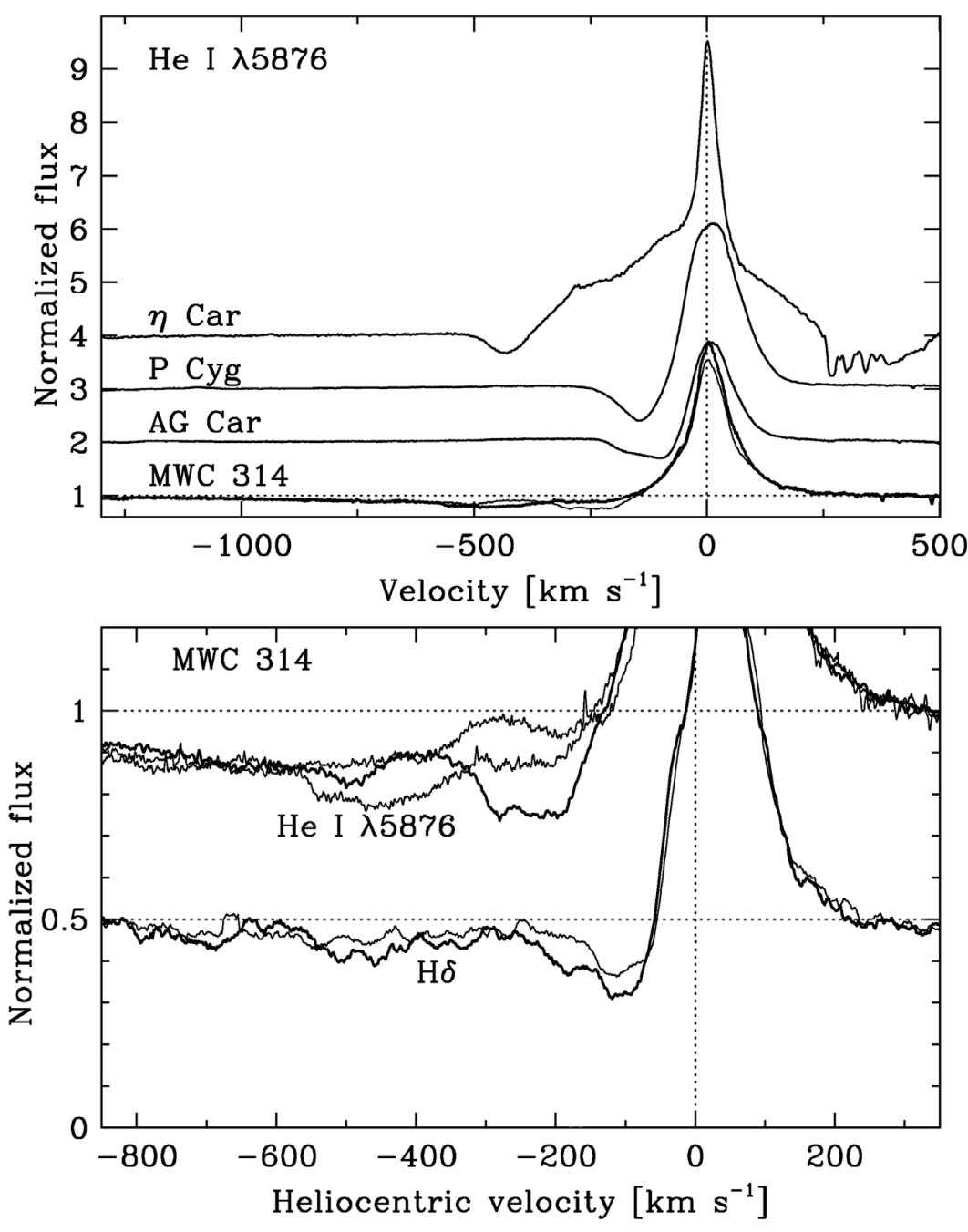

Fig. 13. Top panel: He I $\lambda 5876$ lines in three LBVs, $\eta$ Car, $\mathrm{P}$ Cyg, and AG Car are compared to MWC 314. We observe blue-shifted wind absorption beyond $1000 \mathrm{~km} \mathrm{~s}^{-1}$ in MWC 314. Bottom panel: blue-shifted absorption reveals strong variability below $600 \mathrm{~km} \mathrm{~s}^{-1}$ (solid thick and thin lines), which we also observe in $\mathrm{H} \delta$.
In Fig. 13, we compare the He I $\lambda 5876$ profiles of three LBVs, $\eta$ Car, P Cyg, and AG Car, to the line in MWC 314. The top panel plots two spectra of MWC 314 (solid thick and thin drawn lines) to $1300 \mathrm{~km} \mathrm{~s}^{-1}$. We observe the blue-shifted wing absorption well beyond $1000 \mathrm{~km} \mathrm{~s}^{-1}$ in MWC 314, while it is observed to $\sim 500 \mathrm{~km} \mathrm{~s}^{-1}$ in the other LBVs. Groh et al. (2007) find a similar behaviour in the He I $\lambda 10830$ line of MWC 314 with the absorption wing extending to $1800 \mathrm{~km} \mathrm{~s}^{-1}$ at certain epochs. Richardson et al. (2010) discuss P Cyg type absorption near $-500 \mathrm{~km} \mathrm{~s}^{-1}$ in $\mathrm{H} \alpha$ of $\eta$ Car which could be possibly due to binary interaction around periastron passage. The bottom panel of Fig. 13 shows strong absorption variability in He I $\lambda 5876$ of MWC 314 for wind velocities below $500 \mathrm{~km} \mathrm{~s}^{-1}$ (solid thick and thin drawn lines). We observe similar blue-shifted absorption variability in the higher $\mathrm{H}$ Balmer emission lines, such as $\mathrm{H} \delta$ (solid thick and thin drawn lines).

The blue-shifted absorption observed in the He I lines of MWC 314 is produced in a fast-expanding warm wind surrounding the primary, a massive hot supergiant. The expanding wind opacity becomes best visible in high-temperature (i.e., $\mathrm{He}$ I) lines because the absorption line formation region is located relatively close to the primary within a few $R_{\star}$ above the stellar photosphere (see Sect. 5). The He I line emission emerges from a wind region close to the supergiant and does not considerably fill in the blue-shifted wind absorption. The strong emission portions of the lower $\mathrm{H}$ Balmer series lines (that form at lower excitation temperatures), on the other hand, emerge from a much more extended circumbinary wind envelope that completely squelches blue-shifted absorption. The variable blue-shifted P Cyg absorption in the He I lines results from the increase in line-of-sight wind opacity in front of the hot star during orbital phases of fastest approach to the observer. During $\phi=0.65-0.85$, the radial velocity of the blue-shifted wind absorption is most separated from the velocity of the emission line, and hence the overall P Cyg line shape becomes also best visible. The orbitally modulated absorption that we observe in the He I lines signals an asymmetrically extended wind envelope with enhanced density, which is oriented towards the wind region that leads the orbit of the primary. Interestingly, similar behaviour is discussed by (Tubbesing et al. 2002) for RMC 81 with higher $v_{\infty}$ at orbital phases when the secondary is in front of the primary, which is observed in the higher Balmer lines and metallic P Cyg profiles. They attribute this to an increase in the mass-loss rate in the direction of the secondary. This is caused by tidal interaction with the stellar wind, which can result in a focused wind (Friend \& Castor 1982). In Sect. 5, we develop a 3-D model of the binary wind structure in MWC 314, which fits the observed P Cyg He I line variability.

\subsubsection{H Balmer and Paschen emission lines}

We also investigate the hydrogen Balmer and Paschen emission lines in relation to the spectroscopic orbital variability of MWC 314. The normalised $\mathrm{H} \alpha$ and $\mathrm{H} \beta$ line fluxes are plotted 
according to the orbital phases in the top panels of Fig. 14. Both $\mathrm{H}$ lines are the strongest emission lines in the optical and near-IR spectrum. The FWHM of the $\mathrm{H} \alpha$ line is $\simeq 200 \mathrm{~km} \mathrm{~s}^{-1}$, while $\simeq 150 \mathrm{~km} \mathrm{~s}^{-1}$ in $\mathrm{H} \beta$. The FWHM values gradually decrease towards the higher Balmer series lines, while the unblended $\mathrm{H} \mathrm{Pa} 14$ emission line has a $F W H M \simeq 100 \mathrm{~km} \mathrm{~s}^{-1}$ (bottom panels). The line wings of $\mathrm{H} \alpha$ extend well beyond $\pm 300 \mathrm{~km} \mathrm{~s}^{-1}$. The high-resolution profiles reveal that the violet emission line wing is always more extended than the red one. Towards the higher $\mathrm{H}$ Balmer lines, stronger absorption extending to $\sim 300 \mathrm{~km} \mathrm{~s}^{-1}$ becomes more apparent in the violet line wing. The $\mathrm{H} \delta$ and Pa14 lines in the bottom panels of Fig. 14 exhibit enhanced blueshifted absorption during $\phi=0.65-0.85$, similar to the He I lines in Fig. 12. The P Cyg-type line shape of the higher Balmer and Paschen series lines is also noticeable, although it is less pronounced than in the He I lines.

We find clear evidence that the continuum normalised emission line maxima of $\mathrm{H} \alpha$ and $\mathrm{H} \beta$ are variable and orbitally modulated. Figure 15 shows the upper portion of the continuum normalised $\mathrm{H} \alpha$ (bottom panel) and $\mathrm{H} \beta$ (top panel) emission lines. The line flux maxima are shifted to the orbital phase $\phi$ and marked with the spectrum number. The normalised $\mathrm{H} \beta$ line flux maxima vary between 6.4 and 7.3, while $\mathrm{H} \alpha$ varies between 22.8 and 25.6 (Table 1). Both lines show largest flux maxima for $\phi \simeq 0.65$ (spectrum Nos. 6 and 16), and smallest flux maxima for $\phi \simeq 0.32$ (Nos. 13, 15, and 7). The $\phi$-dependent $\mathrm{H} \alpha$ (open symbols) and $\mathrm{H} \beta$ (filled symbols) flux maxima are shown scaled in the top panel of Fig. 16. For comparison purposes, the normalised $\mathrm{H} \alpha$ line flux maxima are divided by 24 , and the $\mathrm{H} \beta$ maxima by 6.75 . We measure emission line EW values of $120-150 \AA$ for $\mathrm{H} \alpha$ and $35-45 \AA$ for $\mathrm{H} \beta$. The $\mathrm{H} \alpha$ line is however mutilated by strong telluric absorption lines at some orbital phases (see the bottom panel of Fig. 15), which can slightly perturb the EW-orbital phase dependence.

We also find a clear correlation between the continuum normalised emission flux maxima observed in the $\mathrm{H} \alpha$ \& $\mathrm{H} \beta$ lines and the $V$-brightness curve of MWC 314 . The $V$-band brightness maximum around $\phi=0.3$ in the bottom panel of Fig. 5 yields $\mathrm{H} \alpha$ and $\mathrm{H} \beta$ emission line maxima that become apparently weaker after continuum flux normalisation compared to orbital phases when the $V$-continuum brightness is dimmest $(\phi=0.65)$. The orbital variability of about $\pm 7 \%$ in the continuum normalised emission flux maxima of both lines (top panel of Fig. 16) is fully accounted by the photometric $V$-continuum flux changes of about \pm 0.05 that we observe in the ASAS light curve of MWC 314. The product of the visual flux (we compute from $V$ with orbital phase) and the continuum normalised emission line maxima is almost invariable. The near constancy of the absolute $\mathrm{H} \alpha$ and $\mathrm{H} \beta$ emission line fluxes therefore indicates that the $\mathrm{H}$ emission line formation regions are not substantially influenced by orbital motion. It signals a very extended formation region for the $\mathrm{H} \alpha$ and $\mathrm{H} \beta$ emission lines. We think therefore that both $\mathrm{H}$ emission lines form in a very extended circumbinary envelope around MWC 314 in which the bulk of the line emission regions are not substantially modulated by the orbital motion.

\subsubsection{Orbital modulation of permitted and forbidden emission lines}

The optical spectrum of MWC 314 shows many prominent metal emission lines of Fe II, Ti II, and Si II. Figure 17 shows a portion of the spectrum with the permitted Fe II $\lambda 6416.9, \lambda 6432.7$, and $\lambda 6456.4$ emission lines. The normalised flux spectra are shifted upwards according to the orbital phase ( $\phi$ and spectrum
No. are marked). The Doppler shift of the Ne I $\lambda 6402.2$ absorption line with $\phi$ is shown by the vertically plotted curved line (dotted). The three Fe II lines mostly show double and occasionally triple flux maxima. It is very remarkable to observe that the detailed shapes of the emission lines almost perfectly depend on $\phi$. The flux maxima of the blue and red emission peaks vary with $\phi$; the red maximum exceeds the blue peak for $\phi=0.95$ to $\sim 0.5$, while the blue peak exceeds the red one for $\phi=0.5$ to 0.95 . The bottom panel of Fig. 16 shows the ratio of blue/red (B/R) emission flux maxima with $\phi$ for Fe II $\lambda 6433$ (filled triangles) and Fe II $\lambda 6456$ (open boxed symbols). The B/R flux ratios are provided in the last two columns of Table 1. Notice the strong correlation to the (normalised) intensity variations in the $\mathrm{H} \alpha$ and $\mathrm{H} \beta$ emission line maxima in the top panel of Fig. 16. The $\mathrm{B} / \mathrm{R}$ flux ratio variations of about $\pm 10 \%$ that we observe, however, result from intrinsic changes in the detailed profile shape of the Fe II emission lines with $\phi$.

We also observe orbital modulation over short $\phi$-intervals. For example, the Fe II lines from $\phi=0.405$ to 0.462 reveal three separate flux maxima in the central emission cores (see spectrum Nos. 12, 1, 8, and 4). We compare the spectra of 5 June 2009 (No. $1 ; \phi=0.440$ ) and 4 Oct. 2010 (No. 8; $\phi=0.444$ ) and observe nearly identical emission line shapes. Both spectra are however observed $16 \mathrm{~m}$ apart or separated in time by $\sim 8$ orbital periods. It is of note that very weak and stable spectral features close to the stellar continuum almost exactly repeat over time (i.e., observed around $6445 \AA$ in Fig. 17), signaling that orbital modulation strongly determines the spectral line variability that we observe in MWC 314. The triple-peaked metal emission lines of MWC 314 are very remarkable spectral features. They are clearly linked to the spectral variability with orbital phases and will be investigated in the future. The similarity to the phenomenon in the spectra of Be-stars is also striking and may signal a common emission line formation mechanism, which is due to the circumbinary disc of a close binary system. For example, Schaefer et al. (2010) mention the triple emission line maxima can be caused by a one-armed oscillation or precessing disc in Be-star $\zeta$ Tau.

We investigated the detailed shapes and FWHM widths of various permitted and forbidden emission lines in the highresolution HERMES spectra of MWC 314. Very striking are the double-horned shape of many permitted and forbidden emission lines. For example, the wings of Fe II $\lambda 6433$ in the right-hand bottom panel of Fig. 18 are very steep, while the line core is almost flat around the central flux minimum. Similar detailed line shapes are observed in [Fe II] $\lambda 7155$ and [Fe II] $\lambda 8616$ in the top and bottom right-hand panels of Fig. 18. We do not measure any significant Doppler shift in the position of the steep emission line wings with $\phi$. The line wings are static within $\pm 3 \mathrm{~km} \mathrm{~s}^{-1}$.

Three [N II] emission lines $\lambda 6583, \lambda 5754$, and $\lambda 6548$ are observed. The first two are shown with $\phi$ in the top and bottom left-hand panels of Fig. 19. The [N II] lines are single peaked with almost flat-topped emission maxima that can occasionally become double peaked (i.e., [N II] 25754). The [N II] emission lines are formed in nebulae around many massive stars, signaling CNO processed material. The lines are observed in a number of LBVs with $T_{\text {eff }}$ that are very similar to MWC 314 like P Cyg, AG Car, HR Car, and HD 168625. Cooler hypergiants such as HR 8752 (currently of A-type), also reveal the [N II] lines (Lobel et al. 2013). We searched for other forbidden emission lines in the spectrum but failed to detect the [O I] $\lambda 5577$ and $\lambda 6300$ lines reported by Kurchakov \& Rspaev (2005). There is no indication of stellar emission at these line rest wavelengths. The permitted O I triplet $\lambda 7771-5$ lines, on the other hand, reveal 


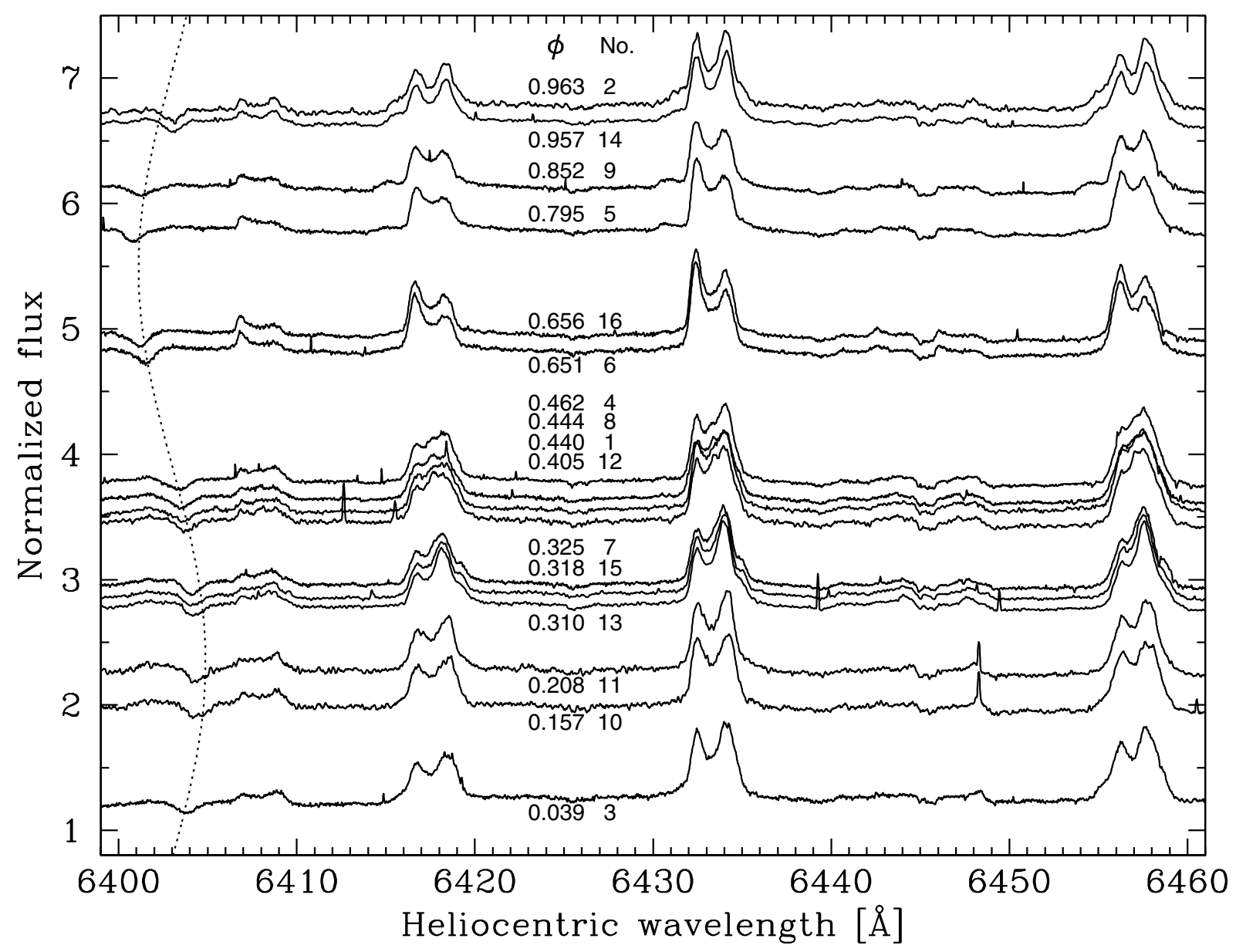

Fig. 17. Portion of the MWC 314 spectrum showing permitted Fe II $\lambda 6416.9, \lambda 6432.7$, and $\lambda 6456.4$ emission lines. The spectra are shifted upwards according the orbital phase (the labels show $\phi$ and spectrum Nos.). The orbital Doppler shift of the Ne I $\lambda 6402.2$ absorption line is shown by the vertical curved line (drawn dotted). We show in this paper that the detailed shapes of the emission lines precisely depend on $\phi$, or are mainly caused by orbital modulation. For example, No. $1(\phi=0.440)$ and No. $8(\phi=0.444)$ reveal almost identical spectra, we observe however $16 \mathrm{~m}$ apart, or separated by $\sim 8 \times P$.

a strong and broad P Cyg shape, while O I 18446 is a prominent double-peaked emission line. We also observe [Ca II] $\lambda 7291.506$ and $\lambda 7323$ emission lines, although they are heavily mutilated by overlying narrow telluric $\mathrm{O}_{2}$ doublet lines. The $\mathrm{Ca}$ II $\mathrm{H} \& \mathrm{~K}$ lines also show P Cyg profiles, while the near-IR Ca II triplet lines are prominently double- or multiple-peaked emission lines. The S II $\lambda 6347$ line shows a shallow and extended P Cyg line shape. We also search for forbidden metal emission lines of [Ti II] and [Cr II] but fail to identify them. We employ the [Ti II] line list in Table 2 of Hartman et al. (2005) (we convert to air wavelengths) but do not detect any [Ti II] emission lines in MWC 314.

Table 4 provides a list of emission lines that we investigate in the HERMES spectrum of MWC 314 of Sept. 52009 (spectrum No. 2 in Table 1), when the radial velocity is close to the $\gamma$-velocity. It also lists the heliocentric radial velocities of the blue and red emission maxima of double-peaked lines.

\subsubsection{Emission line formation}

Muratorio et al. (2008) find that the double-peaked metal emission lines in MWC 314 are consistent with line formation in a rotating disc. They measure the blue and red component velocities of permitted and forbidden Fe II (Cr II and Ti II) double-peaked emission lines and compute the detailed line shape with a model of a projected Keplerian rotating disc. Interestingly, they also compute that the Fe II emission occurs from $2 \pm 1$ times up to $15 \pm 2 R_{\star}$. The [Fe II] lines form in the same disc at distances to the star between $12 \pm 2$ to $15 \pm 2 R_{\star}$.

We observe that the permitted and forbidden Fe II lines have almost identical FWHM of $\simeq 110 \mathrm{~km} \mathrm{~s}^{-1}$ and comparable differences between blue-and red-peak (radial) velocities of $\sim 65 \mathrm{~km} \mathrm{~s}^{-1}$. The strongest and broadest double-peaked lines in the spectrum of MWC 314 are the near-IR H Paschen emission lines. All $\mathrm{Pa}$ lines reveal substructures in the emission flux maxima (i.e., $\mathrm{H} \mathrm{Pa} 14$ in Fig. 14) with $F W H M \sim 100$ to $140 \mathrm{~km} \mathrm{~s}^{-1}$. In contrast, we observe much smaller FWHM values for the [N II] emission lines that do not exceed $50 \mathrm{~km} \mathrm{~s}^{-1}$, which are considerably less than the $\mathrm{Pa}$ and $\mathrm{Fe}$ II lines.

The FWHM values of the emission lines in MWC 314 are a measure of the (projected) rotation velocity in the line formation regions of a Keplerian disc. Richardson et al. (2011) discuss the formation of double-peaked Fe II, Si II, and Ne II lines in a circumbinary disc of the massive WR+O binary HD 326823. They observe $\mathrm{B} / \mathrm{R}$ variability in these double-peaked emission lines, which are very similar to the orbitally modulated $B / R$ variability that we observe in MWC 314. In HD 326823, the Fe II emission lines form in a circumbinary disc consistent with Keplerian motion required to explain the double-peaked profiles. Richardson et al. (2011) provide an expression for the projected Keplerian rotational velocity in the circumbinary disc gas as a function of 
Table 4. Emission lines in the high-resolution HERMES spectrum of MWC 314. FWHM values are provided for single- and double-peaked lines.

\begin{tabular}{|c|c|c|c|c|}
\hline Line & $\begin{array}{c}\lambda_{0} \\
{[\AA]}\end{array}$ & $\begin{array}{c}F W H M \\
{\left[\mathrm{~km} \mathrm{~s}^{-1}\right]}\end{array}$ & $\begin{array}{l}V_{\text {blue-peak }} \\
{\left[\mathrm{km} \mathrm{s}^{-1}\right]}\end{array}$ & $\begin{array}{l}V_{\text {red-peak }} \\
{\left[\mathrm{km} \mathrm{s}^{-1}\right]}\end{array}$ \\
\hline $\mathrm{H} \alpha$ & 6562.797 & 188 & - & - \\
\hline $\mathrm{H} \beta$ & 4861.323 & 135 & - & - \\
\hline $\mathrm{H} \gamma$ & 4340.462 & 132 & - & - \\
\hline $\mathrm{H} \delta$ & 4101.734 & 117 & - & - \\
\hline H Pa14 & 8598.396 & 134 & -7 & +58 \\
\hline He I & 4471.479 & 91 & - & - \\
\hline He I & 5875.790 & 87 & - & - \\
\hline He I & 6678.149 & 82 & - & - \\
\hline O I triplet & $7772-5$ & - & - & - \\
\hline O I & 8446.359 & 111 & -5 & +56 \\
\hline Fe II & 6416.905 & 123 & -6 & +53 \\
\hline Fe II & 6432.654 & 118 & -7 & +62 \\
\hline $\mathrm{Fe}$ II & 6456.376 & 131 & -5 & +54 \\
\hline $\mathrm{Si}$ II & 6347.10 & 149 & -9 & +85 \\
\hline Si II & 6717.04 & 143 & -8 & +81 \\
\hline [N II] & 5754.59 & 49 & +18 & +39 \\
\hline$[\mathrm{N} \mathrm{II}]$ & 6548.05 & 45 & - & - \\
\hline$[\mathrm{N} \mathrm{II}]$ & 6583.45 & 47 & - & - \\
\hline$[\mathrm{Fe}$ II $]$ & 7155.16 & 93 & -8 & +59 \\
\hline [Fe II] & 8616.95 & 96 & -10 & +58 \\
\hline [Ca II] blends $\mathrm{O}_{2}$ & 7291.47 & 46 & - & - \\
\hline [Ca II] blends $\mathrm{O}_{2}$ & 7323.89 & 40 & - & - \\
\hline
\end{tabular}

Notes. Columns 4 and 5 list heliocentric radial velocities of the blue and red emission maxima of double-peaked lines. $\lambda_{0}$ is the laboratory rest wavelength of the line in air.

distance $r$ from the binary. It follows a $r^{-1 / 2}$-dependence with highest emission speed at the inner disc boundary. We think therefore that the Pa lines in MWC 314 form closer to the binary centre of gravity and at higher gas temperatures, than the metallic emission lines, while the [N II] lines form at longer distances in the outer circumbinary disc regions.

The semi-amplitude $K_{1}$-velocity of $\simeq 84.5 \mathrm{~km} \mathrm{~s}^{-1}$ that we measure in the RV-curve of absorption lines (see Sect. 4.1) is larger than the HWHM and/or half of the difference in B/R-peak velocities of double-peaked emission lines: $\sim 65 \mathrm{~km} \mathrm{~s}^{-1}$ in $\mathrm{Pa}$, $\sim 55 \mathrm{~km} \mathrm{~s}^{-1}$ in Fe II, and $\sim 25 \mathrm{~km} \mathrm{~s}^{-1}$ in [N II] lines. It indicates that the radius of the primary's orbit (producing the photospheric absorption lines) is smaller than the mean line formation region distances of the emission flux maxima in a Keplerian rotating disc.

The orbitally modulated $\mathrm{B} / \mathrm{R}$ variability that we observe in MWC 314 can result from two separate line formation regions. The top and bottom left-hand panels of Fig. 18 show the continuum normalised Si II $\lambda 6347$ line variability with $\phi$. The singlepeaked Si II emission line is static around the $\gamma$-velocity. The detailed shape of this $\mathrm{Si}$ II emission line is however periodically altered by Doppler displaced photospheric absorption (following an S-shape) and yields the double-peaked Si II emission line around $\phi=0.9-0.1$.

We also subtract the average flux profile from the Fe II $\lambda 6456$ and $\lambda 6433$ lines shown in Fig. 18. It yields residual flux spectra with a weak (and sometimes P Cyg-type) absorption line that Doppler shifts and causes the B/R peak variability (bottom panel of Fig. 15). The triple-peaked emission profiles that we observe around $\phi=0.4-0.5$ result from rapid blue-shift in the photospheric line becoming noticeable for RV-values around the $\gamma$ velocity between the blue and red emission line maxima. We find that the orbital modulation of the $\mathrm{B} / \mathrm{R}$ variability observed in double-peaked metal emission lines of MWC 314 can result from photospheric absorption Doppler displacing below the (static) emission line formed in the circumbinary disc. A comparable analysis of the orbitally modulated $\mathrm{B} / \mathrm{R}$ variability observed in Fe II emission lines of HD 326823 is presented in Richardson et al. (2011).

\subsection{Spectroscopic signatures of the companion star?}

The high S/Ns and wavelength coverage of the HERMES spectra from $420 \mathrm{~nm}$ to $900 \mathrm{~nm}$ are useful for searching for possible spectral signatures of the companion star. Interestingly, Ardila et al. (2010) find a pattern of emission lines in the Spitzer spectrum $(R \sim 90)$ of MWC 314. They mention strong He I and He II but weak metallic lines, which are similar to early-type O stars, such as the O5f+ star HD 14947. Their Figs. 8 and 14 mark two weak He II lines at $\simeq 22.5 \mu \mathrm{m}$ and $\simeq 27.7 \mu \mathrm{m}$. However, we do not observe the He II $\lambda 4685.7$ line in the HERMES spectra. The spectral region around $4686 \AA$ is almost continuous (flat) without significant emission or absorption features.

The bottom spectrum of Fig. 20 shows the Spitzer spectrum of MWC 314 (thick drawn line) between $5 \mu \mathrm{m}$ and $33 \mu \mathrm{m}$. The top spectrum (thin line) shows the theoretical model spectrum computed with CMFGEN. The vertically drawn lines mark the positions of $\mathrm{H}$ I and $\mathrm{He}$ I lines in the synthetic spectrum calculations. We compute strong $\mathrm{H}$ emission lines (from energy levels $11-9$ and $13-10$ ) at $22.38 \mu \mathrm{m}$ and at $27.8 \mu \mathrm{m}$ (from levels 9-8). These H lines are also observed in Spitzer spectra of B5 supergiants, which do not show the He II $\lambda 4685.7$ emission line at mid-B spectral type. Our synthetic spectrum modelling shows that the Spitzer spectrum of MWC 314 is consistent with $T_{\text {eff }} \simeq 17-19 \mathrm{kK}$. We do not observe optical or mid-IR spectral signatures of an O-type star in MWC 314.

\section{3D binary wind modelling}

We find several indications of a non-spherical geometry of the wind and circumstellar environment of MWC 314. First, MWC 314 contains a hot supergiant primary star in a semidetached massive binary system. The orbital modulation of P Cyg absorption provides important new information about the asymmetry of wind geometry around the primary. It is supported by the deformation of the surface of the primary from a spherical shape due to filling of its Roche lobe (see Sect. 4.1). Second, the prominently double-peaked emission line spectrum indicates a circumbinary disc that can result from wind accretion in MWC 314. Therefore, it is not appropriate to employ onedimensional radiative transfer models to analyse the P Cygnitype lines of MWC 314.

In this section, we develop a quantitative $3 \mathrm{D}$ model of the large-scale wind structure around the primary star in MWC 314. The wind velocity and density structure are computed with detailed 3D radiative transfer (RT) fits to the variable $\mathrm{P}$ Cyg profiles observed in the He I lines of Figs. 12 and 13. Our goal is to obtain insights that help to constrain the physical parameters (e.g., wind density contrast) of the accretion flow in the system.

Figure 21 provides a schematic 3D representation of MWC 314. The primary star is drawn at apastron passage (small blue concentric spheres) in orbit (dashed line) around the centre of gravity (central dot). The circumbinary disc is drawn in the equatorial plane of the binary (marked with dotted concentric lines). The inner accretion disc radius (thick drawn ellipse) is drawn around periastron distance of the primary. We investigate wind accretion in MWC 314 combined with the results of the 3D wind modelling in Sect. 6. 


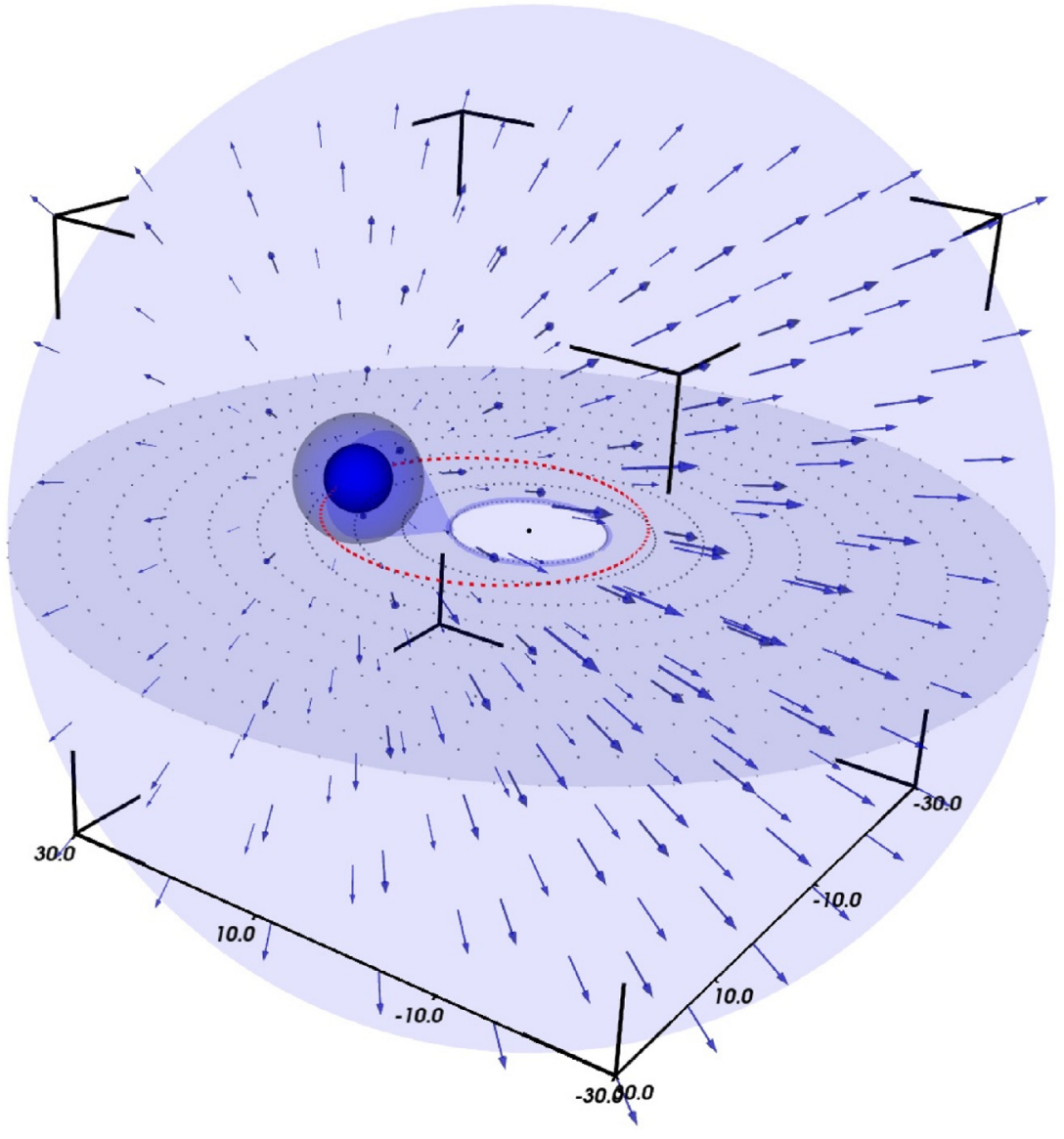

Fig. 21. 3D representation of the wind geometry in MWC 314. The size of the vectors mark the velocity of the asymmetric wind from the primary due to orbital motion. The open righthand arrow marks the observer line of sight. At longer distances the radiatively driven wind becomes symmetric around the centre of gravity (see Sect. 5.1).

\subsection{Wind model velocity structure}

The 3D wind model that we develop does not incorporate a model of an accretion disc in MWC 314. Detailed RT modelling of the variable shape of double-peaked emission lines is outside the scope of this paper. We concentrate here on variable absorption observed in He I P Cyg profiles that form in the supersonically expanding wind of the primary. The stellar wind is driven by the strong radiation field of the hot supergiant to distances much larger than the size of its orbit. The line RT calculations are performed with the WIND3D code (Lobel \& Blomme 2008), using a maximum wind model radius of $30 R_{1}$ around the binary centre of gravity. We adopt a $\beta=1$ radial velocity law $v(r)$ for the wind and a "smooth" wind density structure $\rho(r)=\rho_{0} / r^{2}$ similar to P Cygni (we justify in Sect. 4.3). The density at the base of the wind $\rho_{0}$ is set equal to $\dot{m}_{1} /\left(4 \pi R_{1}^{2} v_{0}\right)$, where $\dot{m}_{1}$ is the primary's wind mass-loss rate of $\sim 10^{-5} M_{\odot} \mathrm{yr}^{-1}$ and $v_{0} \simeq 10 \mathrm{~km} \mathrm{~s}^{-1}$ is the wind velocity around $r=R_{1}$.

First, we perform a Galilean transformation of $v$ from the primary's rest frame to the observer's frame. Our 3D RT calculations utilise a Cartesian model grid. The wind velocity is $v=$ $\left(v_{x}, v_{y}, v_{z}\right)$ in the frame of the primary. The wind velocity component $v_{x}$ is transformed to the observer's frame by $v_{x}^{\prime}=v_{x}+V_{r}$, where the last term is the observed radial velocity depending on $\phi$. The observer's line of sight is in the $(x, z)$-plane of the model grid (i.e., the vertical frontside plane marked in Fig. 21), and we incline the orbital plane by $18^{\circ}$ from the $(x, y)$-plane. The transformation causes Doppler effects due to the primary's orbital motion for the calculation of detailed line profiles in the frame of the observer. The effect of $V_{r}$ on $v_{x}^{\prime}$ is largest in wind regions of $r<2 R_{1}$, where $\left|V_{r}\right| \simeq\left|v_{x}\right|$ (Note that $v_{x}<0$ in the front hemisphere.) At a longer distance from the primary, the wind rapidly accelerates and approaches $v_{\infty}=1200 \mathrm{~km} \mathrm{~s}^{-1}$. This linear (orthogonal) transformation however yields unphysical $v_{\infty}^{\prime}$-values that differ by $2 \times V_{r}$ for line-of-sight directions in front and behind the primary. For example, the terminal wind velocity in the front hemisphere increases by $V_{r}$ during fastest approach to the observer, while it decreases in the far hemisphere (due to the opposite direction of $v_{x}$ behind the primary). However, $v_{\infty}^{\prime}$ must approach the same velocity in all (radial) directions around the binary system at a longer distance from the stellar surface $\left(r>10 R_{1}\right)$. At a longer distance, the acceleration of the wind mainly results from the primary's strong radiation field. Particles escaping at the wind base will gain mechanical momentum from the orbital motion (measured in the observer's frame), which becomes negligibly small when compared to the radiative driving by the stellar radiation field at a longer distance in the wind.

We compute a wind crossing time $t\left(r_{\mathrm{W}}\right)=\int_{R_{1}}^{r_{\mathrm{w}}} \mathrm{d} r / v(r)$ of $t(10) \simeq 10 \mathrm{~d}$ with $\beta=1$ for particles travelling from $R_{1}$ to $10 R_{1}$. At the latter distance, the wind velocity is $v \simeq 0.9 \times$ $v_{\infty}=900 \mathrm{~km} \mathrm{~s}^{-1}$. The wind particles travel another $\sim 12 \mathrm{~d}$ $(t(30)=22 \mathrm{~d})$ before reaching $30 R_{1}$ in the model where $v \simeq v_{\infty}$. Hence, the dynamical timescale of the wind outflow in our model is sufficiently long compared to $P=60.8 \mathrm{~d}$ for the radiative driving to homogenise $v_{\infty}^{\prime}$ to the same velocity in all directions around the binary system. We therefore adopt a modified $\beta$-law providing equal $v_{\infty}^{\prime}$-values at distances $r>10 R_{1}$. The 3D wind velocity structure is given by

$$
\begin{aligned}
v_{x}^{\prime}(r, \theta, \psi)= & -\left(v_{0}+\left(v_{\infty}^{\prime}(\phi)-v_{0}\right)\left(1-\frac{R_{1}}{r}\right)^{\beta^{\prime}}\right) \\
& \times \sin (\theta) \cos (\psi)+V_{r}(\phi),
\end{aligned}
$$



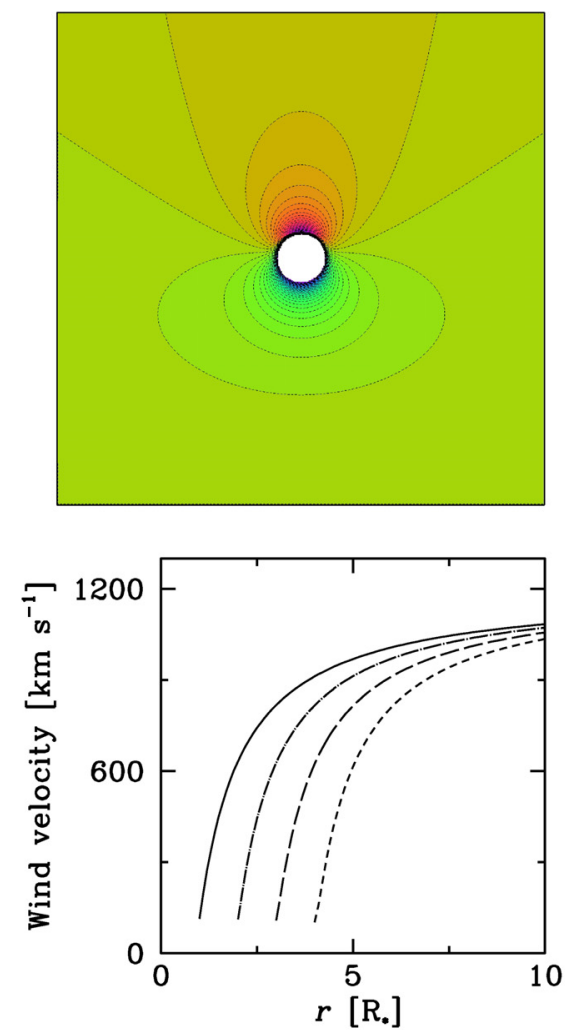
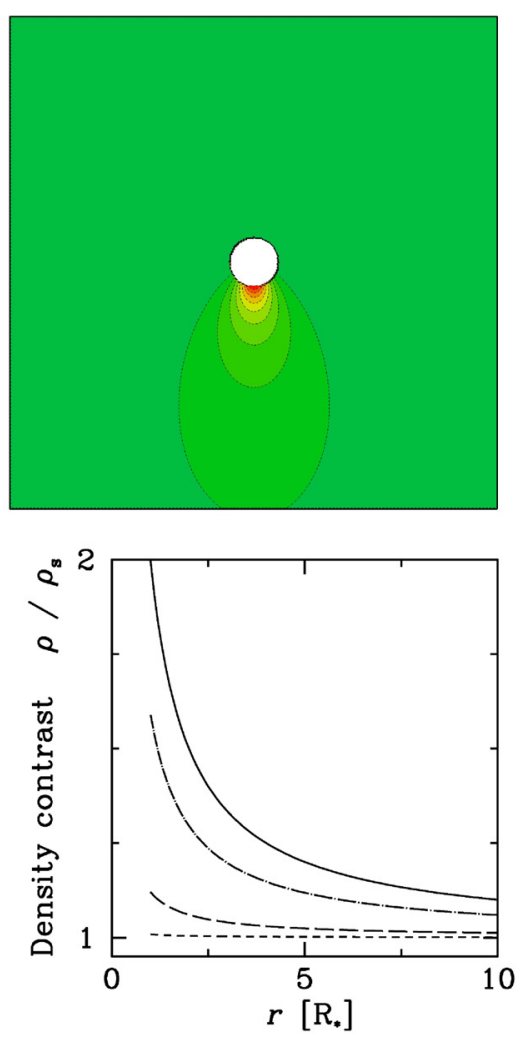

Fig. 22. Top panels: changes in velocity (lefthand panel) and density (right-hand panel) to $10 R_{1}$ in the equatorial plane of the $3 \mathrm{D}$ wind model around the primary star due to orbital motion towards the south. In front of the star (blue colour in online figure) the wind velocity difference in the observer's frame decreases from $V_{r}=-85 \mathrm{~km} \mathrm{~s}^{-1}$ at the surface to $>-10 \mathrm{~km} \mathrm{~s}^{-1}$ for $r>3 R_{1}$. Behind the star (red colour in online figure) it increases from $-85 \mathrm{~km} \mathrm{~s}^{-1}$ to $<10 \mathrm{~km} \mathrm{~s}^{-1}$ for $r>3 R_{1}$. At larger distances from the surface the difference of wind velocity with the stationary wind $\left(V_{r}=0\right)$ model is $<10 \mathrm{~km} \mathrm{~s}^{-1}$ and becomes symmetric in all directions around the binary. Bottom left-hand panel: wind velocity for $\psi=0$ (solid drawn line), $\pi / 3$ (dashdotted line), $2 \pi / 3$ (dashed line), and $\pi$ (shortdashed line). The curves are shifted to the right by $+1 R_{1}$ for clarity. Bottom right-hand panel: radial wind density contrast at these $\psi$ angles for $f=1$ (see Sect. 5.2). where $\theta$ and $\psi$ are the polar and azimuthal angles of a point in the wind at distance $r>R_{1}$ above the stellar surface. We choose the $x$-axis of the model along the line-of-sight to the star. The wind speed at the photosphere $\left(r=R_{1}\right)$ is $v_{0}$. The minus sign of the first term in Eq. (2) follows from the convention for the sign of radial velocity $V_{r}$, depending on the last term on $\phi$. Note that $V_{r}(\phi)=V_{x}^{\text {orb }}(\phi)=V^{\text {orb }}(\phi) \sin (i) \cos (\omega)$, where $V^{\text {orb }}$ is the orbital velocity. Likewise, $V_{y}^{\text {orb }}(\phi)=V^{\text {orb }}(\phi) \sin (i) \sin (\omega)$ and $V_{z}^{\text {orb }}(\phi)=V^{\text {orb }}(\phi) \cos (i)$ are computed with $i, \omega$, and the orbital velocity $V^{\text {orb }}(\phi)$ using the orbital elements in Table 2 . We denote in Eq. (2):

$v_{\infty}^{\prime}(\phi)=v_{\infty}+V_{r}(\phi) \sin (\theta) \cos (\psi)$,

which modifies $v_{\infty}$ in the frame of the primary by the $x$-axis projected radial velocity. Inserting Eq. (3) in Eq. (2) for $r \rightarrow \infty$ yields $v_{x}^{\prime}\left(\theta=\frac{\pi}{2}, \psi=0\right)=-v_{\infty}$ and $v_{x}^{\prime}\left(\theta=\frac{\pi}{2}, \psi=\pi\right)=v_{\infty}$. Hence, the non-linear base transformation provides the same terminal wind speed in front and behind the primary in the frame of the observer by adjusting $v_{\infty}$ for the (projected) orbital velocity at $\phi$. The $v_{y}^{\prime}$ - and $v_{z}^{\prime}$-components are given by:

$$
\begin{aligned}
v_{y}^{\prime}(r, \theta, \psi)= & -\left(v_{0}+\left(v_{\infty}^{\prime}(\phi)-v_{0}\right)\left(1-\frac{R_{1}}{r}\right)^{\beta^{\prime}}\right) \\
& \times \sin (\theta) \sin (\psi)+V_{y}^{\mathrm{orb}}(\phi),
\end{aligned}
$$

and

$v_{z}^{\prime}(r, \theta)=-\left(v_{0}+\left(v_{\infty}^{\prime}(\phi)-v_{0}\right)\left(1-\frac{R_{1}}{r}\right)^{\beta^{\prime}}\right) \cos (\theta)+V_{z}^{\mathrm{orb}}(\phi)$,

where the last term of Eq. (3) is replaced by $V_{y}^{\text {orb }}(\phi) \sin (\theta) \sin (\psi)$ and $V_{z}^{\text {orb }}(\phi) \cos (\theta)$, respectively. We also modify the wind acceleration law in the base transformation. The value of $\beta^{\prime}$ in Eqs. (2), (4), and (5) denotes a small adjustment of $\beta$ required to sustain the same wind acceleration in all directions around the binary. It results from the product of $v_{\infty}^{\prime}$ and $\left(1-R_{1} / r\right)^{\beta^{\prime}}$. If $\beta^{\prime}=\beta$ for constant $\beta$, the multiplication yields slightly variable wind acceleration over $(\theta, \psi)$-directions, because Eq. (2) modifies $v_{\infty}^{\prime}$ with the projected orbital velocity. Using an angle-dependent adjustment for $\beta$ of the form $\beta^{\prime}=\beta+p \sin (\theta) \cos (\psi)$, we compute almost identical wind accelerations in all directions in the observer's rest frame with $p \simeq 0.07$ for $\beta=1, v_{\infty}=1200 \mathrm{~km} \mathrm{~s}^{-1}$, and $\left|V_{r}\right| \leq 100 \mathrm{~km} \mathrm{~s}^{-1}$.

It is important to point out that the 3D binary wind geometry we develop for MWC $314 v^{\prime}=\left(v_{x}^{\prime}, v_{y}^{\prime}, v_{z}^{\prime}\right)$ is not spherically symmetric around the centre of gravity of the binary system. At the base of the wind, the outflow (for inertial observers) is centred around the primary orbiting the binary centre of gravity, while the wind flow at a long distance assumes constant terminal velocity in all directions around the system. The 3D wind geometry is therefore asymmetric in the vicinity of the primary's orbit and directly depends on $\phi$, while the wind flow at a long distance becomes symmetric and independent of $\phi$. The left-hand top panel of Fig. 22 shows the change in wind velocity $v^{\prime}-v$ in the equatorial plane around the primary $(\theta=\pi / 2)$ due to the orbital motion. We compute $v^{\prime}=\sqrt{v_{x}^{\prime 2}+v_{y}^{\prime 2}}$ with Eqs. (2)-(4) for $V_{r}=-85 \mathrm{~km} \mathrm{~s}^{-1}, V_{y}^{\text {orb }}=0 \mathrm{~km} \mathrm{~s}^{-1}, v_{0}=10 \mathrm{~km} \mathrm{~s}^{-1}$, and $p=0.07$. For given $\phi$, the primary moves in the direction towards the observer south in the panel. The wind velocity in the observer's frame $v$ is computed for $V_{r}=0 \mathrm{~km} \mathrm{~s}^{-1}$ and $\beta^{\prime}=\beta=1$ $(p=0)$. In front of the primary, the wind velocity difference in the observer's frame decreases from $-85 \mathrm{~km} \mathrm{~s}^{-1}$ at the surface ( $r=R_{1}$ ) to above $-10 \mathrm{~km} \mathrm{~s}^{-1}$ for $r>3 R_{1}$. Behind the star, it increases from $-85 \mathrm{~km} \mathrm{~s}^{-1}$ at the surface to below $10 \mathrm{~km} \mathrm{~s}^{-1}$ for $r>3 R_{1}$. At longer distances from the surface (shown in Fig. 22 to $r=10 R_{1}$ ), the difference of the radial wind velocity and the stationary wind (when the primary were static for the observer) is less than $10 \mathrm{~km} \mathrm{~s}^{-1}$ in all directions around the star. The bottom left-hand panel of Fig. 22 shows $v^{\prime}(r)$ for $\psi=0, \pi / 3$, 
$2 \pi / 3$, and $\pi$. For clarity, the four curves are shifted to the right by $+1 R_{1}$. The dashed lines in the upper panel of Fig. 22 mark contours of equal $v^{\prime}-v$ that are denser in front of the primary than behind it. It results from the orbital velocity, which slightly increases the radial wind velocity gradient in the direction of the star's orbital motion, while decreasing it in the opposite direction. The influence of orbital velocity on the overall wind velocity structure, however, rapidly becomes negligibly small $\left(<10 \mathrm{~km} \mathrm{~s}^{-1}\right)$ due to the asymmetric base transformation presented. It allows us to adequately measure the effect of (small) $\phi$-dependent density enhancements in the wind of the primary using 3-D RT modelling of selected spectral lines.

\subsection{Wind model density structure}

We parametrise the wind density structure around the primary of MWC 314 with

$$
\begin{aligned}
\rho(r, \theta, \psi)= & \rho_{\mathrm{s}}(r)\left(1+\frac{f}{r} \exp \left(-\left(\frac{\psi-\Upsilon_{0}}{\varsigma_{0}}\right)^{2}\right)\right. \\
& \left.\times \exp \left(-\left(\frac{\theta-\Theta_{0}}{\vartheta_{0}}\right)^{2}\right)\right),
\end{aligned}
$$

where $\rho_{\mathrm{s}}=\rho_{0} / r^{2}$ is the smooth wind density and $f \geq 0$ a free parameter that increases $\rho_{\mathrm{s}}$ in the $\left(\Theta_{0}, \Upsilon_{0}\right)$-direction. We adopt the $r^{-3}$-dependence for the increase in wind density, hence $\rho \rightarrow \rho_{\mathrm{s}}$ for $r \rightarrow \infty$. The constant values (in units of angle) $\varsigma_{0}$ and $\vartheta_{0}$ determine the azimuthal and polar density distributions around the primary star. Smaller values provide wind density structures that are more asymmetric and oriented towards the $\left(\Theta_{0}, \Upsilon_{0}\right)$ direction. The top right-hand panel of Fig. 22 shows the density contrast $\rho / \rho_{\mathrm{s}}$ in the equatorial plane $(\theta=\pi / 2)$ to $r=10 R_{1}$. We set $\Upsilon_{0}=0$ and $\Theta_{0}=\pi / 2$, providing enhanced wind density in front of the star moving south. We set the azimuthal density distribution to $\varsigma_{0}=\pi / 4$, which is equivalent to a mean opening angle of $\sim 2 \varsigma_{0}=\pi / 2$ for the larger density contrast in the wind. The bottom right-hand panel plots $\rho / \rho_{\mathrm{s}}$ for $\psi=0$ (solid drawn line), $\pi / 3$ (dash-dotted line), $2 \pi / 3$ (dashed line), and $\pi$ (short-dashed line). For $f=1$, the wind density increases by $100 \%$ at the surface in front of the star but rapidly decreases to below $50 \%$ at $r=2 R_{1}$ and $<10 \%$ for $r>10 R_{1}$. The percentages evidently depend on the $f / r$-factor adopted in Eq. (6). The RT modelling presented in Sect. 5.3 shows that the latter factor, combined with the polar and azimuthal Gaussian distributions in Eq. (6), adequately parametrizes the large-scale asymmetric geometry of wind density in MWC 314.

\section{3. $3 D$ radiative transfer modelling}

The WIND3D code calculates the transport of radiation in spectral lines in a 3D Cartesian grid. The code solves the line transfer problem for arbitrary 3D wind velocity $v_{i j k}(r, \theta, \psi)$ - and density $\rho_{i j k}(r, \theta, \psi)$-structures around a spherical source radiating continuum flux. The line source function iterations employ an accelerated exact lambda iteration scheme for computing the mean intensities $\bar{J}_{i j k}$ in $101^{3}$ gridpoints. The RT scheme is fully parallelized with excellent load-balancing. We compute the detailed profile of He I $\lambda 5876$ in two steps using 36 CPU cores on the ROB's central compute server "Plato". It addresses 192 64-bit SGI shared-memory cores at $1.7 \mathrm{GHz}$ with batch queue processing. First, the 3-D line source function is converged starting from a Sobolev approximation to local intensity fluctuations below $1 \%$. The source function iterations are the most time-consuming, because they require integration over 80 angles to account for random line opacity- and velocitydistributions in the 3D wind model that irradiates each gridpoint from all directions. Convergence can considerably slow down in non-uniform $v$ - and $\rho$-structures, although we implement the Cartesian upwind discretisation method (Adam 1990) to avoid non-convergence. This finite element method is unconditionally stable but requires large memory allocations. Since the wind model is symmetric about the orbital plane, we initially compute the source function above the plane, followed by a fast convergence test for all gridpoints below the plane. This about halves the computation time of the first step. The second step interpolates the source function on a finer grid of $801^{3}$ points and calculates the transfer equation for user-defined radial lines of sight around the model. It integrates the specific intensities over the front plane perpendicular to the line(s) of sight, hence providing the 3D emergent line fluxes.

WIND3D solves the non-LTE transfer problem in scatteringdominated spectral lines formed in the extended winds of massive stars. We compute the dynamic He I line profile for 60 equal time intervals in $0 \leq \phi<1$ over the orbit of the primary. This corresponds to uniform steps of $6^{\circ}$ for 60 directions around the star observed in orbit at a constant inclination angle of $i=72.8^{\circ}$. The detailed line profile is computed at 100 wavelength points between $-1300 \mathrm{~km} \mathrm{~s}^{-1}$ and $1300 \mathrm{~km} \mathrm{~s}^{-1}$. Note that the computation time per line profile is considerably shorter ( $\sim 10 \mathrm{~min})$ than for the line source function iterations of $\sim 3-5 \mathrm{~h}$. The dynamic spectrum calculations require total computation times of $\sim 9-11 \mathrm{~h}$ per input model (using 36 cores), which is still acceptable for exploring a broad range of wind model parameters. Finer meshes require more CPU time and even larger memory allocation. The grid resolution of $101^{3}$ points covers $\pm 30 R_{1}$, which is adequate for modelling the effects of small velocity perturbations and density enhancements in the parametrized binary wind model developed in Sects. 5.1 and 5.2. Similar grid resolutions of $71^{3}$ were used in Lobel \& Blomme (2008) for the detailed modelling of discrete absorption components observed in $\mathrm{P}$ Cyg profiles of luminous hot stars with 3D hydrodynamic wind models that incorporate co-rotating interaction regions.

We compute the He I $\lambda 5876$ line opacity $\chi_{i j k}^{l}$ discussed in Appendix B of Lobel \& Blomme (2008) with the parametrized binary wind model of $\rho_{i j k}$ and $v_{i j k}$. We use the smooth wind opacity parameters $\alpha_{1}=0.1, \alpha_{2}=0.8$, and $T_{\text {tot }}=0.18$. The smooth wind acceleration parameters are $\beta=1, v_{\infty}=1200 \mathrm{~km} \mathrm{~s}^{-1}$, and $v_{0}=10 \mathrm{~km} \mathrm{~s}^{-1}$. The thermal line broadening in the Gaussian line profile function is $v_{\text {th }}=11 \mathrm{~km} \mathrm{~s}^{-1}$, which is appropriate for kinetic gas temperatures at $\sim 20 \mathrm{kK}$ in the absorption line formation region of unsaturated $\mathrm{He}$ I lines. The He I $\lambda 5876$ line is a $D_{3}$ transition between triplet states $1 \mathrm{~s} 2 \mathrm{p}^{3} \mathrm{P}^{\circ}-1 \mathrm{~s} 3 \mathrm{~d}^{3} \mathrm{D}$ having $\chi_{\text {low }}=20.9 \mathrm{eV}$, which is comparable to the Si II triplet modelled in Sect. 4.2 with 1D RT in the stellar photosphere. WIND3D computes the 3D RT in the wind in the 2-level atom approximation. The $T_{\text {tot }}$ parameter determines the total wind line opacity, depending on the line oscillator strength and the He abundance in the wind. The projected (micro-)turbulent velocity in the profile function is $\zeta_{\mu}=5 \mathrm{~km} \mathrm{~s}^{-1}$ (see Sect. 4.2). We compute the 3-D structured wind velocity with Eqs. (2)-(5) using the best fit RV-curve $V_{r}(\phi)$ for the $16 \mathrm{RV}$ observations (upper panel of Fig. 5). We model the large-scale 3D wind density around the primary with Eq. (6) for $f$-values between 1 and 10 . We set $\Upsilon_{0}(\phi)=2 \pi \phi+\phi_{0}$ and $\Theta_{0}=\pi / 2$. Hence, the density enhancement in the wind model for $\phi_{0}=0$ is always oriented with $\phi$ towards the leading side of the primary's orbit in its orbital plane.

The left-hand top panel of Fig. 23 shows the dynamic spectrum of He I computed for $f=10$ and $\varsigma_{0}=\vartheta_{0}=\pi / 4$. The 


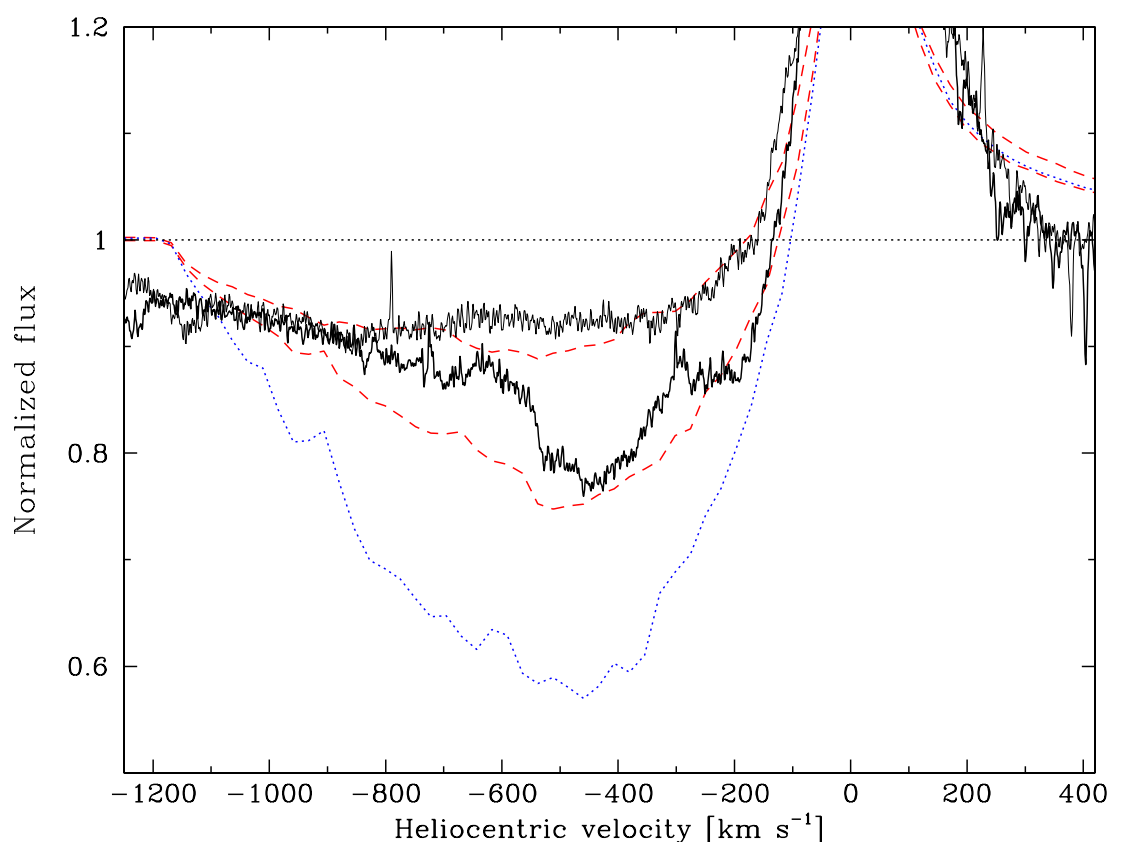

Fig. 24. Detailed 3D RT modelling of the orbitally modulated $\mathrm{He}$ I $\lambda 5876$ line we observe in MWC 314 at $\phi=0.444$ (thin drawn solid line) and at $\phi=0.795$ (boldly drawn solid line). The best fit to the P Cyg absorption at both orbital phases (dashed lines) requires an asymmetric distribution of wind density around the primary star with the density enhancement factor $f=3.3$ in Eq. (6). The absorption line computed with the model of $f=10$ is too strong at $\phi=0.795$ (dotted line). absorption portion of the P Cyg profile is variable and strengthens for $\phi=0.55-0.95$. The increase in absorption results from the wind density enhancement in the model in our line of sight to the primary star. During these orbital phases, the line opacity exceeds the smooth wind opacity by a factor of $\rho / \rho_{\mathrm{s}}$. The value of $\varsigma_{0}$ determines the duration of higher absorption, while $\phi_{0}$ sets the orbital phase when the increase occurs. The lower panels of Fig. 23 show the normalised line fluxes at 12 orbital phases shifted upwards between 0 and 1 . For comparison, the middle panel shows the dynamic spectrum computed with $\varsigma_{0}=\vartheta_{0}=$ $\pi / 3$. The increase in line absorption occurs for $\phi=0.45-1.0$ due to the increased opening angle for higher wind density around the star. We also increase the $V_{r}$-amplitude by a factor of four to amplify the effects of orbital motion on the emergent line fluxes. The primary's orbital velocity causes periodical Doppler shifts in portions of the line profile with the radial velocity forming near the wind base. The S-wave becomes clearly noticeable in the red-shifted emission line wing. On the other hand, the blueshifted absorption around $v \simeq 1200 \mathrm{~km} \mathrm{~s}^{-1}$ is stationary due to the constant $v_{\infty}$ in our wind model in all directions around the binary system (see Sect. 5.1).

The right-hand panels of Fig. 23 show the best fit dynamic spectrum to the He I $\lambda 5876$ line we observe in MWC 314. The parameter values of $f=3.3$ and $\varsigma_{0}=\vartheta_{0}=\pi / 4$ provide the best fit of the absorption variability with $\phi$ in the P Cyg profile. The absorption line variability computed explains the periodic changes observed with $\phi$ in the He I lines of Fig. 12. Figure 24 shows the detailed comparison of observed and theoretical He I 15876 line profiles. The solid drawn lines are spectra observed at $\phi=0.444$ (thin drawn line) and at $\phi=0.795$ (thick line). The theoretical spectra are overplotted with dashed lines. At $\phi=0.795$, the He I absorption increases by $\sim 15 \%$ due to the increase of wind density in our line of sight by a factor of $\sim 3.3$ for distances of $r<2 R_{1}$ in the wind of the primary. It causes a variable He I $\lambda 5876$ line opacity in wind regions of $v<600 \mathrm{~km} \mathrm{~s}^{-1}$ due to the orbitally modulated orientation of enhanced wind density in front of the supergiant. For example, the higher wind density leading the primary's orbit for $\phi=0.1-0.5$ is partially blocked from view by the star's visible surface hence diminishing the line opacity contributions to the
He I absorption. During these orbital phases, the absorption at low wind velocity also Doppler shifts to longer wavelengths and becomes more filled in by the He I emission line, yielding the decrease to weaker blue-shifted line absorption. The He I absorption computed for $f=10$ is significantly too strong at $\phi=0.795$ (dotted line). In the modelling, we tested $\beta$-wind values between 1 and 3 . We compute a slope in the violet He I emission line wing considerably too shallow below $200 \mathrm{~km} \mathrm{~s}^{-1}$ for $\beta \geq$ 1.5. $\beta=1$ yields the best fit to the He I line wing, providing a wind velocity structure with sufficiently fast acceleration to $v_{\infty}=1200 \mathrm{~km} \mathrm{~s}^{-1}$.

We emphasise that the $f$-factor determined in Eq. (6) for an asymmetric geometry of wind density around the primary star of MWC 314 is limited to 3.3 using 3D RT modelling of orbitally modulated He I P Cyg profiles. At a distance of $1 R_{1}$ above the stellar surface $\left(r=2 R_{1}\right)$, the highest wind density in the direction of $\psi=\Upsilon_{0}$ is $\rho(r=2)=\rho_{0}(1+3.3 / 2) / 4 \simeq 2 \rho_{0} / 3$ for $\rho_{\mathrm{s}}(r)=\rho_{0} / r^{2}$ and $\rho_{0}$ the density at the wind base of $r=1 R_{1}$. Hence, we find that the wind density in front of the primary rapidly decreases to below $\sim \rho_{0}$ within $1 R_{1}$ above the stellar surface. The asymmetric geometry of wind density computed is therefore limited to a volume around the primary within the periastron distance. It indicates that the primary's orbital motion, besides periodically modulating the He I $\lambda 5876$ line formation region, also produces the asymmetric geometry of wind density around the supergiant that can result from its appreciable surface deformation from a sphere (see Sect. 4.1). This directional increase of wind density towards the front side of the primary's orbital path can also support a wind accretion model in MWC 314 due to enhanced wind mass-loss (partly), forming a circumbinary disc in the system's orbital plane.

\section{Discussion}

MWC 314 is a single-lined spectroscopic binary with a luminous early B-type supergiant. Its overall wind and SED properties are very comparable to that of LBVs, such as P Cyg. This is not surprising, given the co-location of MWC 314 with other LBVs in the H-R diagram, as shown in Fig. 25. MWC 314 has an 


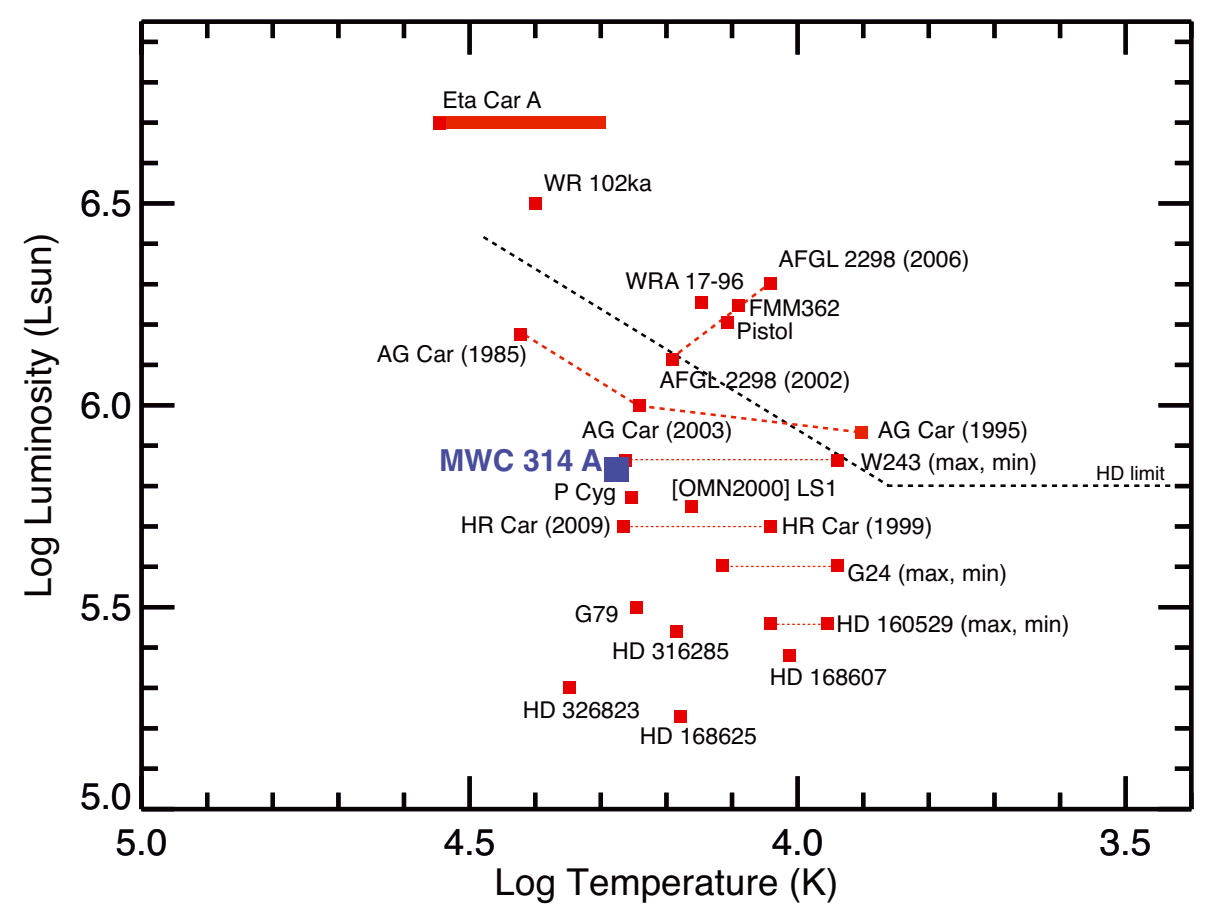

Fig. 25. Hertzprung-Russell diagram showing the location of the primary star in MWC 314 (labelled MWC 314 A) and other selected LBVs. The sources of the stellar parameters of the LBVs are compiled by Groh et al. (2013). average luminosity of an LBV, which is very similar to P Cygni and W243 (Ritchie et al. 2009). MWC 314 is surrounded by a symmetric inner $\mathrm{H} \alpha$ nebula to $20^{\prime \prime}$, however showing a distinct bipolar shape farther out. Some important aspects of the spectroscopic monitoring and modelling of the binary system in this paper deserve a more careful discussion.

We propose that the primary star of MWC 314 is an LBV in a dormant state. It is very similar to the cLBV RMC 81 that has $\mathrm{H}$ Balmer and Paschen series lines showing strong P Cyg profiles. Wolf et al. (2000) present a "working" model of RMC 81 (see their Fig. 7) with an interaction region around the periastron passage between the primary and the unseen companion. Massey (2006) remarks however that the optical spectrum of RMC 81 contains He I lines without a strong emission component. The He I $\lambda 7065$ line in RMC 81 shows weak emission. We observe considerably stronger He I $\lambda 7065$ emission in MWC 314 with normalised emission maxima $~ 3-4$ times above the continuum flux level, which is comparable to the line in P Cyg. Its He I profiles are almost identical to the P Cyg profiles with weak blue-shifted absorption that is observed in the LBV He 3-591 by Smith et al. (1994, see their Figs. 2b and 8). P Cyg and He 3-519 are surrounded by nearly spherical nebulae found in other LBVs, although both stars currently lack evidence of a close binary system. Gvaramadze et al. (2010) report IR observations of an arc-like nebula attached to RMC 81. It is also interesting that Hofmann et al. (2002) favour the binary hypothesis for MWC 349A to explain very strong H Balmer (and He I) emission and a non-spherical distribution of circumstellar matter. MWC 349A shows a clear bipolar nebula and is surrounded by a circumstellar disc observed almost edgeon. Its spectral properties are consistent with a $\mathrm{B}[\mathrm{e}]$ supergiant, such as the strong He I emission lines that we also observe in MWC 314. Gvaramadze \& Menten (2012) have recently suggested that MWC 349A is likely to be an LBV. It prompts us to ask the question if the extended bipolar nebula of MWC 314 has been produced by the close binary system. A binary-driven wind model shaping the bipolar geometry of the material ejected from the system during outbursts appears to be a possible scenario. We note that the companion could plow through the outer layers of the primary if the primary star develops $\mathrm{S}$ Dor variability (i.e., a rapid increase of $R_{1}$ ). This scenario has been advocated by Kashi \& Soker (2009) to explain the Giant Eruption of $\eta$ Car. Groh et al. (2009b) presented an interesting analysis of these radius changes in the LBV AG Car related to changes of $L_{\star}$ in 2001-2003.

The unequal $V$ brightness minima observed in MWC 314 are due to an eclipsing binary with a relatively small size of the primary's orbit $(\simeq 1 \mathrm{AU})$. We observe a semi-detached binary system with the primary star that fills its critical Roche lobe and strong tidal interactions that deform the stellar surface from the spherical shape. Our 3D RT modelling reveals asymmetric wind outflow of increased wind density in front of the primary's orbit, which modulates the He I P Cyg absorption line portions around $\phi=0.65-0.85$. The $3 \mathrm{D}$ wind geometry computed is likely more complicated due to important effects of tidal and orbital motion around an inner wind region where strong mass-loss can accrete and accumulate in an extended circumbinary disc. The Coriolis force and the radiative wind driving in the orbital plane would direct the wind flow of increased density from the primary away from the line through $L_{1}$ (connecting the stellar centres) towards the region leading the primary's orbital path. The transfer of wind mass towards the circumbinary disc efficiently converts wind momentum to angular momentum at an inner disc radius around the primary's periastron distance. There are two important findings in our analysis of MWC 314 related to this. First, the projected rotational velocity $V_{\text {rot }} \sin i \simeq 50 \mathrm{~km} \mathrm{~s}^{-1}$ in photospheric absorption lines (see Sect. 4.2) is smaller or comparable to the maximum kinematic velocity that we observe in prominent emission lines emerging from the circumbinary disc. The largest HWHM peak separation of double-peaked $\mathrm{H}$ Paschen emission lines is $\sim 65 \mathrm{~km} \mathrm{~s}^{-1}$ (see Table 4). The primary's (low) rotation rate is therefore synchronous with the disc rotation rate at the inner boundary where wind mass is accreted. The nearly synchronous rates can result from the steady transfer of wind mass to the disc possibly due to critical Roche overflow at $L_{1}$. Next, we can estimate the amount of disc accretion from stellar winds with Bondi-Holye-Lyttleton accretion (Edgar 2004). Literature generally provides the BHL accretion rate of wind mass from $m_{1}$ 
into a disc around $m_{2}$ by

$\dot{m}_{\mathrm{d}}=\frac{4 \pi G^{2} m_{2}^{2} \rho_{\mathrm{a}}}{v_{\mathrm{a}}}$,

where $v_{\mathrm{a}}$ is the wind velocity at the accretion radius $r_{\mathrm{a}}=$ $2 G m_{2} / v_{\mathrm{a}}^{2}$ (Bondi 1952). Wind outflow from $m_{1}$ is captured within $r_{\mathrm{a}}$ around $m_{2}$. We use $r_{\mathrm{a}} \simeq a_{1}$ with $a_{1} \simeq R_{1}$ in MWC 314 . Hence, $a_{1}$ is the distance $r=2 R_{1}$ from the centre of $m_{1}$, where the maximum wind density $\rho_{\mathrm{a}}(r=2) \simeq 2 \rho_{0} / 3$ for $f=3.3$ in Eq. (6). The radiatively driven wind velocity is $v_{\mathrm{a}}(r=2)=$ $v_{0}+v_{\infty} / 2^{\beta}$, or $v_{\mathrm{a}}=v_{\infty} / 2$, since $v_{0} \ll v_{\infty}$ and $\beta=1$. The wind mass-loss rate at the surface of $m_{1}$ is $\dot{m}_{1}=4 \pi R_{1}^{2} \rho_{0} v_{0}$, which yields with Eq. (7):

$\dot{m}_{\mathrm{d}}=\frac{16}{3} \frac{G^{2} m_{2}^{2}}{R_{1}^{2} v_{\infty}^{3} v_{0}} \dot{m}_{1}$.

We calculate $\dot{m}_{\mathrm{d}}=1.03 \times \dot{m}_{1}$ for $m_{2}=26.26 M_{\odot}, R_{1}=86.8 R_{\odot}$ (Table 2), $v_{\infty}=1200 \mathrm{~km} \mathrm{~s}^{-1}$, and $v_{0}=10 \mathrm{~km} \mathrm{~s}^{-1}$. The factor in front of $\dot{m}_{1}$ in Eq. (8) scales with $m_{2}^{2}$ and ranges from 0.95 to 1.40 for $25.3 M_{\odot} \leq m_{2} \leq 30.7 M_{\odot}$. We estimate that the disc accretion rate in MWC 314 is about the same order of magnitude as the primary's wind mass-loss rate $\dot{m}_{1}$. Our estimate of $\dot{m}_{\mathrm{d}}$ reveals that a massive companion star in MWC 314 can accumulate a considerable fraction of the wind mass shed by the primary star by disc accretion. For a typical LBV, smooth wind mass-loss rates are of $\sim 10^{-5} M_{\odot} \mathrm{yr}^{-1}$, this implies that $\sim 1 M_{\odot}$ can amass in the circumbinary disc during the LBV evolutionary phase of $\sim 10^{5} \mathrm{yr}$. This mass-loss scenario yields a massive binary system supporting a large (and heavy) circumbinary disc. The inner boundary becomes dynamically more unstable with time due to strong tidal perturbations by the massive companion stars. A large amount of angular momentum stored in the disc would escape the binary during unstable events that disrupt the inner boundary structure on much shorter timescales. We think therefore that a break-up mechanism of the inner disc boundary can cause short-lived outburst events with very strong mass-loss from MWC 314. Such an outburst may have produced the large bipolar nebulae observed around MWC 314. Note that we compute a fraction $\dot{m}_{\mathrm{d}} / \dot{m}_{1}$ exceeding 0.1 with Eq. (8). Over longer periods of $2-510^{5} \mathrm{yr}$, at least 0.2 to $1 \times M_{\odot}$ is stored in the disc, which considerably increases the total disc mass, thereby rendering it dynamically unstable in the massive binary system. This may cause recurring outburst events during which disc mass becomes partly ejected from the system, or drive punctuated events with very high mass-loss rates. BHL disc accretion provides an interesting mechanism for expelling accumulated wind material very far from a massive binary system in (bipolar) directional outflow.

One may also wonder what the evolutionary stage of MWC 314 currently is and what would be its ultimate fate. Is the massive primary star perhaps on the verge of core-collapse? If so, one would expect that the supernova shock wave would interact with the potentially massive H-rich circumstellar disc around MWC 314. In this case, a SN IIn would occur, or namely, a SN with narrow $\mathrm{H}$ lines in the spectrum. Perhaps MWC 314 is in an earlier stage of evolution of systems like SN 2009ip, which has shown multiple outbursts and possibly has gone under core-collapse (Pastorello et al. 2013). It is also interesting to note that Levesque et al. (2012) find evidence of a circumstellar disc in SN 2009ip, resembling the geometry that we found in MWC 314. Alternatively, the primary of MWC 314 is perhaps still far from core collapse. In that case, it will eventually lose its entire $\mathrm{H}$ envelope, becoming a W-R star and exploding as a SN Ibc.

\section{Concluding remarks}

We summarise the results for MWC 314 as follows:

1. MWC 314 is a massive binary with $P=60.8 \mathrm{~d}$ and $e=0.23$. The orbital elements are accurately determined from best fits of the combined radial velocity and $V$ curves. We observe the system for $i=72.8^{\circ} \pm 13^{\circ}$. The maximum separation of both stars is $1.22 \mathrm{AU}$.

2. The primary star is a hot supergiant with $T_{\text {eff }}=18 \mathrm{kK}$ and $30.7 M_{\odot} \leq m_{1} \leq 44.2 M_{\odot}$. It is a slowly rotating massive supergiant with $V_{\text {rot }} \sin i \simeq 50 \mathrm{~km} \mathrm{~s}^{-1}$ and $R_{1}=86.8 R_{\odot}$ that fills it Roche volume in a semi-detached binary system.

3. Long-term high-resolution spectroscopic monitoring during 2 yr reveals that the optical emission line spectrum is orbitally modulated. The $\mathrm{H}$ Balmer and Paschen emission lines, the He I P Cyg line profiles, and the double-peaked permitted metal emission lines all show variability linked to the orbital period.

4. A detailed comparison of the SEDs of MWC 314 and LBV P Cyg reveal nearly identical IR slopes for free-free emission with wavelength. It signals a radiatively accelerating wind in MWC 314 with a mean $r^{-2}$ density structure of the smooth wind, which is similar to P Cyg.

5. We develop a modified $\beta$-law for modelling the large-scale wind velocity (and density) structure for a massive star observed in a binary orbit. The 3D wind velocity structure accounts for the orbital motion in a radiatively driven wind with constant terminal velocity in all directions around the binary system.

6. Detailed 3D radiative transfer modelling of He I P Cyg line profiles with orbital phases reveals that the geometry of wind density around the primary is asymmetric. It causes orbitally modulated variability of P Cyg absorption that forms in the primary's extended wind. We observe wind outflow above $1000 \mathrm{~km} \mathrm{~s}^{-1}$ in He I $\lambda 5876$. The RT modelling requires a density enhancement factor of 3.3 above the smooth wind density. The asymmetric increase of wind density leads the orbital path of the primary.

7. The double-peaked emission lines reveal a significant decrease in peak velocity separation from $\sim 65 \mathrm{~km} \mathrm{~s}^{-1}$ in $\mathrm{H}$ Paschen, $\sim 55 \mathrm{~km} \mathrm{~s}^{-1}$ in Fe II, and $\sim 25 \mathrm{~km} \mathrm{~s}^{-1}$ in $[\mathrm{N}$ II] lines. It can signal a Keplerian rotating disc in MWC 314 with lower rotation velocities in emission line formation regions farther from the binary centre of gravity.

8. We do not observe He II lines in MWC 314. Radiative transfer modelling of the IR Spitzer spectrum rules out the spectral O-type for the secondary star. The radial velocity and brightness curves indicate a less-luminous cool giant of $25.3 M_{\odot} \leq m_{2} \leq 30.7 M_{\odot}$. We estimate a wind accretion rate for the circumbinary disc of this massive binary system around the primary's wind mass-loss rate.

9. We observe variable continuum normalised maximum fluxes in strong $\mathrm{H} \alpha$ and $\mathrm{H} \beta$ emission lines that clearly correlate to the orbital phases. The $V$-brightness curve is due to an eclipsing binary system with periodical $V$-continuum flux changes that cause $\mathrm{H} \alpha$ and $\mathrm{H} \beta$ line variability. Strong $\mathrm{H}$ Balmer emission emerges from an inner $\mathrm{H} \alpha$ nebulae that we observe symmetrically around the binary to $\sim 20^{\prime \prime}$. At larger angular distances, weaker $\mathrm{H} \alpha$ emission emerges from a bipolar nebula that was previously observed around MWC 314.

Long-term spectroscopic monitoring shows that MWC 314 is an excellent target for studying the physics of winds in massive binaries. The binary period of only $\simeq 2 \mathrm{~m}$ and $e=0.23$ offers a unique laboratory for investigating orbitally modulated 
spectral variability in one of the most massive hot stars. Our asymmetric wind model of MWC 314 contributes to the ongoing efforts for unraveling the driving mechanisms of LBV winds with punctuated mass-loss. An important question is how these outburst events can eject large amounts of hydrogen gas and shape structured nebulae around LBVs before they evolve to the Wolf- Rayet phase. It also poses the more fundamental question whether binarity or multiplicity is a prerequisite for $\mathrm{S}$ Dor and Hubble-Sandage stars. The system $\eta$ Car is a long-period $(\sim 5.5 \mathrm{yr})$ massive binary with a compact and, possibly, massive companion (i.e., Damineli et al. 2000). The system HD 5980 has a massive $\mathrm{W}-\mathrm{R}$ companion star with an orbital period of $\sim 20 \mathrm{~d}$. We find that the secondary of MWC 314 is also a massive star with an orbital period $\sim 3$ times longer. The number of known binary hypergiants, showing the orbitally modulated P Cyg line variability that we observe in MWC 314, is however still very limited. We are in the course of spectroscopically monitoring other LBVs and cLBVs, to search for similar line profile variability in examples, such as Cyg OB2 12, HD 168607, the Pistol star, and P Cygni. The large-scale 3-D wind geometry in massive binary systems should be further explored and compared to detailed radiative transfer modelling of high-resolution spectroscopic observations.

Acknowledgements. A.L. acknowledges funding from the ESA/Belgian Federal Science Policy in the framework of the PRODEX programme. The HERMES project and team acknowledge support from the Fund for Scientific Research of Flanders (FWO), Belgium, support from the Research Council of K.U. Leuven (Belgium), support from the Fonds National de la Recherche Scientifique, Belgium (FNRS), from the Royal Observatory of Belgium and from the Landessternwarte Tautenburg (Germany). J.H.G. is supported by an Ambizione Fellowship of the Swiss National Science Foundation. We thank Drs. N. Gorlova and N. Cox of Univ. of Leuven, Belgium, for Mercator-MEROPE observations of MWC 314 in March 2011. We thank the referee for helping to improve the presentation of the paper.

\section{References}

Adam, J. 1990, A\&A, 240, 541

Adelman-McCarthy, J. K., Agüeros, M. A., Allam, S. S., et al. 2008, ApJS, 175, 297

Ardila, D. R., Van Dyk, S. D., Makowiecki, W., et al. 2010, ApJS, 191, 301 Bondi, H. 1952, MNRAS, 112, 195

Brandner, W., Grebel, E. K., Chu, Y.-H., \& Weis, K. 1997, ApJ, 475, L45

Carlsson, M. 1986, Uppsala Astronomical Observatory Reports, 33

Castelli, F., \& Kurucz, R. L. 2013, IAU Symp. 210, eds. N. Piskunov et al., A20

Clark, J. S., Najarro, F., Negueruela, I., et al. 2012, A\&A, 541, A145

Code, A. D., Holm, A. V., \& Bottemiller, R. L. 1980, ApJS, 43, 501

Cutri, R. M., et al. 2012, VizieR Online Data Catalog, II/311

Cutri, R. M., Skrutskie, M. F., van Dyk, S., et al. 2003, VizieR Online Data Catalog, II/246

Damineli, A., Kaufer, A., Wolf, B., et al. 2000, ApJ, 528, L101

Damineli, A., Hillier, D. J., Corcoran, M. F., et al. 2008, MNRAS, 386, 2330

de Jager, C. 2001, in P Cygni 2000: 400 Years of Progress, ASP Conf. Ser., 233, 215

Di Francesco, J., Johnstone, D., Kirk, H., MacKenzie, T., \& Ledwosinska, E. 2008, ApJS, 175, 277

Ducati, J. R. 2002, VizieR Online Data Catalog, II/237

Edgar, R. 2004, New Astron. Rev., 48, 843

Egan, M. P., Price, S. D., Kraemer, K. E., et al. 2003, VizieR Online Data Catalog, V/114

Foellmi, C., Koenigsberger, G., Georgiev, L., et al. 2008, Rev. Mex. Astron. Astrofis., 44, 3

Friend, D. B., \& Castor, J. I. 1982, ApJ, 261, 293

Georgiev, L., Koenigsberger, G., Hillier, D. J., et al. 2011, AJ, 142, 191

Groh, J. H., Damineli, A., \& Jablonski, F. 2007, A\&A, 465, 993

Groh, J. H., Damineli, A., Hillier, D. J., et al. 2009a, ApJ, 705, L25

Groh, J. H., Hillier, D. J., Damineli, A., et al. 2009b, ApJ, 698, 1698
Groh, J. H., Meynet, G., Georgy, C., \& Ekström, S. 2013, A\&A, submitted Gvaramadze, V. V., \& Menten, K. M. 2012, A\&A, 541, A7

Gvaramadze, V. V., Kroupa, P., \& Pflamm-Altenburg, J. 2010, A\&A, 519, A33

Hartman, H., Schef, P., Lundin, P., et al. 2005, MNRAS, 361, 206

Hauck, B., \& Mermilliod, M. 1998, A\&AS, 129, 431

Helou, G., \& Walker, D. W. 1988, Infrared astronomical satellite (IRAS) catalogs and atlases. The small scale structure catalog, 7, 1

Hoffman, M., Stift, M. J., \& Moffat, A. F. J. 1978, PASP, 90, 101

Hofmann, K.-H., Balega, Y., Ikhsanov, N. R., Miroshnichenko, A. S., \& Weigelt, G. 2002, A\&A, 395, 891

Høg, E., Fabricius, C., Makarov, V. V., et al. 2000, A\&A, 355, L27

Humphreys, R. M., \& Davidson, K. 1994, PASP, 106, 1025

Humphries, C. M., Jamar, C., Malaise, D., \& Wroe, H. 1976, A\&A, 49, 389

IPAC. 2008, Galactic Legacy Infrared Mid-Plane Survey Extraordinaire (GLIMPSE) Source Catalog (I + II + 3D), Spitzer Science Center, Caltech, USA

Ishihara, D., Onaka, T., Kataza, H., et al. 2010, A\&A, 514, A1

Kashi, A., \& Soker, N. 2009, New Astron., 14, 11

Kaufer, A., Stahl, O., Tubbesing, S., et al. 1999, The Messenger, 95, 8

Kharchenko, N. V. 2001, Kinematika i Fizika Nebesnykh Tel, 17, 409

Kurchakov, A. V., \& Rspaev, F. K. 2005, Astron. Astrophys. Trans., 24, 317

Lamers, H. J. G. L. M., \& Cassinelli, J. P. 1999, Introduction to Stellar Winds

Le Guillou, L., Davignon, G., Van Winckel, H., et al. 2008, Tech. rep., MERCATOR/MEROPE Reference Manual, University of Leuven, Belgium

Levesque, E. M., Stringfellow, G. S., Ginsburg, A. G., Bally, J., \& Keeney, B. A 2012, ApJ, submitted [arXiv: 1211.4577 ]

Lobel, A., \& Blomme, R. 2008, ApJ, 678, 408

Lobel, A., de Jager, K., \& Nieuwenhuijzen, H. 2013, in 370 Years of Astronomy in Utrecht, eds. G. Pugliese, A. de Koter, \& M. Wijburg, ASP Conf. Ser., 470, 167

Lobel, A., Groh, J., Torres Dozinel, K., et al. 2012, in Proc. Scientific Meeting in Honor of Anthony F. J. Moffat, eds. L. Drissen, C. Rubert, N. St-Louis, \& A. F. J. Moffat, ASP Conf. Ser., 465, 358

Madura, T. I., Gull, T. R., Owocki, S. P., et al. 2012, MNRAS, 420, 2064

Marston, A. P., \& McCollum, B. 2008, A\&A, 477, 193

Martayan, C., Lobel, A., Baade, D., et al. 2012, in Circumstellar Dynamics at High Resolution, eds. A. C. Carciofi, \& T. Rivinius, ASP Conf. Ser., 464, 293

Massey, P. 2006, ApJ, 638, L93

Mermilliod, J. C. 2006, VizieR Online Data Catalog, II/168

Miroshnichenko, A. S. 1996, A\&A, 312, 941

Miroshnichenko, A. S., Fremat, Y., Houziaux, L., et al. 1998, A\&AS, 131, 469

Morel, M., \& Magnenat, P. 1978, A\&AS, 34, 477

Muratorio, G., Rossi, C., \& Friedjung, M. 2008, A\&A, 487, 637

Najarro, F. 2001, in P Cygni 2000: 400 Years of Progress, eds. M. de Groot, \& C. Sterken, ASP Conf. Ser., 233, 133

Najarro, F., Hillier, D. J., \& Stahl, O. 1997, A\&A, 326, 1117

Ofek, E. O. 2008, PASP, 120, 1128

Okazaki, A. T., Owocki, S. P., Russell, C. M. P., \& Corcoran, M. F. 2008, MNRAS, 388, L39

Pastorello, A., Cappellaro, E., Inserra, C., et al. 2013, ApJ, 767, 1

Perryman, M. A. C., \& ESA 1997, The HIPPARCOS and TYCHO catalogues. Astrometric and photometric star catalogues derived from the ESA HIPPARCOS Space Astrometry Mission, ESA SP, 1200

Pojmanski, G. 2002, Acta Astron., 52, 397

Polidan, R. S. 1992, in IUE Proposal, 4281

Prša, A., \& Zwitter, T. 2005, ApJ, 628, 426

Raskin, G., van Winckel, H., Hensberge, H., et al. 2011, A\&A, 526, A69

Richardson, N. D., Gies, D. R., Henry, T. J., Fernández-Lajús, E., \& Okazaki, A. T. 2010, AJ, 139, 1534

Richardson, N. D., Gies, D. R., \& Williams, S. J. 2011, AJ, 142, 201

Richmond, M. W. 2007, PASP, 119, 1083

Ritchie, B. W., Clark, J. S., Negueruela, I., \& Najarro, F. 2009, A\&A, 507, 1597

Schaefer, G. H., Gies, D. R., Monnier, J. D., et al. 2010, AJ, 140, 1838

Smith, L. J., Crowther, P. A., \& Prinja, R. K. 1994, A\&A, 281, 833

Straizys, V., Kazlauskas, A., Jodinskiene, E., \& Bartkevicius, A. 1989, Bulletin d'Information du Centre de Données Stellaires, 37, 179

Swensson, J. W. 1943, ApJ, 97, 226

Tubbesing, S., Kaufer, A., Stahl, O., et al. 2002, A\&A, 389, 931

Wisniewski, J. P., Babler, B. L., Bjorkman, K. S., et al. 2006, PASP, 118, 820

Wolf, B., Kaufer, A., Rivinius, T., et al. 2000, in Thermal and Ionization Aspects of Flows from Hot Stars, eds. H. Lamers, \& A. Sapar, ASP Conf. Ser., 204, 43

Pages 21 to 28 are available in the electronic edition of the journal at http: //wWw . aanda. org 
A. Lobel et al.: Wind modelling of massive binary MWC 314

Table 1. High-resolution Mercator-Hermes (H) and ESO-Feros (F) échelle spectroscopic observations of MWC 314.

\begin{tabular}{lcccccccccc}
\hline \hline No. & Obs. date & HJD & Instr. & $\begin{array}{c}V_{r} \\
{\left[\mathrm{~km} \mathrm{~s}^{-1}\right]}\end{array}$ & $\begin{array}{c}\sigma\left(V_{r}\right) \\
{\left[\mathrm{km} \mathrm{s}^{-1}\right]}\end{array}$ & $\phi$ & $\begin{array}{c}\mathrm{H} \alpha \\
\text { Em. max. }\end{array}$ & $\begin{array}{c}\mathrm{H} \beta \\
\text { Em. max. }\end{array}$ & $\begin{array}{c}\text { Fe II } \lambda 6433 \\
\text { B/R max. }\end{array}$ & $\begin{array}{c}\text { Fe II } \lambda 6456 \\
\text { B/R max. }\end{array}$ \\
\hline 1 & $05-06-2009$ & 2454987.254 & $\mathrm{~F}$ & +62 & 7 & 0.440 & 24.969 & 6.602 & 0.934 & 0.960 \\
2 & $05-09-2009$ & 2455080.426 & $\mathrm{H}$ & +36 & 5 & 0.963 & 23.353 & 6.627 & 0.992 & 0.973 \\
3 & $10-09-2009$ & 2455084.459 & $\mathrm{H}$ & +69 & 4 & 0.039 & 24.206 & 6.589 & 0.967 & 0.926 \\
4 & $06-04-2010$ & 2455292.659 & $\mathrm{H}$ & +50 & 5 & 0.462 & 24.448 & 6.583 & 0.947 & 0.959 \\
5 & $29-06-2010$ & 2455376.635 & $\mathrm{H}$ & -70 & 5 & 0.795 & 24.462 & 7.171 & 1.090 & 1.011 \\
6 & $17-08-2010$ & 2455426.461 & $\mathrm{H}$ & -36 & 5 & 0.651 & 25.394 & 7.355 & 1.143 & 1.033 \\
7 & $27-09-2010$ & 2455467.383 & $\mathrm{H}$ & +82 & 4 & 0.325 & 22.843 & 6.392 & 0.885 & 0.910 \\
8 & $04-10-2010$ & 2455474.362 & $\mathrm{H}$ & +58 & 3 & 0.444 & 23.070 & 6.325 & 0.948 & 0.968 \\
9 & $29-10-2010$ & 2455499.325 & $\mathrm{H}$ & -44 & 3 & 0.852 & 23.706 & 6.647 & 1.039 & 0.992 \\
10 & $18-03-2011$ & 2455638.764 & $\mathrm{H}$ & +93 & 4 & 0.157 & 23.215 & 6.420 & 0.981 & 0.955 \\
11 & $21-03-2011$ & 2455641.764 & $\mathrm{H}$ & +99 & 6 & 0.208 & 23.421 & 6.392 & 0.942 & 0.939 \\
12 & $02-04-2011$ & 2455653.681 & $\mathrm{H}$ & +74 & 2 & 0.405 & 23.730 & 6.487 & 0.947 & 0.946 \\
13 & $26-05-2011$ & 2455708.691 & $\mathrm{H}$ & +89 & 6 & 0.310 & 22.974 & 6.196 & 0.873 & 0.864 \\
14 & $05-07-2011$ & 2455748.442 & $\mathrm{H}$ & +30 & 3 & 0.957 & 23.912 & 6.503 & 0.971 & 0.986 \\
15 & $27-07-2011$ & 2455769.542 & $\mathrm{H}$ & +85 & 2 & 0.318 & 23.667 & 6.442 & 0.894 & 0.901 \\
16 & $17-08-2011$ & 2455791.546 & $\mathrm{H}$ & -38 & 3 & 0.656 & 25.621 & 7.127 & 1.105 & 1.023 \\
\hline
\end{tabular}

Notes. The radial velocity $V_{r}$-value is given in the heliocentric rest frame. $\phi$ denotes the orbital phase. Em. max. is the continuum normalised line flux maximum. B/R max. denotes the ratio of blue and red normalised maximum emission fluxes of double-peaked lines.

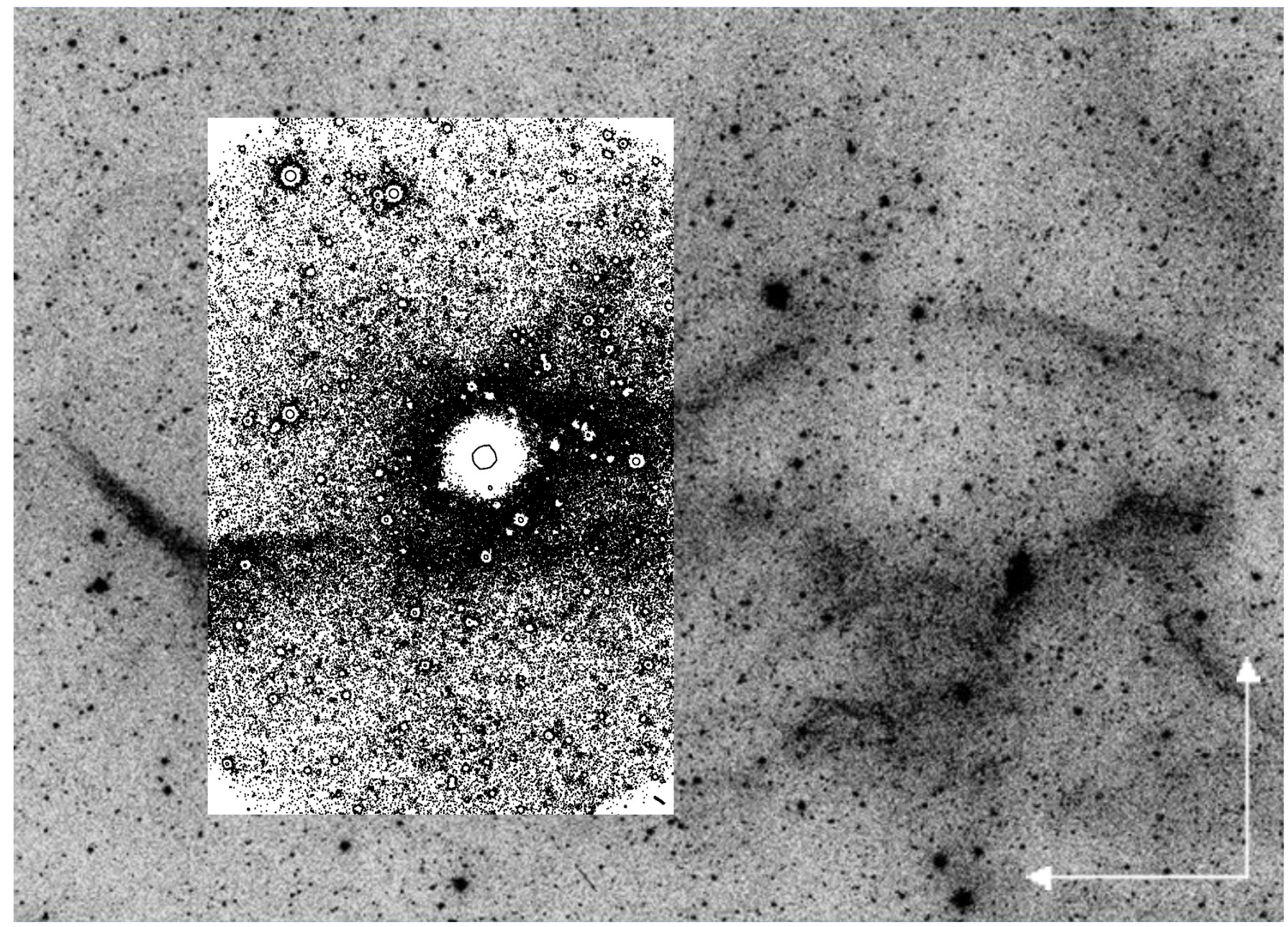

Fig. 1. Narrow-band $\mathrm{H} \alpha$ image of the extended bipolar nebula of MWC 314 (adapted from Marston \& McCollum 2008). The image is 12.5' vertically. The inset shows the Mercator-MEROPE intensity contour image with faint $\mathrm{H} \alpha$ nebulosity surrounding the central star. North is up, and east to the left. 

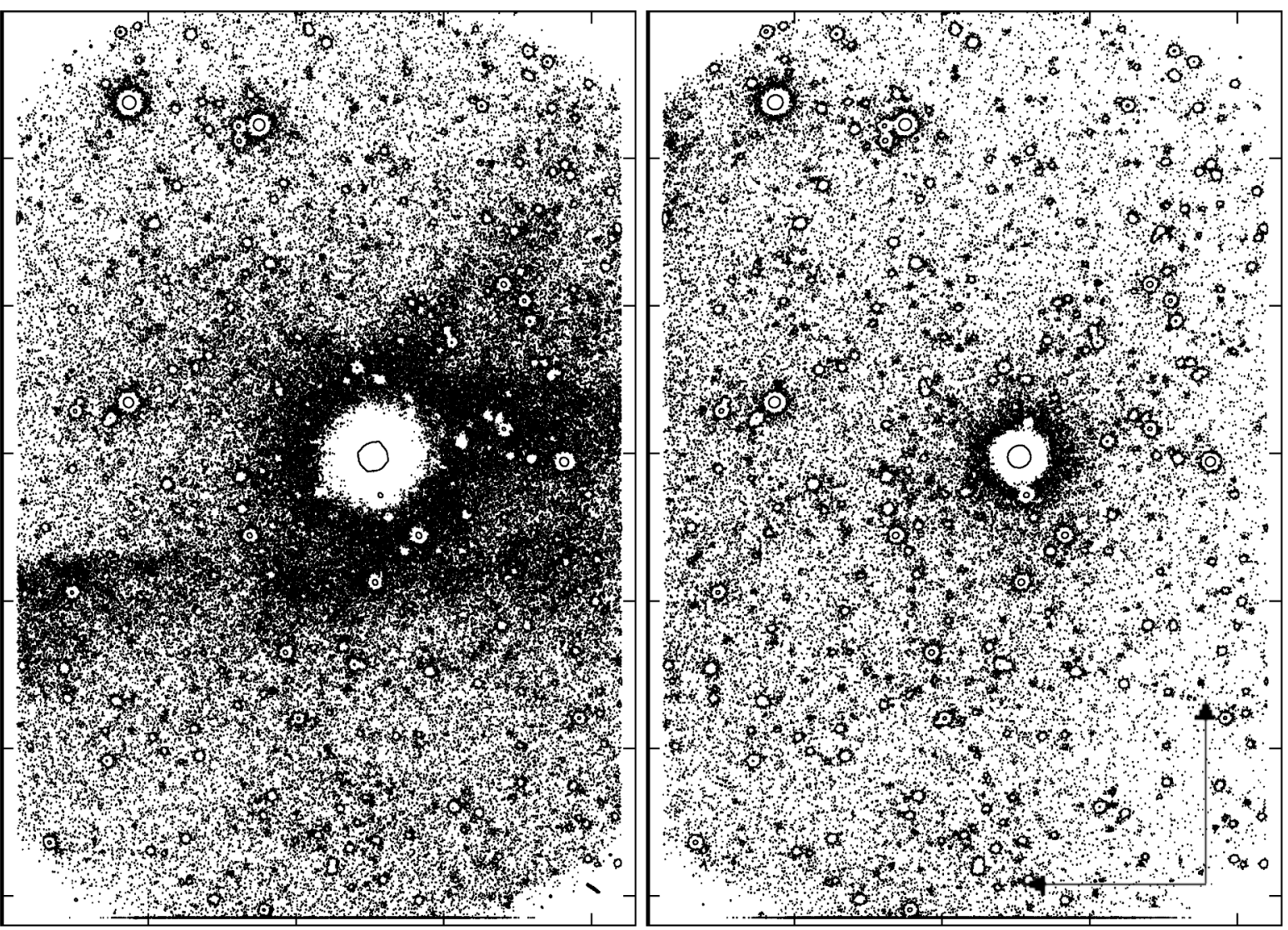

Fig. 2. Comparison of the (intensity contour) narrow-band $\mathrm{H} \alpha$ emission image (left-hand panel) and the continuum image (right-hand panel) of MWC 314 observed in March 2011 with Mercator-MEROPE. Line emission is absent in the continuum image where extended east-west filament structures are observed in the $\mathrm{H} \alpha$ emission image (see Sect. 3). Both panels are 6.9' by $10^{\prime}$. North is up, and east to the left.

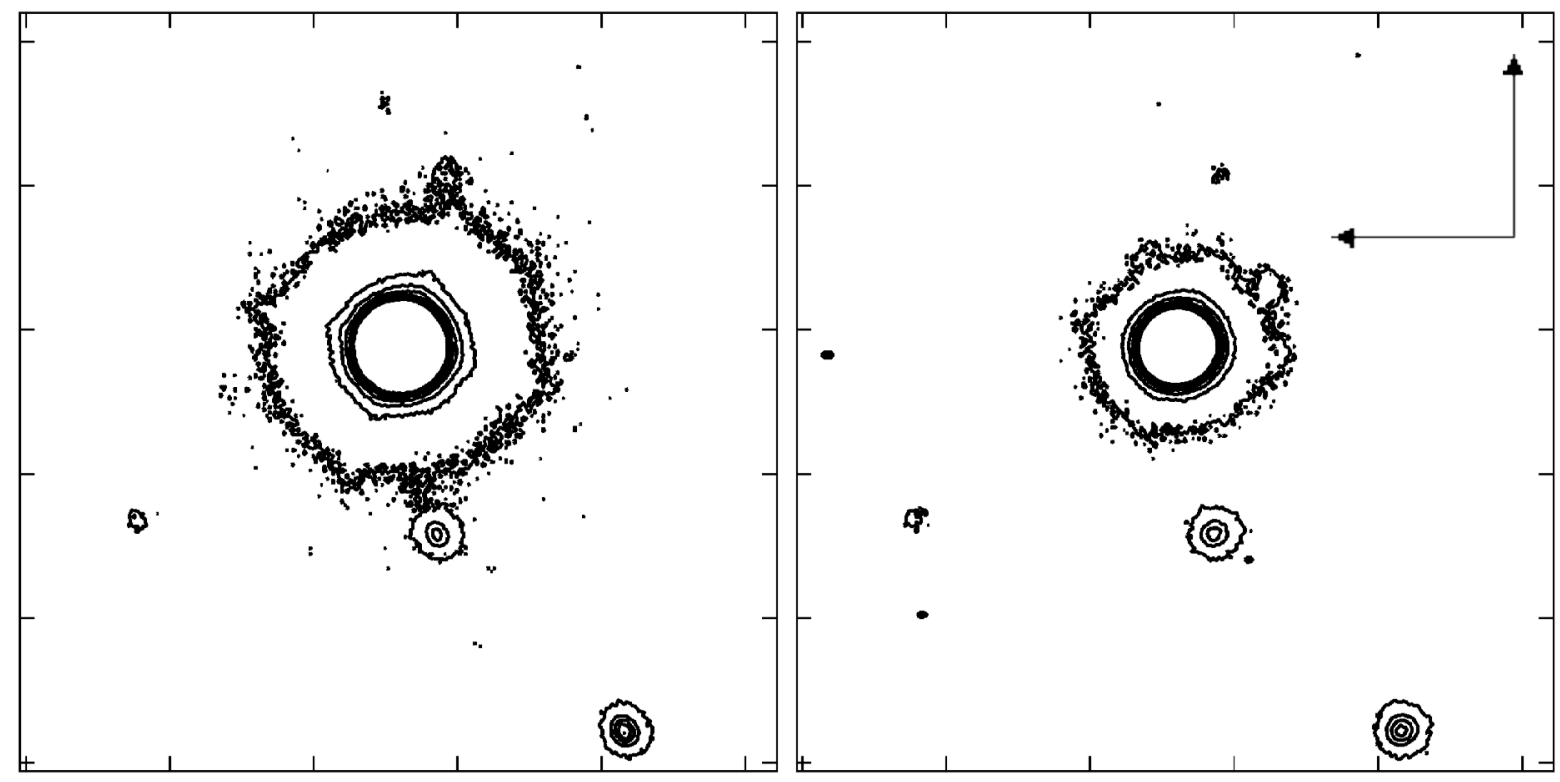

Fig. 3. Comparison of $100^{\prime \prime} \times 100^{\prime \prime} \mathrm{H} \alpha$ (left-hand panel) and continuum (right-hand panel) intensity contour images of MWC 314. The outer intensity contour signals an extended $\mathrm{H} \alpha$ envelope that is almost circular symmetric within $20^{\prime \prime}$ of the central star. 


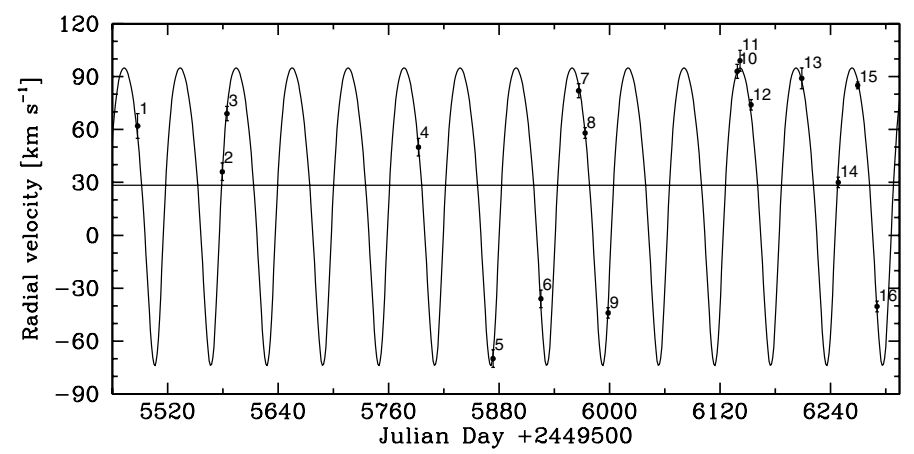

Fig. 4. Best fit orbital solution to 16 radial velocity measurements in MWC 314. The RV observation dates during $22 \mathrm{~m}$ are given in Table 1. The solid horizontal line is drawn at the $\gamma$-velocity for the best fit orbital solution.


Fig. 9. Top panels: dynamic spectra of three absorption lines of MWC 314 in the heliocentric velocity scale. Blue colours correspond to normalised stellar continuum flux levels. Bottom panels: corresponding normalised line profiles shifted upwards with orbital phase (spectrum numbers are labelled in the left-hand panel). The (S-shaped) Doppler shifts of the lines is due to the orbital motion of the primary star. We observe significant enhancements in the absorption line depth at orbital phases with large Doppler blue-shift of $\phi=0.65-0.85$. 
A\&A 559, A16 (2013)
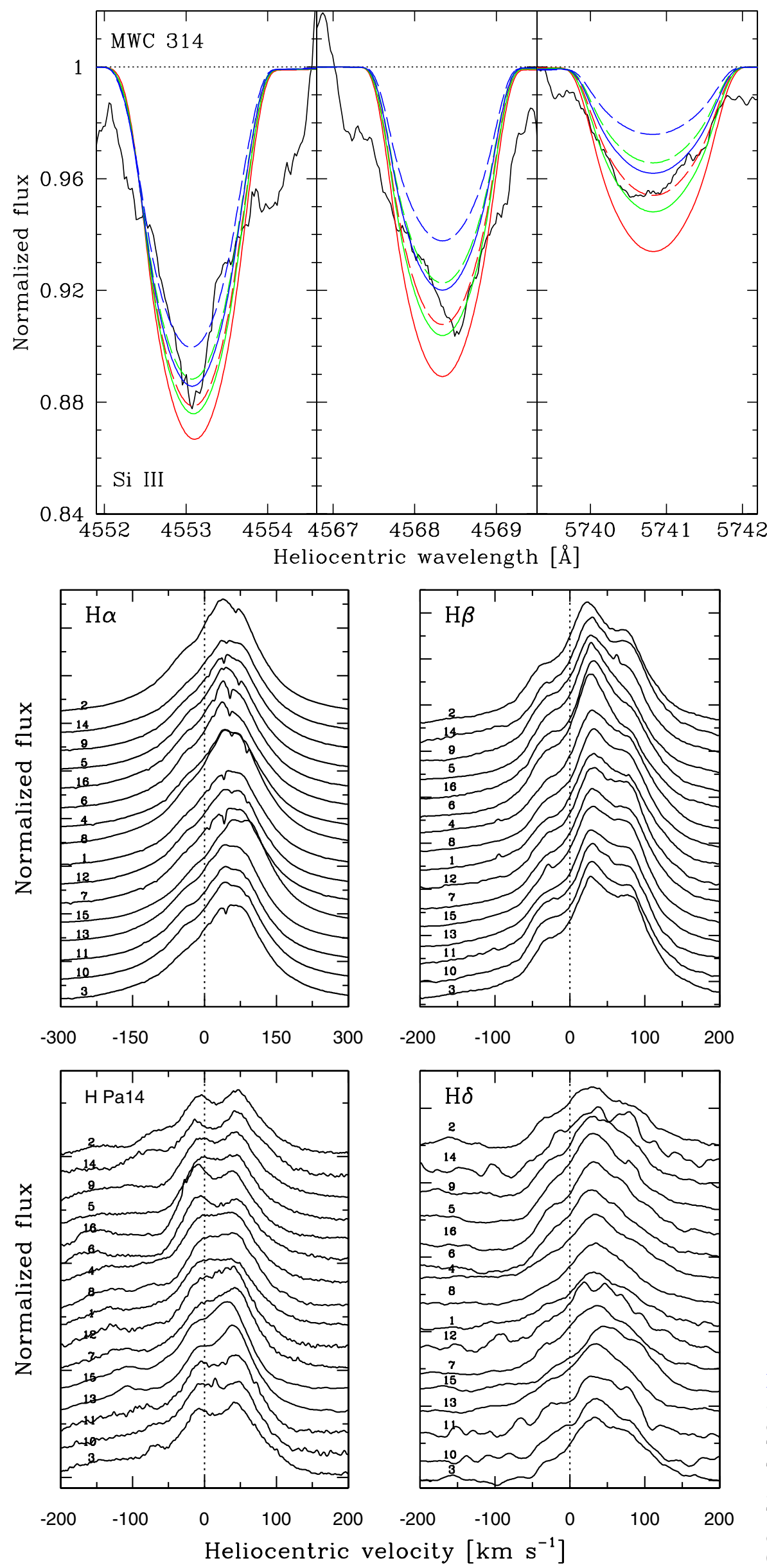

Fig. 10. Three Si III absorption lines observed in MWC 314 (black solid lines) are overplotted with theoretical line profiles computed in nonLTE with Multi. The line transfer calculations use atmosphere models of $T_{\text {eff }}=17 \mathrm{kK}$ (dashed drawn lines), $18 \mathrm{kK}$ (solid drawn lines), and 19 kK (dash-dotted lines). Boldly drawn lines are computed with $\log g=2.5$, and thin drawn lines for $\log g=3.0$. Atmosphere models with $T_{\text {eff }}>$ $19 \mathrm{kK}$ yield Si III line equivalent widths incompatible with observed values. The detailed profile fits require $V_{\text {rot }} \sin i \simeq 50 \mathrm{~km} \mathrm{~s}^{-1}$.
Fig. 14. Normalised $\mathrm{H} \alpha, \mathrm{H} \beta, \mathrm{H} \delta$, and $\mathrm{H} \mathrm{Pa} 14$ lines are plotted with $\phi$ (clockwise). The line FWHM decreases towards the higher $\mathrm{H}$ Balmer series lines. The $\mathrm{H} \alpha$ line wings extend beyond $\pm 300 \mathrm{~km} \mathrm{~s}^{-1}$. The higher Balmer series lines also show stronger absorption in the violet line wing to $\sim 300 \mathrm{~km} \mathrm{~s}^{-1}$. The $\mathrm{H} \delta$ and $\mathrm{Pa} 14$ lines (bottom panels) show enhanced blue-shifted absorption around $\phi=$ $0.65-0.85$, similar to the He I lines. 

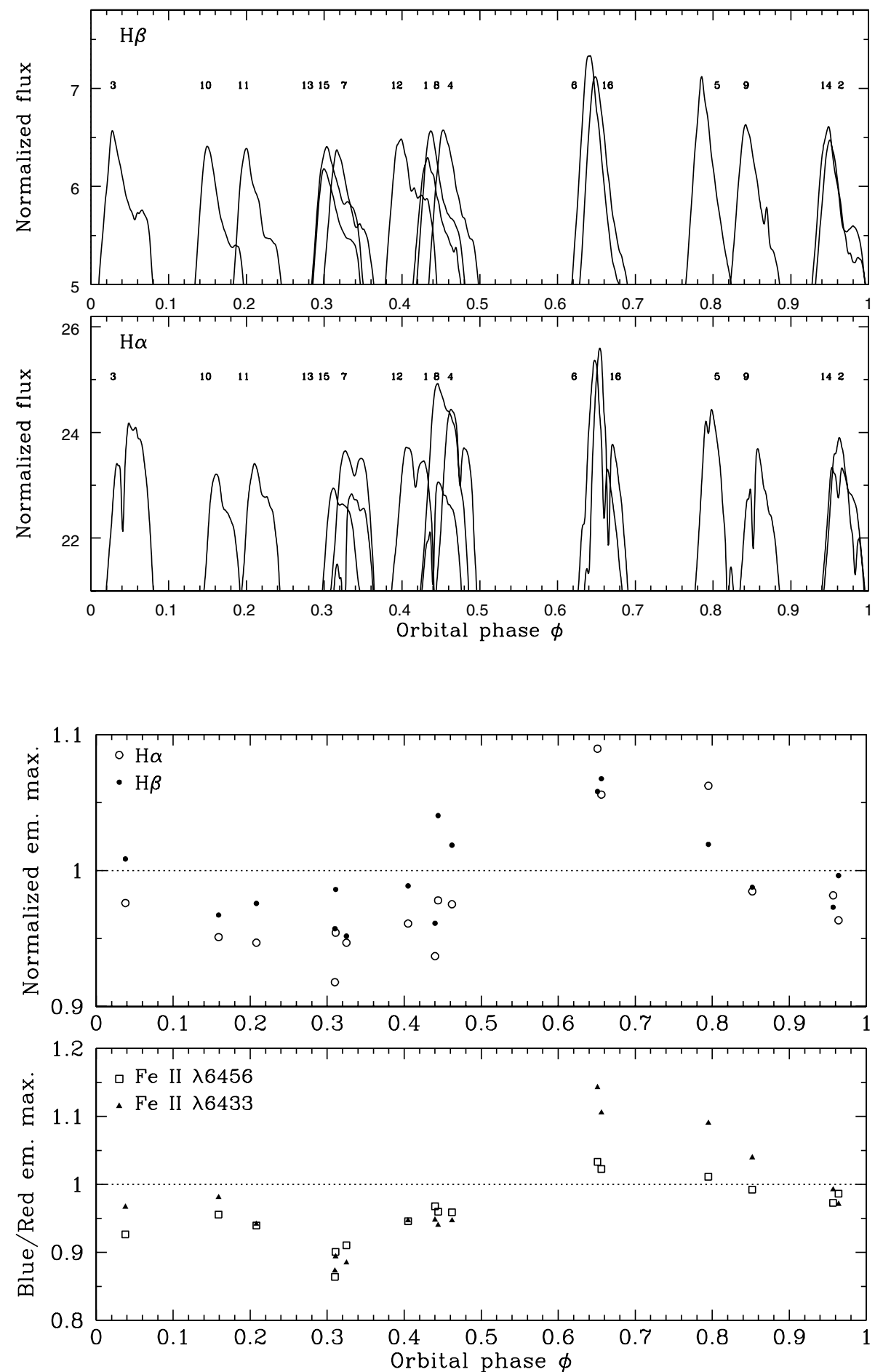

Fig. 15. $\mathrm{H} \alpha$ (bottom panel) and $\mathrm{H} \beta$ (top panel) emission lines of MWC 314 show largest normalised flux maxima at $\phi \simeq 0.65$ (spectrum Nos. 6 and 16), and smallest flux maxima at $\phi \simeq 0.32$ (Nos. 13, 15, and 7). The absolute emission line fluxes determined with the $V$ brightness curve are however almost invariable, indicating that the extended circumbinary $\mathrm{H} \alpha$ and $\mathrm{H} \beta$ emission line formation regions are little influenced by the orbital motion.

Fig. 16. Top panel: continuum normalised flux maxima observed with $\phi$ in strong $\mathrm{H} \alpha$ (open symbols) and $\mathrm{H} \beta$ (dots) emission lines of MWC 314 . The $\mathrm{H} \alpha$ maxima are divided by 24 , and $\mathrm{H} \beta$ maxima by 6.75 for comparison. Bottom panel: blue/red emission peak ratio we observe in two permitted Fe II emission lines formed in the circumbinary disc. The $\mathrm{B} / \mathrm{R}$ variations of $\pm 10 \%$ are due to orbital modulation of Doppler shifting line opacity, whereas the $\mathrm{H} \alpha$ and $\mathrm{H} \beta$ flux changes result from variability of the stellar continuum flux with $\phi$. 

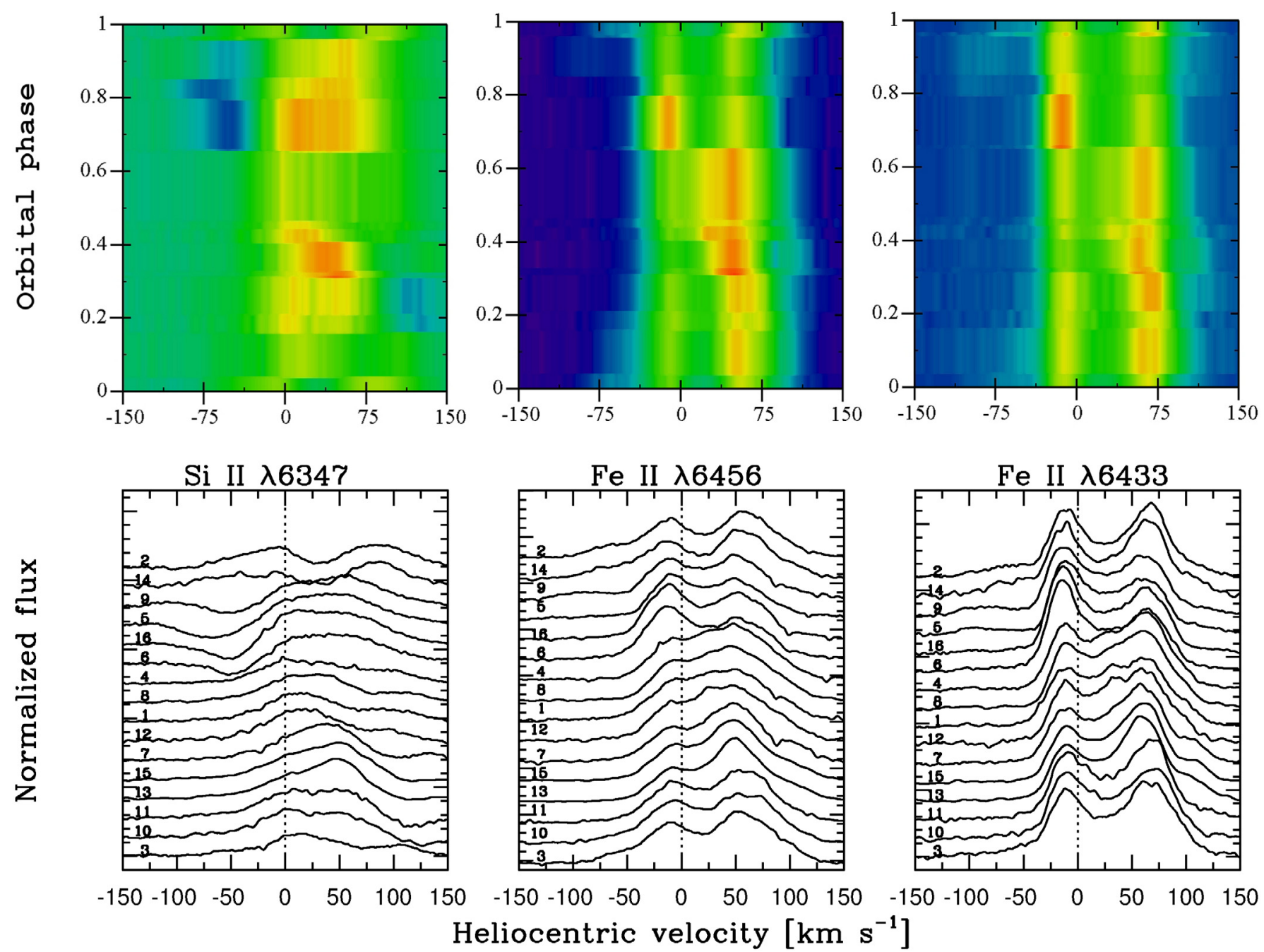

Fig. 18. Detailed profiles of permitted Fe II lines (middle and right-hand panels) strongly depend on $\phi$. The prominent emission lines form in a circumbinary disc and stay static (within $\pm 3 \mathrm{~km} \mathrm{~s}^{-1}$ ) around the $\gamma$-velocity. The B/R emission peak ratios (see bottom panel of Fig. 14) periodically vary due to Doppler shifting lines formed in the orbiting primary star. It causes the triple-peaked emission lines around $\phi=0.4-0.5$. We observe in Si II $\lambda 6347$ static line emission from the disc modulated by Doppler shifting absorption in the primary (left-hand panels). 

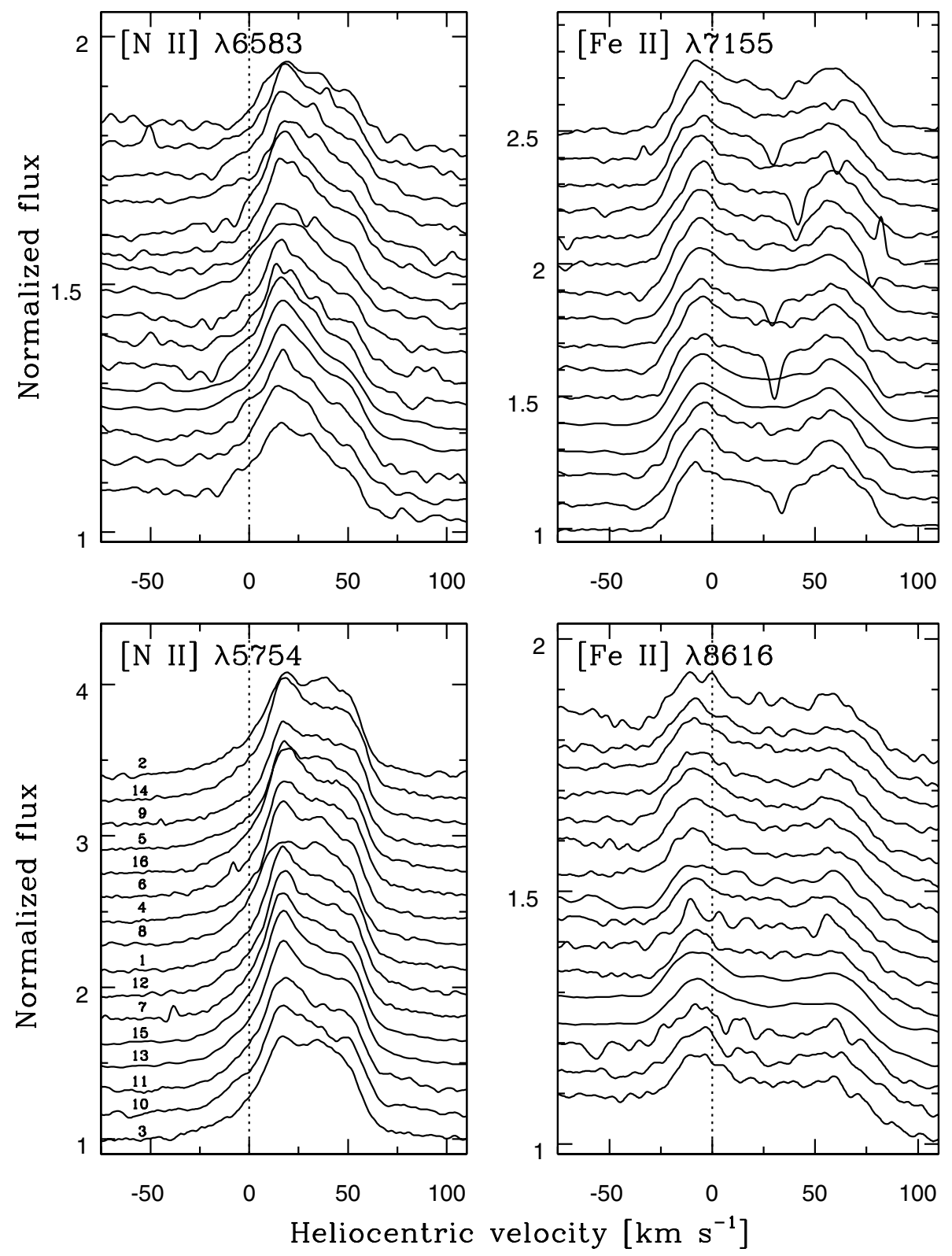

Fig. 19. Normalised fluxes plotted with $\phi$ (shifted upwards) of two forbidden [N II] (lefthand panels) and two [Fe II] (right-hand panels) emission lines in MWC 314. The FWHM $\simeq$ $50 \mathrm{~km} \mathrm{~s}^{-1}$ of the double-peaked [Fe II] emission lines is comparable to the permitted Fe II emission lines (see Fig. 16), indicating a common line formation region in a circumbinary disc. The $[\mathrm{N}$ II] lines show smaller $F W H M \simeq$ $25 \mathrm{~km} \mathrm{~s}^{-1}$.

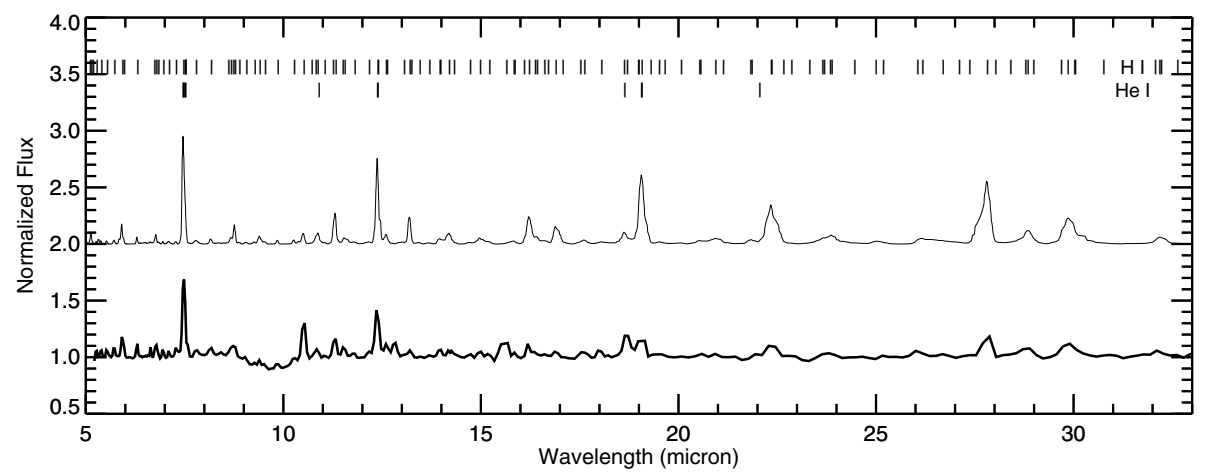

Fig. 20. Comparison of the Spitzer spectrum of MWC 314 (bottom spectrum) and the theoretical model spectrum (top spectrum). The vertical lines mark the wavelength positions of $\mathrm{H} \mathrm{I}$ and $\mathrm{He}$ II lines used in the spectrum calculations. The prominent emission lines computed at $22.38 \mu \mathrm{m}$ and $27.8 \mu \mathrm{m}$ are due to $\mathrm{H}$ I and He I lines (see text). 

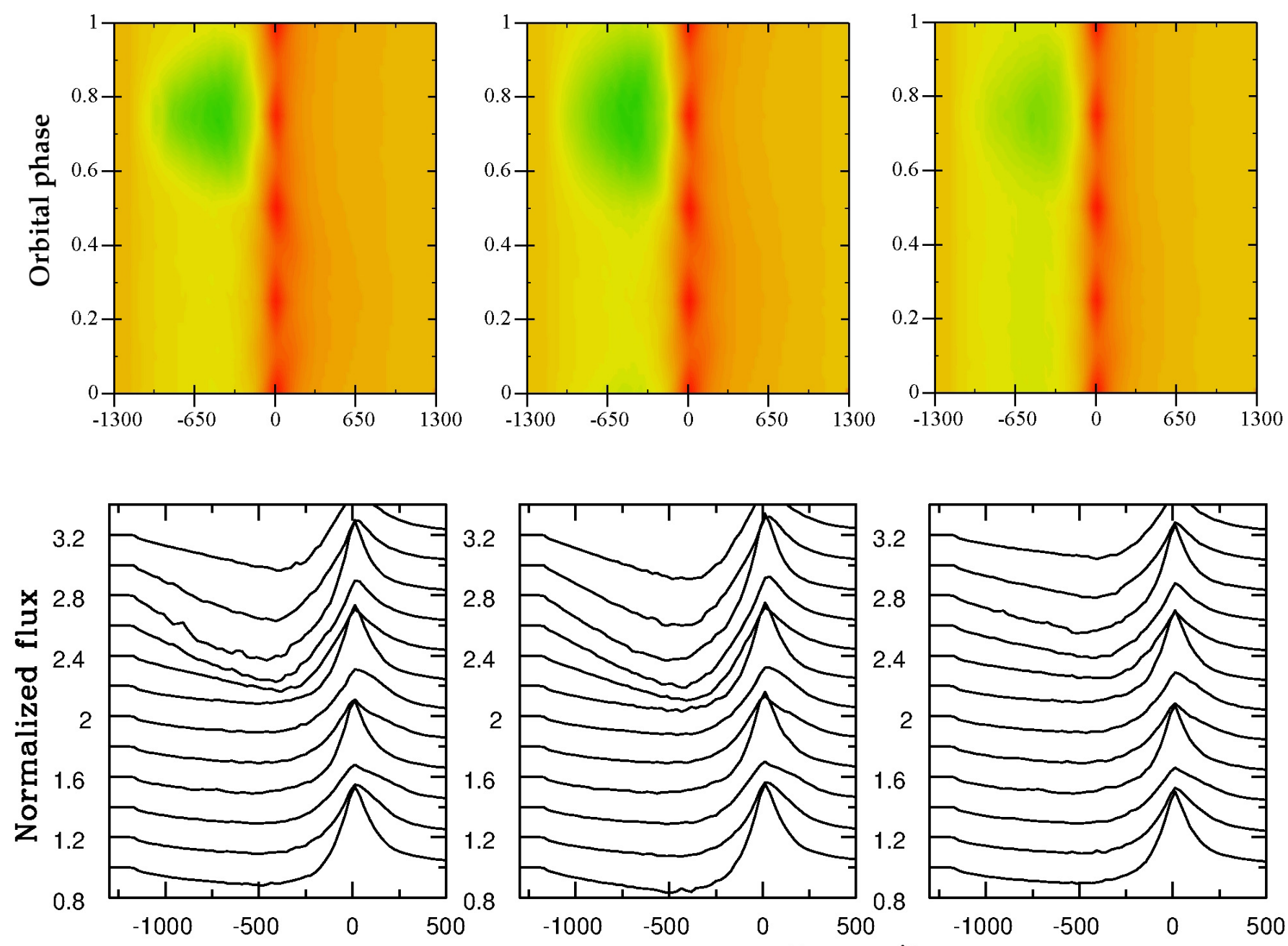

Stellar rest velocity $\left[\mathrm{km} \mathrm{s}^{-1}\right]$

Fig. 23. Top panels: dynamic spectra with orbital phase $\phi$ of He I $\lambda 5876$ computed with the 3D asymmetric wind model we develop for MWC 314 in this paper. Bottom panels: corresponding normalised flux spectra for $12 \phi$-values. Left-hand panels are computed for $f=10$ and $\varsigma_{0}=\vartheta_{0}=\pi / 4$ in the density structure of Eq. (6). For comparison the middle panels are computed for $f=10, \varsigma_{0}=\vartheta_{0}=\pi / 3$, with the radial velocity amplitude of orbital motion set to $4 \times$ the observed value. Right-hand panel is computed for $f=3.3, \varsigma_{0}=\vartheta_{0}=\pi / 4$, and the observed $V_{r}$-curve. The latter values provide the best fit to the P Cyg absorption line profile changes we observe with $\phi$ shown in Fig. 12 (see Sect. 5.3). 